\title{
Averages of central $L$-values of Hilbert modular forms with an application to subconvexity
}

\author{
Brooke Feigon* \\ Institute for Advanced Study \\ Einstein Drive \\ Princeton, NJ 08450
}

\author{
David Whitehouse ${ }^{\dagger}$ \\ Institute for Advanced Study \\ Einstein Drive \\ Princeton, NJ 08450
}

\begin{abstract}
We use the relative trace formula to obtain exact formulas for central values of certain twisted quadratic base change $L$-functions averaged over Hilbert modular forms of a fixed weight and level. We apply these formulas to the subconvexity problem for these $L$-functions. We also establish an equidistribution result for the Hecke eigenvalues weighted by these $L$ values.
\end{abstract}

\section{Contents}

1 Introduction 2

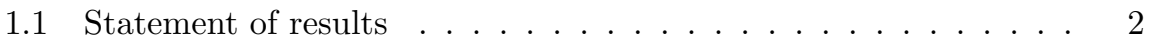

1.2 About the proof $\ldots \ldots \ldots \ldots \ldots \ldots \ldots$

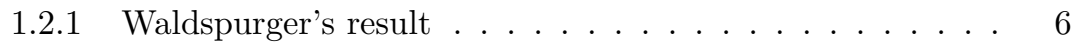

1.2 .2 Relative trace formula . . . . . . . . . . . . . 6

1.2.3 The case of modular forms of weight $2 \ldots \ldots \ldots$

1.3 About the paper . . . . . . . . . . . . . . 10

1.3.1 Summary of conditions . . . . . . . . . . . . 10

1.3 .2 Outline of the paper . . . . . . . . . . . . . 11

1.4 Acknowledgements . . . . . . . . . . . . . . . . 11

2 Notation 12

2.1 Normalization of measures . . . . . . . . . . . . . . 13

3 Spectral side of the trace formula $\quad 14$

3.1 The test function . . . . . . . . . . . . . . . 16

3.2 Local preliminaries . . . . . . . . . . . . . . . . . . 16

3.3 Global calculations . . . . . . . . . . . . . . . . . . . 18

*Email address: bfeigon@math.toronto.edu

${ }^{\dagger}$ Email address: dw@math.mit.edu 
4 Geometric side of the trace formula 22

4.1 Irregular cosets . . . . . . . . . . . . . . . . . 23

4.2 Regular cosets . . . . . . . . . . . . . . . . . . . . 28

4.2 .1 Exact calculations . . . . . . . . . . . . . . . . 29

4.2 .2 Bounds on $I\left(\xi, \mathbf{1}_{v}\right) \ldots \ldots \ldots \ldots$

4.3 Global calculations . . . . . . . . . . . . . . . . . . . . 35

5 A measure on the Hecke algebra 39

5.1 An application of the Plancherel formula . . . . . . . . . . . 39

5.2 The distribution $\tilde{I} \ldots \ldots \ldots \ldots \ldots \ldots$

6 Main results 42

6.1 Average $L$-values . . . . . . . . . . . . . . . . . . . . . . . . . . 43

6.2 Subconvexity . . . . . . . . . . . . . . . . . . 46

6.3 Classical reformulation . . . . . . . . . . . . . . . . 53

\section{Introduction}

\subsection{Statement of results}

In this paper we use the relative trace formula, together with period formulas originating in work of Waldspurger [Wal85], to study central values of $L$ functions associated to Hilbert modular forms. Let $F$ be a totally real number field and let $\Sigma_{\infty}$ denote the set of archimedean places of $F$. Given an ideal $\mathfrak{N}$ of $\mathcal{O}_{F}$ and a tuple of positive integers $\boldsymbol{k}=\left(k_{v}: v \in \Sigma_{\infty}\right)$ we let $\mathcal{F}(\mathfrak{N}, 2 \boldsymbol{k})$ denote the set of cuspidal automorphic representations of $\operatorname{PGL}\left(2, \mathbf{A}_{F}\right)$ which are of exact level $\mathfrak{N}$ and holomorphic of weight $2 \boldsymbol{k}$. We recall that each $\pi \in \mathcal{F}(\mathfrak{N}, 2 \boldsymbol{k})$ may be identified with a normalized holomorphic Hilbert modular newform of level $\mathfrak{N}$, weight $2 \boldsymbol{k}$ and trivial nebentypus which is an eigenfunction for all the Hecke operators.

Let $E$ be a quadratic extension of $F$ and for each $\pi \in \mathcal{F}(\mathfrak{N}, 2 \boldsymbol{k})$ let $\pi_{E}$ denote the base change of $\pi$ to an automorphic representation of $\operatorname{PGL}\left(2, \mathbf{A}_{E}\right)$. Given a unitary character $\Omega$ of the idele class group $E^{\times} \backslash \mathbf{A}_{E}^{\times}$of $E$ one may consider the completed $L$-function $L\left(s, \pi_{E} \otimes \Omega\right)$ which satisfies a functional equation relating $s$ to $1-s$. We note that if $\sigma_{\Omega}$ denotes the induction of $\Omega$ to an automorphic representation of $\mathrm{GL}\left(2, \mathbf{A}_{F}\right)$ then,

$$
L\left(s, \pi_{E} \otimes \Omega\right)=L\left(s, \pi \times \sigma_{\Omega}\right) .
$$

The object of study in this paper is $L\left(1 / 2, \pi_{E} \otimes \Omega\right)$, the central value of this $L$-function. In particular we prove an explicit formula for $L\left(1 / 2, \pi_{E} \otimes \Omega\right)$ as one averages over $\pi$ of a fixed weight and level. As an application of this formula we establish subconvexity as $\pi$ and $\Omega$ vary in a certain range and prove an equidistribution result for the Hecke eigenvalues of such $\pi$.

Throughout this paper we make the following assumptions on the data introduced above. 
- $E$ is an imaginary quadratic extension of $F$,

- $\mathfrak{N}$ is squarefree, each prime $\mathfrak{p}$ dividing $\mathfrak{N}$ is inert and unramified in $E$ and the number of primes dividing $\mathfrak{N}$ has the same parity as the degree of the extension $[F: \mathbf{Q}]$, and

- the character $\Omega$ is trivial when restricted to $\mathbf{A}_{F}^{\times}$, unramified at the places of $E$ above $\mathfrak{N}$, and at each archimedean place $\Omega_{v}$ has weight $m_{v}<k_{v}$.

See Section 1.3.1 below for a discussion on the relevance and seriousness of these assumptions.

The results of this paper are all derived from an exact formula for

$$
\sum_{\pi \in \mathcal{F}(\mathfrak{N}, 2 k)} \frac{L\left(1 / 2, \pi_{E} \otimes \Omega\right)}{L(1, \pi, A d)} \hat{f}_{\mathfrak{p}}\left(\pi_{\mathfrak{p}}\right)
$$

obtained via the relative trace formula. Here $L(s, \pi, A d)$ is the adjoint $L$ function of $\pi$ and $\hat{f}_{\mathfrak{p}}$ denotes a Hecke operator at $\mathfrak{p} \nmid \mathfrak{N}$.

When $\mathfrak{N}$ is large with respect to $E, c(\Omega)$ (the conductor of $\Omega$ ) and $f_{\mathfrak{p}}$ our formula simplifies considerably. The simplest version of it is given by,

Theorem 1.1. Assume that not all $k_{v}=1$ and $\mathfrak{N}$ has absolute norm larger than $d_{E / F} c(\Omega)^{h_{F}}$. Then,

$$
\frac{2^{[F: \mathbf{Q}]}}{|\mathfrak{N}|}\left(\begin{array}{c}
2 \boldsymbol{k}-2 \\
\boldsymbol{k}+\boldsymbol{m}-1
\end{array}\right) \sum_{\pi \in \mathcal{F}(\mathfrak{N}, 2 \boldsymbol{k})} \frac{L\left(1 / 2, \pi_{E} \otimes \Omega\right)}{L(1, \pi, A d)}=4\left|\Delta_{F}\right|^{\frac{3}{2}} L^{S(\Omega)}(1, \eta)
$$

where $S(\Omega)$ denotes the set of places of $F$ above which $\Omega$ is ramified and $\eta$ is the quadratic idele class character of $F$ associated to $E$.

For the full formula see Section 6 and for any undefined notation see Section 2 .

Over $\mathbf{Q}$ we can express this formula more classically. We identify $\mathcal{F}(N, 2 k)$ with the set of normalized modular newforms of level $N$ and weight $2 k$ which are Hecke eigenforms. Let $E=\mathbf{Q}(\sqrt{-d})$ be an imaginary quadratic extension of $\mathbf{Q}$ of discriminant $-d$. We take $\Omega$ as before and let $g_{\Omega}$ denote the modular form of weight $2|m|+1$, level $d c(\Omega)$ and nebentypus $\chi_{-d}$ associated to $\Omega$. For $f \in \mathcal{F}(N, 2 k)$ we take the Rankin-Selberg $L$-function, $L\left(s, f \times g_{\Omega}\right)$ which has functional equation relating $s$ to $2 k+2|m|+1-s$. Using the relationship between $L\left(1, \pi_{f}, A d\right)$, where $\pi_{f}$ denotes the automorphic representation generated by $f$, and the square of the Petersson norm of $f$,

$$
(f, f)=\int_{\Gamma_{0}(N) \backslash \mathcal{H}}|f(x+i y)| y^{2 k} \frac{d x d y}{y^{2}}
$$

we can rewrite Theorem 1.1 in the following way: Assume $\Omega$ is not quadratic, $k>1$ and $N>d c(\Omega)$, then

$$
\frac{(2 k-2) ! u_{-d} \sqrt{d} L_{S(\Omega)}\left(1, \chi_{-d}\right)}{2 \pi(4 \pi)^{2 k-1}} \sum_{f \in \mathcal{F}(N, 2 k)} \frac{L_{f i n}\left(k+|m|+\frac{1}{2}, f \times g_{\Omega}\right)}{(f, f)}=h_{-d}
$$


where $\chi_{-d}$ is the quadratic Dirichlet character of discriminant $-d, h_{-d}$ is the class number of $E$ and $u_{-d}=\# \mathcal{O}_{E}^{\times} /\{ \pm 1\}$. See Section 6.3 for further details.

We note that over $\mathbf{Q}$ and with $\Omega$ trivial an asymptotic version of Theorem 1.1 has been known for a while. First by Duke [Duk95] for prime level and weight 2 and then by Iwaniec, Luo and Sarnak [IS00b], [ILS00] for squarefree level and higher weight. An exact formula has been established by Michel and Ramakrishnan in the case $F=\mathbf{Q}$ and $\Omega$ is a character of the ideal class group of $E$. Their work uses Gross' formula together with a geometric argument in the weight two case, and the theta correspondence in higher weight; see [MR].

By an extension of Theorem 1.1 to include Hecke operators, we obtain a result that includes a restriction at a prime $\mathfrak{p} \nmid \mathfrak{N}$. Let $\pi=\otimes_{v} \pi_{v} \in \mathcal{F}(\mathfrak{N}, 2 \boldsymbol{k})$. We let $\left\{\alpha_{\mathfrak{p}}, \alpha_{\mathfrak{p}}^{-1}\right\}$ denote the Satake parameters of $\pi_{\mathfrak{p}}$ and set $a_{\mathfrak{p}}(\pi)=\alpha_{\mathfrak{p}}+$ $\alpha_{\mathfrak{p}}^{-1}$. We recall that $a_{\mathfrak{p}}(\pi) \in[-2,+2]$ by Ramanujan's conjecture; [Bla06]. The distribution of the $a_{\mathfrak{p}}(\pi)$ has been considered by Sarnak [Sar87] and Serre [Ser97]. The spherical Plancherel measure on $\operatorname{PGL}\left(2, F_{\mathfrak{p}}\right)$ is given by

$$
\mu_{\mathfrak{p}}=\frac{q_{\mathfrak{p}}+1}{\left(q_{\mathfrak{p}}^{\frac{1}{2}}+q_{\mathfrak{p}}^{-\frac{1}{2}}\right)^{2}-x^{2}} \frac{\sqrt{4-x^{2}}}{2 \pi} d x
$$

on $[-2,2]$, here $q_{\mathfrak{p}}$ denotes the order of the residue field at $\mathfrak{p}$. Serre has proven that when $F=\mathbf{Q}$,

$$
\left\{a_{\mathfrak{p}}(\pi): \pi \in \mathcal{F}(\mathfrak{N}, 2 \boldsymbol{k})\right\}
$$

become equidistributed with respect to $\mu_{\mathfrak{p}}$ as $\mathfrak{N} \rightarrow \infty$. We prove a variant of this result where we include a weighting by $L^{\mathfrak{p}}\left(1 / 2, \pi_{E} \otimes \Omega\right)$.

Theorem 1.2. For any $J \subset[-2,+2]$ we have

$$
\begin{aligned}
& \lim _{\mathfrak{N} \rightarrow \infty} \frac{1}{|\mathfrak{N}|} \sum_{\substack{\pi \in \mathcal{F}(\mathfrak{N}, 2 k) \\
a_{\mathfrak{p}}(\pi) \in J}} \frac{L^{\mathfrak{p}}\left(1 / 2, \pi_{E} \otimes \Omega\right)}{L^{\mathfrak{p}}(1, \pi, A d)} \\
= & 4\left|\Delta_{F}\right|^{\frac{3}{2}} \frac{1}{2^{[F: \mathbf{Q}]}}\left(\begin{array}{c}
2 \boldsymbol{k}-2 \\
\boldsymbol{k}+\boldsymbol{m}-1
\end{array}\right)^{-1} L^{S(\Omega) \cup\{\mathfrak{p}\}}(1, \eta) L\left(2,1_{F_{\mathfrak{p}}}\right) \mu_{\mathfrak{p}}(J) .
\end{aligned}
$$

We note that in the case that $F=\mathbf{Q}$ and $\Omega$ is trivial, we recover the main result of [RR05]. A similar result has been obtained by Royer [Roy00] for the single $L$-value $L(1 / 2, \pi)$ averaged over modular forms $\pi$ of level $N$ and weight 2 .

We remark that one could consider the average by normalizing by $|\mathcal{F}(\mathfrak{N}, 2 \boldsymbol{k})|$ rather than $|\mathfrak{N}|$. In order to have a finite limit with this normalization we need to add the technical restriction thatb $|\mathfrak{N}| \prod_{\mathfrak{p} \mid \mathfrak{N}}\left(1-\frac{1}{|\mathfrak{p}|}\right) \sim|\mathfrak{N}|$. For $F=\mathbf{Q}$ this condition reduces to $\varphi(N) \sim N$ where $\varphi$ is the Euler totient function. Using the well known fact that $|\mathcal{F}(N, 2 k)| \sim \frac{2 k-1}{12} \varphi(N)$ as $N \rightarrow \infty$ we get the following statement. 
Corollary 1.3. Let $F=\mathbf{Q}$ and $J \subset[-2,+2]$. Then

$$
\lim _{N \rightarrow \infty} \frac{1}{|\mathcal{F}(N, 2 k)|} \sum_{\substack{\pi \in \mathcal{F}(N, 2 k) \\ a_{p}(\pi) \in J}} \frac{L^{p}\left(1 / 2, \pi_{E} \otimes \Omega\right)}{L^{p}(1, \pi, A d)}
$$

is equal to

$$
\frac{24}{2 k-1}\left(\begin{array}{c}
2 k-2 \\
k+m-1
\end{array}\right)^{-1} L^{S(\Omega) \cup\{p\}}(1, \eta) L\left(2,1_{\mathbf{Q}_{p}}\right) \mu_{p}(J)
$$

where the limit is taken over squarefree $N$ such that $\varphi(N) \sim N$ and each prime dividing $N$ is inert and unramified in $E$ and does not divide $c(\Omega)$.

Finally we apply our work to the problem of subconvexity. Using a version of Theorem 1.1 that is also valid for smaller $\mathfrak{N}$, combined with the non-negativity of $L\left(1 / 2, \pi \times \sigma_{\Omega}\right)$, established in [JC01], and an upper bound for $L(1, \pi, A d)$, we get the following theorem.

Theorem 1.4. Fix a totally real number field $F$ and a $C M$ extension $E$ of $F$. Let $\mathfrak{N}$ be a squarefree ideal in $\mathcal{O}_{F}$ such that the number of primes dividing $\mathfrak{N}$ has the same parity as $[F: \mathbf{Q}]$ and such that each prime of $F$ dividing $\mathfrak{N}$ is inert and unramified in $E$. Let $\Omega$ be a character of $\mathbf{A}_{F}^{\times} E^{\times} \backslash \mathbf{A}_{E}^{\times}$which is unramified above $\mathfrak{N}$ and has weights at the archimedean places strictly less than $\boldsymbol{k}$. Then for any $\epsilon>0$,

$$
L_{f i n}\left(1 / 2, \pi \times \sigma_{\Omega}\right) \ll_{F, E, \boldsymbol{k}, \epsilon}|\mathfrak{N}|^{1+\epsilon} c(\Omega)^{\epsilon}+|\mathfrak{N}|^{\epsilon} c(\Omega)^{\frac{1}{2}+\epsilon},
$$

for all $\pi \in \mathcal{F}(\mathfrak{N}, 2 \boldsymbol{k})$.

Hence for $0 \leq t<\frac{1}{6}$ and $\epsilon>0$,

$$
L_{f i n}\left(1 / 2, \pi \times \sigma_{\Omega}\right) \ll_{F, E, \boldsymbol{k}, \epsilon}(c(\Omega)|\mathfrak{N}|)^{\frac{1}{2}-t},
$$

for $\pi \in \mathcal{F}(\mathfrak{N}, 2 \boldsymbol{k})$ with $\mathfrak{N}$ such that

$$
c(\Omega)^{\frac{2 t+\epsilon}{1-(2 t+\epsilon)}} \leq|\mathfrak{N}| \leq c(\Omega)^{\frac{1-(2 t+\epsilon)}{1+2 t+\epsilon}} .
$$

This result clearly beats the convexity bound $L_{f i n}\left(1 / 2, \pi \times \sigma_{\Omega}\right) \ll_{\epsilon}\left(d_{E / \mathbf{Q}} c(\Omega) N\right)^{\frac{1}{2}+\epsilon}$ for all $\epsilon>0$. Similar results have been obtained in [MR], where $\Omega$ is a character of the ideal class group of $E$ and $N$ and $E$ vary. We remark that Michel and Harcos ([HM06] and [Mic04]) have proven subconvexity in the level aspect for $L_{\text {fin }}\left(1 / 2, \pi_{1} \times \pi_{2}\right)$ where $\pi_{1}$ and $\pi_{2}$ are cusp forms on GL(2)/Q with $\pi_{2}$ fixed. We also mention the work of Cogdell, Piatetski-Shapiro and Sarnak [Cog03] which proves subconvexity for the central value of a fixed Hilbert modular form twisted by a ray class character.

\subsection{About the proof}

Before continuing we give some background about the tools used in the proofs of the results contained in this paper. 


\subsubsection{Waldspurger's result}

An important result of Waldspurger [Wal85] relates $L\left(1 / 2, \pi_{E} \otimes \Omega\right)$ to period integrals of automorphic forms over the torus $E^{\times}$. More precisely let $X(\pi, E)$ be the set of isomorphism classes of quaternion algebras $D / F$ such that $E \hookrightarrow D$ and such that $\pi$ comes from an automorphic representation $\pi^{D}$ of $D^{\times}$via the Jacquet-Langlands correspondence. We note that $X(\pi, E)$ is finite, since $D$ must be isomorphic to the matrix algebra at all places where $\pi$ is unramified, and non-empty, since $M(2, F) \in X(\pi, E)$.

Take $D \in X(\pi, E)$ and let $\varphi$ be an element in the space of $\pi^{D}$. Thus, $\varphi$ is a function on $D^{\times} \backslash D^{\times}\left(\mathbf{A}_{F}\right)$ and we can define period integrals by

$$
P_{D}(\varphi)=\int_{E \times \mathbf{A}_{F}^{\times} \backslash \mathbf{A}_{E}^{\times}} \varphi(t) \Omega^{-1}(t) d t
$$

with $\mathbf{A}_{E}^{\times}$viewed as a subgroup of $D^{\times}\left(\mathbf{A}_{F}\right)$ via the inclusion $E \hookrightarrow D$. In [Wal85, Proposition 7], $\left|P_{D}(\varphi)\right|^{2}$ is expressed in terms of $L\left(1 / 2, \pi_{E} \otimes \Omega\right)$. For suitable choices of measure Waldspurger proves that,

$$
\frac{\left|P_{D}(\varphi)\right|^{2}}{(\varphi, \varphi)}=\frac{L\left(1 / 2, \pi_{E} \otimes \Omega\right)}{2 L(1, \pi, A d)} \prod_{v} \alpha_{v}\left(E_{v}, \varphi_{v}, \Omega_{v}\right)
$$

where $\alpha_{v}\left(E_{v}, \varphi_{v}, \Omega_{v}\right)$ are local period integrals which are equal to 1 for almost all $v$. Subsequent refinements of Waldspurger's result, [Gro87], [Zha01], [Xue06], [Pop06], [MW09], have sought to compute these local period integrals so as to provide an exact formula relating the $L$-value and the period integral. Under certain additional ramification conditions these results take the form,

$$
\frac{\left|P_{D}\left(\varphi_{\pi}\right)\right|^{2}}{\left(\varphi_{\pi}, \varphi_{\pi}\right)}=C(E, \pi, \Omega) L\left(1 / 2, \pi_{E} \otimes \Omega\right)
$$

where $D$ is a suitable element of $X(\pi, E)$ and $\varphi_{\pi} \in \pi^{D}$ is a test vector defined by Gross and Prasad [GP91]. The constant $C(E, \pi, \Omega)$ is of the form,

$$
C(E, \pi, \Omega)=\frac{1}{L(1, \pi, A d)} \frac{\sqrt{\Delta_{F}}}{2 \sqrt{c(\Omega) \Delta_{E}}} \prod_{v} C_{v}(E, \pi, \Omega)
$$

where the product is taken over the places $v$ of $F$ which are "bad" for $\pi$ or $\Omega$ and $C_{v}(E, \pi, \Omega)$ consists of certain local $L$-factors.

\subsubsection{Relative trace formula}

Having fixed the extension $E / F$ let $D / F$ be a quaternion algebra such that $E \hookrightarrow D$. We set $G$ equal to the group $P D^{\times}$over $F$ and let $T$ be the torus in $G$ such that $T(F)$ is equal to the image of $E^{\times}$in $G(F)$.

Let $f \in C_{c}^{\infty}\left(G\left(\mathbf{A}_{F}\right)\right)$. Then we have the map

$$
R(f): L^{2}\left(G(F) \backslash G\left(\mathbf{A}_{F}\right)\right) \rightarrow L^{2}\left(G(F) \backslash G\left(\mathbf{A}_{F}\right)\right)
$$


given by

$$
(R(f) \varphi)(x)=\int_{G\left(\mathbf{A}_{F}\right)} f(y) \varphi(x y) d y .
$$

$R(f)$ is an integral operator with kernel

$$
K_{f}(x, y)=\sum_{\gamma \in G(F)} f\left(x^{-1} \gamma y\right) .
$$

When $D$ is a division algebra, as a representation of $G\left(\mathbf{A}_{F}\right)$,

$$
L^{2}\left(G(F) \backslash G\left(\mathbf{A}_{F}\right)\right)=\bigoplus_{\pi \in \mathcal{A}(G)} \pi
$$

with the sum taken over the set of irreducible automorphic representations $\mathcal{A}(G)$ of $G\left(\mathbf{A}_{F}\right)$. Since $R(f)$ preserves each of the spaces $\pi$, the kernel has a spectral expansion

$$
K_{f}(x, y)=\sum_{\pi \in \mathcal{A}(G)} \sum_{\varphi \in \mathcal{B}(\pi)}(R(f) \varphi)(x) \overline{\varphi(y)}
$$

where $\mathcal{B}(\pi)$ denotes an orthonormal basis of the space of $\pi$.

Let $I(f)$ be the distribution defined by

$$
I(f)=\int_{T(F) \backslash T\left(\mathbf{A}_{F}\right)} \int_{T(F) \backslash T\left(\mathbf{A}_{F}\right)} K_{f}\left(t_{1}, t_{2}\right) \Omega\left(t_{1}^{-1} t_{2}\right) d t_{1} d t_{2} .
$$

The spectral expansion for $K_{f}(x, y)$ gives,

$I(f)=\sum_{\pi \in \mathcal{A}(G)} \sum_{\varphi \in \mathcal{B}(\pi)} \int_{T(F) \backslash T\left(\mathbf{A}_{F}\right)}(R(f) \varphi)\left(t_{1}\right) \Omega^{-1}\left(t_{1}\right) d t_{1} \bar{\int}_{T(F) \backslash T\left(\mathbf{A}_{F}\right)}^{\varphi\left(t_{2}\right) \Omega^{-1}\left(t_{2}\right) d t_{2}}$.

From the geometric expansion for the kernel, and after interchanging summation and integration,

$I(f)=\sum_{\gamma \in T(F) \backslash G(F) / T(F)} \operatorname{vol}\left(T_{\gamma}(F) \backslash T_{\gamma}\left(\mathbf{A}_{F}\right)\right) \int_{T_{\gamma}\left(\mathbf{A}_{F}\right) \backslash\left(T\left(\mathbf{A}_{F}\right) \times T\left(\mathbf{A}_{F}\right)\right)} f\left(t_{1}^{-1} \gamma t_{2}\right) \Omega\left(t_{1}^{-1} t_{2}\right) d t_{1} d t_{2}$,

where, for $\gamma \in G(F)$,

$$
T_{\gamma}=\left\{\left(t_{1}, t_{2}\right) \in T \times T: t_{1}^{-1} \gamma t_{2}=\gamma\right\} .
$$

We now return to the setting of the previous section. Having fixed $\mathfrak{N}$ we let $D$ be the quaternion algebra over $F$ which is ramified at the primes dividing $\mathfrak{N}$ and all the infinite places of $F$. Since the extension $E / F$ is inert at all the places of ramification for $D$ there exists an embedding $E \hookrightarrow D$. For each $\pi \in \mathcal{F}(\mathfrak{N}, 2 \boldsymbol{k})$ we let $\varphi_{\pi}$ be an element in the space of $\pi^{D}$ as in (1) above. We can choose a test function $f=f_{\mathfrak{N}, k}$ in $C_{c}^{\infty}\left(G\left(\mathbf{A}_{F}\right)\right)$, such that the operator $R(f)$ projects $L^{2}\left(G(F) \backslash G\left(\mathbf{A}_{F}\right)\right)$ onto the span of the set $\left\{\varphi_{\pi} \in \pi^{D}: \pi \in \mathcal{F}(\mathfrak{N}, 2 \boldsymbol{k})\right\}$. (This 
isn't quite true if all $k_{v}=1$, in this case the function $f_{\mathfrak{N}, k}$ may also pick out some 1-dimensional representations as well.) Hence for this function,

$$
I\left(f_{\mathfrak{N}, k}\right)=\sum_{\pi \in \mathcal{F}(\mathfrak{N}, 2 \boldsymbol{k})} \frac{\left|\int_{T(F) \backslash T\left(\mathbf{A}_{F}\right)} \varphi_{\pi}(t) \Omega^{-1}(t) d t\right|^{2}}{\left(\varphi_{\pi}, \varphi_{\pi}\right)} .
$$

Applying the identity (1) for each $\pi \in \mathcal{F}(\mathfrak{N}, 2 \boldsymbol{k})$ we get,

$$
I\left(f_{\mathfrak{N}, \boldsymbol{k}}\right)=C(E, \Omega, \boldsymbol{k}, \mathfrak{N}) \sum_{\pi \in \mathcal{F}(\mathfrak{N}, 2 k)} \frac{L\left(1 / 2, \pi_{E} \otimes \Omega\right)}{L(1, \pi, A d)},
$$

with $C(E, \Omega, \boldsymbol{k}, \mathfrak{N})$ an explicit constant. From the geometric expansion for $I\left(f_{\mathfrak{N}, \boldsymbol{k}}\right),(2)$, we obtain a closed expression for this average in terms of orbital integrals of the function $f_{\mathfrak{N}, k}$ over double cosets. Furthermore one can show that only finitely many $T(F)$ double cosets contribute to the sum. The results of this paper then stem from calculations of these geometric terms. When the level $\mathfrak{N}$ is large we show that only the identity double coset contributes which gives an exact formula for the average (Theorem 6.1), when the character $\Omega$ is everywhere unramified we can compute all the necessary orbital integrals (Theorem 6.5) and when the character $\Omega$ ramifies we are able to bound the terms on the geometric side of the trace formula which we can use towards the subconvexity problem for these $L$-values (Theorem 6.8).

\subsubsection{The case of modular forms of weight 2}

When $F=\mathbf{Q}$ the results of this paper are rephrased classically in Section 6.3. Here we illustrate our methods for modular forms of weight 2 and prime level in more classical language.

We fix an imaginary quadratic extension $E=\mathbf{Q}(\sqrt{-d})$ and take $N$ to be a prime which is inert and unramified in $E$. We take $\Omega$ to be a character of $\operatorname{Pic}(E)$, the ideal class group of $E$. Let $D$ be the quaternion algebra over $\mathbf{Q}$ which is ramified at $N$ and $\infty$. We fix an embedding $E \hookrightarrow D$ and take $R$ to be a maximal order in $D$ such that $R \cap E=\mathcal{O}_{E}$. Let $X$ denote the finite set of left equivalence classes of right $R$-ideals; see [Gro87, Section 1]. Given an ideal $\mathfrak{a}$ in $E$ we may form the right $R$-ideal $\mathfrak{a} R$ in $D$. In this way we get a well defined map $\iota: \operatorname{Pic}(E) \rightarrow X$. Let $F(X)$ denote the space of complex valued functions on $X$ which can be endowed with a natural inner product and an action of Hecke operators.

The Jacquet-Langlands correspondence, in this case, gives a Hecke-equivarient isomorphism JL : $F(X) \stackrel{\sim}{\longrightarrow} M_{2}(N)$ of $F(X)$ with the space of modular forms of weight 2 and level $N$; see [Gro87, Section 5]. We set $S(X)=\mathrm{JL}^{-1}\left(S_{2}(N)\right)$ where $S_{2}(N)$ denotes the space of cusp forms of level $N$ and weight 2 . The orthogonal complement of $S(X)$ in $F(X)$ is the space of constant functions.

We identify $\mathcal{F}(N, 2)$ with the set of normalized eigenforms in $S_{2}(N)$. For $f \in \mathcal{F}(N, 2)$ let $f^{\prime} \in F(X)$ be such that $\operatorname{JL}\left(f^{\prime}\right)=f$. Then the period relation 
for the central $L$-value can be expressed as,

$$
\frac{L\left(1, f \times g_{\Omega}\right)}{(f, f)}=C(N, E) \frac{\left|\sum_{y \in \operatorname{Pic}(E)} f^{\prime}(\iota(y)) \Omega(y)\right|^{2}}{\left(f^{\prime}, f^{\prime}\right)},
$$

where $g_{\Omega}$ is the $\theta$-series associated to $\Omega$ and $L\left(1, f \times g_{\Omega}\right)$ denotes the central value of the Rankin-Selberg $L$-function of $f$ with $g_{\Omega}$. Here $C(N, E)$ is an explicit constant depending only on $N$ and $E$. Thus,

$$
\sum_{f \in \mathcal{F}(N, 2)} \frac{L\left(1, f \times g_{\Omega}\right)}{(f, f)}=C(N, E) \sum_{f \in \mathcal{F}(N, 2)} \frac{\left|\sum_{y \in \operatorname{Pic}(E)} f^{\prime}(\iota(y)) \Omega(y)\right|^{2}}{\left(f^{\prime}, f^{\prime}\right)} .
$$

Since the orthogonal complement of $S(X)$ is spanned by the constant functions we see that if $\mathcal{B}$ is any orthonormal basis of $F(X)$ then

$$
\sum_{f \in \mathcal{F}(N, 2)} \frac{L\left(1, f \times g_{\Omega}\right)}{(f, f)}+C(N, E) \delta_{\Omega} \frac{\# \operatorname{Pic}(E)^{2}}{(\mathbf{1}, \mathbf{1})}=C(N, E) \sum_{h \in \mathcal{B}}\left|\sum_{y \in \operatorname{Pic}(E)} h(\iota(y)) \Omega(y)\right|^{2},
$$

where 1 denotes the function which is identically 1 on $X$, and

$$
\delta_{\Omega}= \begin{cases}1, & \text { if } \Omega \text { is trivial } \\ 0, & \text { otherwise }\end{cases}
$$

For each $x \in X$ let $\delta_{x} \in F(X)$ be the function defined by

$$
\delta_{x}(y)= \begin{cases}1, & \text { if } y=x \\ 0, & \text { otherwise }\end{cases}
$$

Now suppose we take for $\mathcal{B}$ the set $\left\{\delta_{x} /\left\|\delta_{x}\right\|: x \in X\right\}$. Then,

$$
\begin{aligned}
\sum_{h \in \mathcal{B}}\left|\sum_{y \in \operatorname{Pic}(E)} h(\iota(x)) \Omega(x)\right|^{2} & =\sum_{x \in X} \sum_{y_{1} \in \operatorname{Pic}(E)} \sum_{y_{2} \in \operatorname{Pic}(E)} \frac{\delta_{x}\left(\iota\left(y_{1}\right)\right) \delta_{x}\left(\iota\left(y_{2}\right)\right)}{\left\|\delta_{x}\right\|^{2}} \Omega\left(y_{1} y_{2}^{-1}\right) \\
& =\sum_{y_{1} \in \operatorname{Pic}(E)} \sum_{y_{2} \in \operatorname{Pic}(E)}\left(\frac{\delta_{x}\left(\iota\left(y_{1}\right)\right) \delta_{x}\left(\iota\left(y_{2}\right)\right)}{\left\|\delta_{x}\right\|^{2}}\right) \Omega\left(y_{1} y_{2}^{-1}\right) .
\end{aligned}
$$

For $y_{1}, y_{2} \in \operatorname{Pic}(E)$ clearly,

$$
\sum_{x \in X} \delta_{x}\left(\iota\left(y_{1}\right)\right) \delta_{x}\left(\iota\left(y_{2}\right)\right)= \begin{cases}1, & \text { if } \iota\left(y_{1}\right)=\iota\left(y_{2}\right) ; \\ 0, & \text { otherwise. }\end{cases}
$$

Let $\mathfrak{a}_{y}$ denote an ideal of $E$ which lies in the class of $y \in \operatorname{Pic}(E)$. Then $\iota\left(y_{1}\right)=$ $\iota\left(y_{2}\right)$ if and only if there exists $\gamma$, well defined in $E^{\times} \backslash D^{\times} / E^{\times}$, such that $\gamma \mathfrak{a}_{y_{1}} R=$ $\mathfrak{a}_{y_{2}} R$. For each $\gamma \in E^{\times} \backslash D^{\times} / E^{\times}$let,

$$
S(\gamma)=\left\{\left(y_{1}, y_{2}\right) \in \operatorname{Pic}(E) \times \operatorname{Pic}(E): \gamma \mathfrak{a}_{y_{1}} R=\mathfrak{a}_{y_{2}} R\right\} .
$$


Hence,

$$
\sum_{h \in \mathcal{B}}\left|\sum_{x \in \operatorname{Pic}(E)} h(\iota(x)) \Omega(x)\right|^{2}=\sum_{\gamma \in E^{\times} \backslash D^{\times} / E^{\times}} \sum_{\left(y_{1}, y_{2}\right) \in S(\gamma)} \frac{\Omega\left(y_{1} y_{2}^{-1}\right)}{\left\|\delta_{\iota\left(y_{1}\right)}\right\|^{2}},
$$

and so we deduce that

$$
\sum_{f \in \mathcal{F}(N, 2)} \frac{L\left(1, f \times g_{\Omega}\right)}{(f, f)}+C(N, E) \delta_{\mathbf{1}, \Omega} \frac{\# \operatorname{Pic}(E)^{2}}{(\mathbf{1}, \mathbf{1})}
$$

equals

$$
C(N, E)\left(\sum_{\gamma \in E^{\times} \backslash D^{\times} / E^{\times}} \sum_{\left(y_{1}, y_{2}\right) \in S(\gamma)} \frac{\Omega\left(y_{1} y_{2}^{-1}\right)}{\left\|\delta_{\iota\left(y_{1}\right)}\right\|^{2}}\right) .
$$

This is precisely the same identity, albeit without the adelic language, obtained via the relative trace formula. Furthermore it is clear that if $\iota: \operatorname{Pic}(E) \rightarrow X$ is injective then $S(\gamma)$ is empty unless $\gamma$ is the identity coset in $E^{\times} \backslash D^{\times} / E^{\times}$. The fact that $\iota$ is injective for $N$ sufficiently large then implies the stability result (Theorem 6.1) for the sum of the $L$-values which we obtain via the relative trace formula. For the application to subconvexity (Theorem 6.8) we need to provide bounds on the terms appearing in the geometric expansion. These we obtain from local calculations of the orbital integrals.

A similar situation has been studied in this setting by Michel and Venkatesh [MV07, Section 3]. In their work the form $f$ is fixed and $\Omega$ is allowed to vary over all characters of $\operatorname{Pic}(E)$. By averaging the period formula one obtains,

$$
\sum_{\Omega \in \widehat{\operatorname{Pic}(E)}} \frac{L\left(1, f \times g_{\Omega}\right)}{(f, f)}=C(N, E) \sum_{y \in \operatorname{Pic}(E)} \frac{\left|f^{\prime}(\iota(y))\right|^{2}}{\left(f^{\prime}, f^{\prime}\right)} .
$$

Analogously to the case considered here, when the discriminant of $E$ is large relative to the level of $f$ this formula can be made exact; see [MV07, Remark $3.1]$.

\subsection{About the paper}

\subsubsection{Summary of conditions}

Throughout this paper we enforce certain conditions on the level $\mathfrak{N}$ and weights $2 \boldsymbol{k}$ of our Hilbert modular forms as well as on the extension $E$ and the character $\Omega$. We now describe these conditions.

The ideal $\mathfrak{N}$ of $F$ is assumed to be squarefree and such that each prime divisor of $\mathfrak{N}$ is inert and unramified in $E$. This is, for the most part, a technical assumption so that we avoid the problem of having to deal with oldforms. This condition can most likely be removed with some extra work. We also assume that the number of prime divisors of $\mathfrak{N}$ has the same parity as $[F: \mathbf{Q}]$, this is 
a necessary condition as it ensures that the sign of the functional equation of $L\left(s, \pi_{E} \otimes \Omega\right)$ is +1 .

We place certain restrictions on the character $\Omega$ as well. We assume that $\Omega$ is unramified above $\mathfrak{N}$. This assumption is needed in the work of Gross and Prasad [GP91] which provides the test vector $\varphi_{\pi}$ which appears in (1). We also assume, at the archimedean places, that the weights of $\Omega$ are strictly smaller than the weights $k$ of the Hilbert modular forms. This ensures that the quaternion algebra $D$ which appears in (1) is ramified at the archimedean places of $F$. Without this assumption the function $f_{\mathfrak{N}, k}$ would not be compactly supported and one would not obtain a finite closed formula for the average $L$ values. In this case it is likely that one could work out an asymptotic version of the formulas in this paper. This would require some additional archimedean calculations similar to [RR05, Section 2].

Finally it would also be interesting to consider the case of Maass forms. It would seem that again with some additional archimedean calculations this could be carried out.

\subsubsection{Outline of the paper}

This paper is set up as follows. We begin by introducing our notation and defining our measures. In the following section we pick our test functions and compute their spectral expansion in the relative trace formula. In the fourth section we explicitly compute the geometric side of the relative trace formula for these test functions. While, a priori, there are an infinite number of orbital integrals that appear in the geometric expansion, we show that for our test functions only a finite number of terms are nonzero. This is what allows us to get an exact, rather than asymptotic, formula.

In the fifth section we compute a distribution on the Hecke algebra which appears in the geometric expansion of the relative trace formula. In the last section we combine the spectral and geometric calculations to get our main formulas. We also establish our application to subconvexity and rewrite our formulae over $\mathbf{Q}$ in classical language.

The original motivation for our work came from trying to understand [RR05], which applies the relative trace formula of [Jac86] to average values of base change $L$-functions. We found that by integrating over nonsplit rather than split tori, and applying [MW09], we obtain more general and exact results. Furthermore, by working on quaternion algebras we avoid having to deal with contributions from oldforms.

\subsection{Acknowledgements}

We wish to thank Peter Sarnak for his valuable suggestions and encouragement. We thank Dinakar Ramakrishnan for his useful comments, as well as furnishing us with early versions of his work with Philippe Michel [MR]. We also thank Dipendra Prasad for helpful conversations and the Institute for Advanced Study 
for a stimulating mathematical environment. Finally, we thank the referees for their helpful comments.

The first author was supported by NSF grant DMS-0111298 and the Natural Sciences and Engineering Research Council of Canada. The second author was supported by NSF grants DMS-0111298 and DMS-0758197. Any opinions, findings and conclusions or recommendations expressed in this material are those of the authors and do not necessarily reflect the views of the National Science Foundation.

\section{Notation}

Let $F$ be a totally real number field of discriminant $\Delta_{F}$ and class number $h_{F}$. For a finite place $v$ of $F, \varpi_{v}$ denotes a uniformizing parameter in $F_{v}$ and $q_{v}$ denotes the cardinality of the residue class field at $v$. The ring of integers in $F_{v}$ is denoted by $\mathcal{O}_{F_{v}}$, the units by $U_{F_{v}}$ and for $n>0$ we set $U_{F_{v}}^{n}=1+\varpi_{v}^{n} \mathcal{O}_{F_{v}}$. For an ideal $\mathfrak{a}$ of $\mathcal{O}_{F}$, we denote by $|\mathfrak{a}|$ the absolute norm of $\mathfrak{a}$. We denote by $\Sigma_{\infty}$ the set of infinite places of $F$. We take $\mathfrak{N}$ to be a squarefree ideal in $\mathcal{O}_{F}$ such that the number of primes dividing $\mathfrak{N}$ has the same parity as $[F: \mathbf{Q}]$. For each place $v \in \Sigma_{\infty}$ we fix an integer $k_{v} \geq 1$. We denote by $\mathcal{F}(\mathfrak{N}, 2 \boldsymbol{k})$ the set of cuspidal automorphic representations of $\operatorname{PGL}\left(2, \mathbf{A}_{F}\right)$ of level $\mathfrak{N}$ and weight $2 \boldsymbol{k}=\left(2 k_{v}\right)$.

We now take a $\mathrm{CM}$ extension $E / F$ such that each prime of $F$ dividing $\mathfrak{N}$ is inert and unramified in $E$. We let $\mathfrak{D}_{E / F}$ be the different of $E / F, \mathfrak{d}_{E / F}$ be the discriminant of $E / F$ and $d_{E / F}=\left|\mathfrak{d}_{E / F}\right|$. We denote by $\eta$ the quadratic character of $F^{\times} \backslash \mathbf{A}_{F}^{\times}$associated to $E / F$ by class field theory and by $N$ the norm map from $E$ to $F$. For a place $v$ of $F$ we denote by $E_{v}=E \otimes_{F} F_{v}$, by $\mathcal{O}_{E_{v}}$ the integral closure of $\mathcal{O}_{F_{v}}$ in $E_{v}$ and by $U_{E_{v}}=\mathcal{O}_{E_{v}}^{\times}$. We denote the action of the non-trivial element in $\operatorname{Gal}(E / F)$ on $\alpha \in E_{v}$ by $\bar{\alpha}$.

We take a unitary character $\Omega: E^{\times} \backslash \mathbf{A}_{E}^{\times} \rightarrow \mathbf{C}^{\times}$such that $\left.\Omega\right|_{\mathbf{A}_{F}^{\times}}$is trivial. We assume that $\Omega$ is unramified above $\mathfrak{N}$ and that at each place $v \in \Sigma_{\infty}, \Omega_{v}$ has the form

$$
z \mapsto\left(\frac{z}{\bar{z}}\right)^{m_{v}}
$$

with $\left|m_{v}\right|$ strictly less than $k_{v}$. We set $\boldsymbol{m}=\left(m_{v}\right)$. At each finite place $v$ of $F$ we denote by $n\left(\Omega_{v}\right)$ the smallest non-negative integer such that $\Omega$ is trivial on $\left(\mathcal{O}_{F}+\varpi_{v}^{n\left(\Omega_{v}\right)} \mathcal{O}_{E}\right)^{\times}$. We denote by $\mathfrak{c}(\Omega)$ the norm of the conductor of $\Omega$ in $F$ and by $c(\Omega)$ the absolute norm of $\mathfrak{c}(\Omega)$. We note that $c(\Omega)=\prod_{v<\infty} q_{v}^{2 n\left(\Omega_{v}\right)}$. We use $S(\Omega)$ to denote the set of places of $F$ above which $\Omega$ is ramified.

We define

$$
\left(\begin{array}{c}
2 \boldsymbol{k}-2 \\
\boldsymbol{k}+\boldsymbol{m}-1
\end{array}\right)=\prod_{v \in \Sigma_{\infty}}\left(\begin{array}{c}
2 k_{v}-2 \\
k_{v}+m_{v}-1
\end{array}\right)
$$

Having fixed $\mathfrak{N}$, we let $D$ be the quaternion algebra defined over $F$ which is ramified precisely at the infinite places of $F$ and the places dividing $\mathfrak{N}$. We fix an embedding $E \hookrightarrow D$. We let $Z$ denote the center of $D$ and denote by $G$ 
the group $Z \backslash D^{\times}$viewed as an algebraic group defined over $F$. We denote by $N_{D}: D^{\times} \rightarrow F^{\times}$the reduced norm. At each finite place $v$ of $F$ we fix a maximal order $R_{v}$ in $D_{v}$ such that $R_{v} \cap E_{v}=\mathcal{O}_{F_{v}}+\varpi_{v}^{n\left(\Omega_{v}\right)} \mathcal{O}_{E_{v}}$. We note that $R_{v}$ is well defined up to $E_{v}^{\times}$conjugacy.

All $L$-functions are completed.

\subsection{Normalization of measures}

We fix an additive character $\psi$ of $F \backslash \mathbf{A}_{F}$ which, for the sake of convenience, we take to be $\psi=\psi_{0} \circ \operatorname{tr}_{F / \mathbf{Q}}$ where $\psi_{0}$ denotes the standard character on $\mathbf{Q} \backslash \mathbf{A}_{\mathbf{Q}}$. For a place $v$ of $F$ we take the additive Haar measure $d x$ on $F_{v}$ which is self-dual with respect to $\psi_{v}$. On $F_{v}^{\times}$we take the measure

$$
d^{\times} x=L\left(1,1_{F_{v}}\right) \frac{d x}{|x|_{v}} .
$$

We define measures on $E_{v}$ and $E_{v}^{\times}$similarly with respect to the additive character $\psi \circ \operatorname{tr}_{E / F}$. We note that with these choices of measures we have

$$
\prod_{v<\infty} \operatorname{vol}\left(U_{F_{v}}, d^{\times} x_{v}\right)=\left|\Delta_{F}\right|^{-\frac{1}{2}}
$$

and similarly for $E$. We also have

$$
\operatorname{vol}\left(F_{v}^{\times} \backslash E_{v}^{\times}\right)=\operatorname{vol}\left(\mathbf{R}^{\times} \backslash \mathbf{C}^{\times}\right)=2
$$

for $v \in \Sigma_{\infty}$.

For the group $D$ we recall that there exists an $\epsilon \in F^{\times}$, well defined in $F^{\times} / N E^{\times}$, such that $D$ is isomorphic to

$$
\left\{\left(\begin{array}{cc}
\alpha & \epsilon \beta \\
\bar{\beta} & \bar{\alpha}
\end{array}\right): \alpha, \beta \in E\right\}
$$

For a place $v$ of $F$ we take the Tamagawa measure $d g_{v}$ on $D_{v}^{\times}$which is given by

$$
d g_{v}=L\left(1,1_{F_{v}}\right)|\epsilon|_{v} \frac{d \alpha_{v} d \beta_{v}}{\left|\alpha_{v} \bar{\alpha}_{v}-\epsilon \beta_{v} \bar{\beta}_{v}\right|_{v}^{2}}
$$

We note that this measure only depends on the choice of $\epsilon$ modulo $N E^{\times}$. Furthermore with this definition

$$
\operatorname{vol}\left(R_{v}^{\times}\right)=L\left(2,1_{F_{v}}\right)^{-1} \operatorname{vol}\left(U_{F_{v}}, d^{\times} x_{v}\right)^{4}
$$

for non-archimedean $v \nmid \mathfrak{N}$ since the isomorphism of $D_{v}^{\times}$with $\mathrm{GL}\left(2, F_{v}\right)$ preserves Tamagawa measures. We have

$$
\operatorname{vol}\left(R_{v}^{\times}\right)=\frac{L\left(2,1_{F_{v}}\right)^{-1}}{q_{v}-1} \operatorname{vol}\left(U_{F_{v}}, d^{\times} x_{v}\right)^{4}
$$


for $v \mid \mathfrak{N}$ by a straightforward calculation. We also note that

$$
\operatorname{vol}\left(G\left(F_{v}\right)\right)=4 \pi^{2}
$$

for $v \in \Sigma_{\infty}$.

Globally we take the product of these local measures and give discrete subgroups the counting measures. In this way we get

$$
\operatorname{vol}\left(\mathbf{A}_{F}^{\times} E^{\times} \backslash \mathbf{A}_{E}^{\times}\right)=2 L(1, \eta)
$$

and

$$
\operatorname{vol}\left(G(F) \backslash G\left(\mathbf{A}_{F}\right)\right)=2 .
$$

\section{Spectral side of the trace formula}

Having fixed the quaternion algebra $D$ we have taken $G$ to be the algebraic group defined over $F$ with $G(F)=D^{\times} / F^{\times}$. Since $D$ is anisotropic the quotient $G(F) \backslash G\left(\mathbf{A}_{F}\right)$ is compact and hence, as a representation of $G\left(\mathbf{A}_{F}\right)$,

$$
L^{2}\left(G(F) \backslash G\left(\mathbf{A}_{F}\right)\right)=\widehat{\bigoplus}_{\pi^{\prime} \in \mathcal{A}(G)} V_{\pi^{\prime}} .
$$

Here the sum is taken over the irreducible automorphic representations $\mathcal{A}(G)$ of $G\left(\mathbf{A}_{F}\right)$ and for each $\pi^{\prime} \in \mathcal{A}(G), V_{\pi^{\prime}}$ denotes the space of $\pi^{\prime}$. The JacquetLanglands correspondence [JL70] yields an injection JL : $\mathcal{A}(G) \hookrightarrow \mathcal{A}(\mathrm{PGL}(2))$, where $\mathcal{A}(\mathrm{PGL}(2))$ denotes the set of automorphic representations of $\mathrm{PGL}\left(2, \mathbf{A}_{F}\right)$. The set $\mathcal{A}(G)$ can be decomposed as,

$$
\mathcal{A}(G)=\mathcal{A}_{\text {cusp }}(G) \amalg \mathcal{A}_{\text {res }}(G),
$$

where

$$
\mathcal{A}_{\text {cusp }}(G)=\left\{\pi^{\prime} \in \mathcal{A}(G): \operatorname{JL}\left(\pi^{\prime}\right) \text { is cuspidal }\right\}
$$

and

$$
\mathcal{A}_{\text {res }}(G)=\left\{\delta \circ N_{D}: \delta: F^{\times} \backslash \mathbf{A}_{F}^{\times} \rightarrow\{ \pm 1\}\right\} .
$$

The compatibility between the local and global Jacquet-Langlands correspondence yields the following.

Fact 3.1. The image of $\mathcal{A}_{\text {cusp }}(G)$ under JL is equal to the set of cuspidal automorphic representations $\pi=\otimes_{v} \pi_{v}$ of $\mathrm{PGL}\left(2, \mathbf{A}_{F}\right)$ such that $\pi_{v}$ is a discrete series representation of $\operatorname{PGL}\left(2, F_{v}\right)$ at all places $v$ where $D$ ramifies. In particular $\mathcal{F}(\mathfrak{N}, 2 \boldsymbol{k})$ is contained in the image of JL and for $\pi^{\prime}=\otimes_{v} \pi_{v}^{\prime} \in \mathcal{A}_{\text {cusp }}(G)$ we have $\mathrm{JL}\left(\pi^{\prime}\right) \in \mathcal{F}(\mathfrak{N}, 2 \boldsymbol{k})$ if and only if,

1. for $v \mid \mathfrak{N}, \pi_{v}^{\prime} \cong \delta_{v} \circ N_{D_{v}}$ where $\delta_{v}: F_{v}^{\times} \rightarrow \mathbf{C}^{\times}$is an unramified character (necessarily of order at most 2$)$, 
2. for $v \in \Sigma_{\infty}, \pi_{v}^{\prime} \cong \operatorname{Sym}^{2 k_{v}-2} V \otimes \operatorname{det}^{1-k_{v}}$, where $V$ denotes the irreducible 2-dimensional representation of $D^{\times}\left(F_{v}\right)$ coming from the isomorphism $D^{\times}\left(\bar{F}_{v}\right) \stackrel{\sim}{\longrightarrow} \mathrm{GL}(2, \mathbf{C})$, and

3. for all other $v, \pi_{v}^{\prime}$ is unramified.

We set $\mathcal{F}^{\prime}(\mathfrak{N}, 2 \boldsymbol{k})=\left\{\pi^{\prime} \in \mathcal{A}_{\text {cusp }}(G): \operatorname{JL}\left(\pi^{\prime}\right) \in \mathcal{F}(\mathfrak{N}, 2 \boldsymbol{k})\right\}$.

Let $f \in C_{c}^{\infty}\left(G\left(\mathbf{A}_{F}\right)\right)$, integrating $f$ against the action of $G\left(\mathbf{A}_{F}\right)$ gives a linear map

$$
R(f): L^{2}\left(G(F) \backslash G\left(\mathbf{A}_{F}\right)\right) \rightarrow L^{2}\left(G(F) \backslash G\left(\mathbf{A}_{F}\right)\right)
$$

defined by

$$
(R(f) \varphi)(x)=\int_{G\left(\mathbf{A}_{F}\right)} f(g) \varphi(x g) d g .
$$

From the spectral decomposition of $L^{2}\left(G(F) \backslash G\left(\mathbf{A}_{F}\right)\right)$ one sees that $R(f)$ is an integral operator with kernel,

$$
K_{f}(x, y)=\sum_{\pi^{\prime} \in \mathcal{A}(G)} \sum_{\varphi \in \mathcal{B}\left(\pi^{\prime}\right)}(R(f) \varphi)(x) \overline{\varphi(y)},
$$

where for each $\pi^{\prime} \in \mathcal{A}(G), \mathcal{B}\left(\pi^{\prime}\right)$ denotes an orthonormal basis of $V_{\pi^{\prime}}$.

Having fixed the embedding $E \hookrightarrow D$ we get an injection $\mathbf{A}_{E}^{\times} \hookrightarrow D^{\times}\left(\mathbf{A}_{F}\right)$. We define a distribution

$$
I(f)=\int_{\mathbf{A}_{F}^{\times} E^{\times} \backslash \mathbf{A}_{E}^{\times}} \int_{\mathbf{A}_{F}^{\times} E^{\times} \backslash \mathbf{A}_{E}^{\times}} K_{f}\left(t_{1}, t_{2}\right) \Omega\left(t_{1}^{-1} t_{2}\right) d t_{1} d t_{2} .
$$

The spectral expansion for the kernel $K_{f}(x, y)$ gives,

$$
I(f)=\sum_{\pi^{\prime} \in \mathcal{A}(G)} \sum_{\varphi \in \mathcal{B}\left(\pi^{\prime}\right)} \int_{\mathbf{A}_{F}^{\times} E^{\times} \backslash \mathbf{A}_{E}^{\times}}(R(f) \varphi)\left(t_{1}\right) \Omega^{-1}\left(t_{1}\right) d t_{1} \int_{\mathbf{A}_{F}^{\times} E^{\times} \backslash \mathbf{A}_{E}^{\times}} \varphi\left(t_{2}\right) \Omega^{-1}\left(t_{2}\right) d t_{2} .
$$

For each $\pi^{\prime} \in \mathcal{A}(G)$ we define

$$
I_{\pi^{\prime}}(f)=\sum_{\varphi \in \mathcal{B}\left(\pi^{\prime}\right)} \int_{\mathbf{A}_{F}^{\times} E^{\times} \backslash \mathbf{A}_{E}^{\times}}(R(f) \varphi)\left(t_{1}\right) \Omega^{-1}\left(t_{1}\right) d t_{1} \int_{\mathbf{A}_{F}^{\times} E^{\times} \backslash \mathbf{A}_{E}^{\times}} \varphi\left(t_{2}\right) \Omega^{-1}\left(t_{2}\right) d t_{2} .
$$

We set

$$
I_{\text {cusp }}(f)=\sum_{\pi^{\prime} \in \mathcal{A}_{\text {cusp }}(G)} I_{\pi^{\prime}}(f)
$$

and

$$
I_{\text {res }}(f)=\sum_{\pi^{\prime} \in \mathcal{A}_{\text {res }}(G)} I_{\pi^{\prime}}(f)
$$

so that $I(f)=I_{\text {cusp }}(f)+I_{\text {res }}(f)$. In the same we also write

$$
K_{f}(x, y)=K_{f, c u s p}(x, y)+K_{f, r e s}(x, y) .
$$

Our goal in this section is to choose a suitable test function $f \in C_{c}^{\infty}\left(G\left(\mathbf{A}_{F}\right)\right)$ such that $R(f)$ kills all $\pi^{\prime} \in \mathcal{A}_{\text {cusp }}(G)$ such that $\operatorname{JL}\left(\pi^{\prime}\right) \notin \mathcal{F}(\mathfrak{N}, 2 \boldsymbol{k})$. We will then compute $I(f)$ and use a precise version of Waldspurger's formula [MW09, Theorem 4.1] to relate $I_{c u s p}(f)$ to the $L$-values being considered in this paper. 


\subsection{The test function}

We fix a finite prime $\mathfrak{p} \nmid \mathfrak{N}$ of $F$. We will consider test functions

$$
f=\prod_{v} f_{v} \in C_{c}^{\infty}\left(G\left(\mathbf{A}_{F}\right)\right)
$$

defined as follows.

At a finite place $v \neq \mathfrak{p}$ we take $f_{v}=\mathbf{1}_{v}$, the characteristic function of $Z_{v} R_{v}^{\times}$. At $v=\mathfrak{p}$ we allow $f_{\mathfrak{p}}$ to be any element in the Hecke algebra $\mathcal{H}\left(G\left(F_{\mathfrak{p}}\right), Z_{\mathfrak{p}} R_{\mathfrak{p}}^{\times}\right)$.

Let $v \in \Sigma_{\infty}$. We fix an isomorphism $D^{\times}\left(\overline{F_{v}}\right) \cong \operatorname{GL}(2, \mathbf{C})$ which gives an irreducible 2-dimensional representation $V$ of $D^{\times}\left(F_{v}\right)$. We set $\pi_{2 k_{v}}^{\prime}=$ $\operatorname{Sym}^{2 k_{v}-2} V \otimes \operatorname{det}^{-k_{v}+1}$, which descends to a well defined representation of $G\left(F_{v}\right)$ corresponding, via the local Jacquet-Langlands correspondence, to the weight $2 k_{v}$ discrete series on $\operatorname{PGL}(2, \mathbf{R})$. We note that $\operatorname{dim} \pi_{2 k_{v}}^{\prime}=2 k_{v}-1$. Let $\langle$, be a $G\left(F_{v}\right)$ invariant inner product of $\pi_{2 k_{v}}^{\prime}$. As is well known, since $\left|m_{v}\right|<k_{v}$ the subspace of $\pi_{2 k_{v}}^{\prime}$,

$$
\pi_{2 k_{v}}^{\prime}\left(\Omega_{v}\right)=\left\{w \in \pi_{2 k_{v}}^{\prime}: \pi_{2 k_{v}}^{\prime}(t) w=\Omega_{v}(t) w \text { for all } t \in E_{v}^{\times}\right\}
$$

is 1-dimensional. We fix a unit vector $w_{v} \in \pi_{2 k_{v}}^{\prime}\left(\Omega_{v}\right)$ and define $f_{v} \in C_{c}^{\infty}\left(G\left(F_{v}\right)\right)$ by

$$
f_{v}(g)=\overline{\left\langle\pi_{2 k_{v}}^{\prime}(g) w_{v}, w_{v}\right\rangle}
$$

Given $f_{\mathfrak{p}} \in \mathcal{H}\left(G\left(F_{\mathfrak{p}}\right), Z_{\mathfrak{p}} R_{\mathfrak{p}}^{\times}\right)$we denote by $\hat{f}_{\mathfrak{p}}$ the function on unramified representations $\pi_{\mathfrak{p}}$ defined by

$$
\hat{f}_{\mathfrak{p}}\left(\pi_{\mathfrak{p}}\right)=\frac{1}{\operatorname{vol}\left(Z_{\mathfrak{p}} \backslash Z_{\mathfrak{p}} R_{\mathfrak{p}}^{\times}\right)} \operatorname{Tr} \pi_{\mathfrak{p}}\left(f_{\mathfrak{p}}\right) .
$$

We also view $\hat{f}_{\mathfrak{p}}$ as a function on $[-2,2]$ in the usual way.

\subsection{Local preliminaries}

Before continuing onto the calculation of $I(f)$ for $f$ as in Section 3.1 we record some local preliminaries. For an irreducible admissible representation $\sigma$ of $G\left(F_{v}\right)$ acting on the space $V_{\sigma}$ and $f_{v} \in C_{c}^{\infty}\left(G\left(F_{v}\right)\right)$ we let,

$$
\sigma\left(f_{v}\right): V_{\sigma} \rightarrow V_{\sigma}: w \mapsto \int_{G\left(F_{v}\right)} f_{v}\left(g_{v}\right) \sigma\left(g_{v}\right) w d g_{v}
$$

We record the following basic results for use later.

Lemma 3.2. Let $v$ be a finite place of $F$ not dividing $\mathfrak{N}$ and let $f_{v} \in C_{c}^{\infty}\left(G\left(F_{v}\right)\right)$ be as in Section 3.1. Let $\sigma$ be an irreducible unitarizable representation of $G\left(F_{v}\right)$. Then $\sigma\left(f_{v}\right)$ kills the orthogonal complement of $\sigma^{R_{v}^{\times}}$in $V_{\sigma}$ and for $w \in \sigma^{R_{v}^{\times}}$,

$$
\sigma\left(f_{v}\right) w=\operatorname{vol}\left(Z_{v} \backslash Z_{v} R_{v}^{\times}\right) \hat{f}_{v}(\sigma) w .
$$

Furthermore $\sigma^{R_{v}^{\times}}$has dimension at most one. 
Proof. For $v \nmid \mathfrak{N}, G\left(F_{v}\right) \cong \operatorname{PGL}\left(2, F_{v}\right)$ and this result is well known.

Lemma 3.3. Let $v$ be a finite place of $F$ dividing $\mathfrak{N}$ and let $f_{v} \in C_{c}^{\infty}\left(G\left(F_{v}\right)\right)$ be as in Section 3.1. Let $\sigma$ be an irreducible unitarizable representation of $G\left(F_{v}\right)$. Then $\sigma\left(f_{v}\right)$ kills the orthogonal complement of $\sigma^{R_{v}^{\times}}$in $V_{\sigma}$ and for $w \in \sigma^{R_{v}^{\times}}$,

$$
\sigma\left(f_{v}\right) w=\operatorname{vol}\left(Z_{v} \backslash Z_{v} R_{v}^{\times}\right) w .
$$

Furthermore if $\sigma^{R_{v}^{\times}} \neq 0$ then $\sigma=\delta \circ N_{D_{v}}$ for an unramified character $\delta$ of $F_{v}^{\times}$.

Proof. The first part of the lemma is clear. It remains to prove the last statement. In this case we use the exact sequence,

$$
1 \longrightarrow R_{v}^{\times} \longrightarrow D_{v}^{\times} \stackrel{N_{D_{v}}}{\longrightarrow} F_{v}^{\times} / U_{F_{v}} \longrightarrow 1 .
$$

From which it follows that if $\sigma^{R_{v}^{\times}} \neq 0$ then $\sigma=\delta \circ N_{D_{v}}$ for a character $\delta$ of $F_{v}^{\times} / U_{F_{v}}$.

Lemma 3.4. Let $v$ be an infinite place of $F$ and let $f_{v} \in C_{c}^{\infty}\left(G\left(F_{v}\right)\right)$ be as in Section 3.1. Let $\sigma$ be an irreducible unitarizable representation of $G\left(F_{v}\right)$. Then $\sigma\left(f_{v}\right)$ kills the space of $\sigma$ unless $\sigma \cong \pi_{2 k_{v}}^{\prime}$. Furthermore for $\sigma=\pi_{2 k_{v}}^{\prime}$ the map $\pi_{2 k_{v}}^{\prime}\left(f_{v}\right)$ kills the orthogonal complement of $\pi_{2 k_{v}}^{\prime}\left(\Omega_{v}\right)$ and for $w \in \pi_{2 k_{v}}^{\prime}\left(\Omega_{v}\right)$,

$$
\pi_{2 k_{v}}^{\prime}\left(f_{v}\right) w=\frac{\operatorname{vol}\left(G\left(F_{v}\right)\right)}{2 k_{v}-1} w .
$$

Proof. Since $f_{v}$ is a matrix coefficient of $\pi_{2 k_{v}}^{\prime}$ it follows that $\sigma\left(f_{v}\right)$ kills the space of $\sigma$ unless $\sigma \cong \pi_{2 k_{v}}^{\prime}$. On the other hand if $0 \neq w \in \pi_{2 k_{v}}^{\prime}\left(\Omega_{v}\right)$ then

$$
f_{v}(g)=\frac{\overline{\left\langle\pi_{2 k_{v}}^{\prime}(g) w, w\right\rangle}}{\langle w, w\rangle} .
$$

Since $\pi_{2 k_{v}}^{\prime}\left(\Omega_{v}\right)$ is spanned by $w$ it follows that $\pi_{2 k_{v}}^{\prime}\left(f_{v}\right)$ kills the orthogonal complement of $\pi_{2 k_{v}}^{\prime}\left(\Omega_{v}\right)$ in $\pi_{2 k_{v}}^{\prime}$. Finally,

$$
\begin{aligned}
\left\langle\pi_{2 k_{v}}^{\prime}\left(f_{v}\right) w, w\right\rangle & =\int_{G\left(F_{v}\right)} f_{v}(g)\left\langle\pi_{2 k_{v}}^{\prime}(g) w, w\right\rangle d g \\
& =\int_{G\left(F_{v}\right)} \frac{\left|\left\langle\pi_{2 k_{v}}^{\prime}(g) w, w\right\rangle\right|^{2}}{\langle w, w\rangle} d g \\
& =\frac{\operatorname{vol}\left(G\left(F_{v}\right)\right)}{\operatorname{dim} \pi_{2 k_{v}}^{\prime}}\langle w, w\rangle .
\end{aligned}
$$

The last part of the Lemma now follows.

As a temporary expedient we set,

$$
\begin{aligned}
\lambda(\mathfrak{N}, \boldsymbol{k}) & =\prod_{v<\infty} \operatorname{vol}\left(Z_{v} \backslash R_{v}^{\times} Z_{v}\right) \prod_{v \in \Sigma_{\infty}} \frac{\operatorname{vol}\left(G\left(F_{v}\right)\right)}{2 k_{v}-1} \\
& =\frac{1}{\left|\Delta_{F}\right|^{\frac{3}{2}} L\left(2,1_{F}\right)} \prod_{v \mid \mathfrak{N}} \frac{1}{q_{v}-1} \prod_{v \in \Sigma_{\infty}} \frac{4 \pi}{2 k_{v}-1} .
\end{aligned}
$$




\subsection{Global calculations}

Having chosen $f \in C_{c}^{\infty}\left(G\left(\mathbf{A}_{F}\right)\right)$ in Section 3.1 we now set about computing

$$
I(f)=I_{\text {cusp }}(f)+I_{\text {res }}(f) .
$$

We begin with the calculation of $I_{\text {res }}(f)$ which, as we shall see, is often zero. We define $X^{u n}(F)$ to be the set of unramified characters $\chi: F^{\times} \backslash \mathbf{A}_{F}^{\times} \rightarrow\{ \pm 1\}$.

Lemma 3.5. For $f \in C_{c}^{\infty}\left(G\left(\mathbf{A}_{F}\right)\right)$ as in Section 3.1, $K_{f, r e s}(x, y) \equiv 0$ unless $2 k_{v}=2$ for all $v \in \Sigma_{\infty}$, in which case

$$
K_{f, r e s}(x, y)=\frac{\lambda(\mathfrak{N}, \boldsymbol{k})}{\operatorname{vol}\left(G(F) \backslash G\left(\mathbf{A}_{F}\right)\right)} \sum_{\delta \in X^{u n}(F)} \delta\left(N_{D}\left(x y^{-1}\right)\right) \hat{f}_{\mathfrak{p}}\left(\delta_{\mathfrak{p}} \circ N_{D}\right) .
$$

Proof. We recall that

$$
\mathcal{A}_{r e s}(G)=\left\{\delta \circ N_{D}: \delta: F^{\times} \backslash \mathbf{A}_{F}^{\times} \rightarrow\{ \pm 1\}\right\} .
$$

We fix a character $\delta: F^{\times} \backslash \mathbf{A}_{F}^{\times} \rightarrow\{ \pm 1\}$. By definition we have

$$
\begin{aligned}
\left(R(f)\left(\delta \circ N_{D}\right)\right)(x) & =\int_{G\left(\mathbf{A}_{F}\right)} f(g) \delta\left(N_{D}(x g)\right) d g \\
& =\prod_{v} \int_{G\left(F_{v}\right)} f_{v}\left(g_{v}\right) \delta_{v}\left(N_{D_{v}}\left(x g_{v}\right)\right) d g_{v}
\end{aligned}
$$

We now set about computing the local integrals. Suppose first that $v$ is a nonarchimedean place. If $v \neq \mathfrak{p}$ then $f_{v}$ is the characteristic function of $Z_{v} R_{v}^{\times}$. Hence,

$$
\int_{G\left(F_{v}\right)} f_{v}\left(g_{v}\right) \delta_{v}\left(N_{D_{v}}\left(x g_{v}\right)\right) d g_{v}=\delta_{v}\left(N_{D_{v}}(x)\right) \int_{Z_{v} \backslash Z_{v} R_{v}^{\times}} \delta_{v}\left(N_{D_{v}}\left(g_{v}\right)\right) d g_{v} .
$$

Since the norm map $N_{D_{v}}: R_{v}^{\times} \rightarrow U_{F_{v}}$ is surjective,

$$
\int_{Z_{v} \backslash Z_{v} R_{v}^{\times}} \delta_{v}\left(N_{D_{v}}\left(x g_{v}\right)\right) d g_{v}= \begin{cases}0, & \text { if } \delta_{v} \text { is ramified; } \\ \delta_{v}\left(N_{D_{v}}(x)\right) \operatorname{vol}\left(Z_{v} \backslash Z_{v} R_{v}^{\times}\right), & \text {if } \delta_{v} \text { is unramified. }\end{cases}
$$

If $v=\mathfrak{p}$, then

$$
\begin{aligned}
\int_{G\left(F_{v}\right)} f_{\mathfrak{p}}\left(g_{\mathfrak{p}}\right) \delta_{\mathfrak{p}}\left(N_{D_{\mathfrak{p}}}\left(x g_{\mathfrak{p}}\right)\right) d g_{\mathfrak{p}} & =\delta_{\mathfrak{p}}\left(N_{D_{\mathfrak{p}}}(x)\right) \int_{G\left(F_{\mathfrak{p}}\right)} f_{\mathfrak{p}}\left(g_{\mathfrak{p}}\right) \delta_{\mathfrak{p}}\left(N_{D_{\mathfrak{p}}}\left(g_{\mathfrak{p}}\right)\right) d g_{\mathfrak{p}} \\
& =\delta_{\mathfrak{p}}\left(N_{D_{\mathfrak{p}}}(x)\right) \operatorname{Tr}\left(\delta_{\mathfrak{p}} \circ N_{D_{\mathfrak{p}}}\right)\left(f_{\mathfrak{p}}\right) .
\end{aligned}
$$

Clearly, since $f_{\mathfrak{p}}$ is bi- $R_{\mathfrak{p}}^{\times}$-invariant we have $\operatorname{Tr}\left(\delta_{\mathfrak{p}} \circ N_{D_{\mathfrak{p}}}\right)\left(f_{\mathfrak{p}}\right)=0$ unless $\delta_{\mathfrak{p}}$ is unramified, in which case

$$
\int_{G\left(F_{v}\right)} f_{\mathfrak{p}}\left(g_{\mathfrak{p}}\right) \delta_{\mathfrak{p}}\left(N_{D_{\mathfrak{p}}}\left(x g_{\mathfrak{p}}\right)\right) d g_{\mathfrak{p}}=\delta_{\mathfrak{p}}\left(N_{D_{\mathfrak{p}}}(x)\right) \operatorname{vol}\left(Z_{\mathfrak{p}} R_{\mathfrak{p}}^{\times} \backslash R_{\mathfrak{p}}^{\times}\right) \hat{f}_{\mathfrak{p}}\left(\delta_{\mathfrak{p}} \circ N_{D_{\mathfrak{p}}}\right) .
$$


Finally, let $v$ be an archimedean place of $F$. In this case we have $F_{v} \cong \mathbf{R}$ and, under this isomorphism, $N_{D_{v}}\left(D_{v}^{\times}\right)=\mathbf{R}^{+}$. Hence since $\delta_{v}$ is quadratic $\delta_{v}\left(N_{D_{v}}\left(g_{v}\right)\right)=1$ for all $g_{v} \in D_{v}^{\times}$. Thus for $v \in \Sigma_{\infty}$,

$$
\int_{G\left(F_{v}\right)} f_{v}\left(g_{v}\right) \delta_{v}\left(N_{D_{v}}\left(x g_{v}\right)\right) d g_{v}=\int_{G\left(F_{v}\right)} \overline{\left\langle\pi_{2 k_{v}}^{\prime}(g) w_{v}, w_{v}\right\rangle} d g_{v}
$$

by definition of $f_{v}$. Clearly this integral is zero unless $\pi_{2 k_{v}}^{\prime}$ is the trivial representation which is the case if and only if $2 k_{v}=2$. Thus for $v \in \Sigma_{\infty}$,

$$
\int_{G\left(F_{v}\right)} f_{v}\left(g_{v}\right) \delta_{v}\left(N_{D_{v}}\left(x g_{v}\right)\right) d g_{v}= \begin{cases}0, & \text { if } 2 k_{v}>2 \\ \operatorname{vol}\left(G\left(F_{v}\right)\right), & \text { if } 2 k_{v}=2\end{cases}
$$

Putting these local calculations together shows that $\left(R(f)\left(\delta \circ N_{D}\right)\right)(x)$ is zero unless $\delta$ is everywhere unramified and $k_{v}=1$ for all $v \in \Sigma_{\infty}$ in which case $\left(R(f)\left(\delta \circ N_{D}\right)\right)(x)=\delta\left(N_{D}(x)\right) \hat{f}_{\mathfrak{p}}\left(\delta_{\mathfrak{p}} \circ N_{D_{\mathfrak{p}}}\right) \prod_{v<\infty} \operatorname{vol}\left(Z_{v} \backslash Z_{v} R_{v}^{\times}\right) \prod_{v \in \Sigma_{\infty}} \operatorname{vol}\left(G\left(F_{v}\right)\right)$.

Finally to finish the proof it remains to observe that

$$
K_{f, r e s}(x, y)=\sum_{\delta} \frac{\left(R(f)\left(\delta \circ N_{D}\right)\right)(x) \overline{\left(\delta \circ N_{D}\right)(y)}}{\operatorname{vol}\left(G(F) \backslash G\left(\mathbf{A}_{F}\right)\right)} .
$$

We can now compute $I_{\text {res }}(f)$.

Lemma 3.6. For $f \in C_{c}^{\infty}\left(G\left(\mathbf{A}_{F}\right)\right)$ as in Section 3.1,

$$
I_{\text {res }}(f)=C\left(\boldsymbol{k}, \Omega, f_{\mathfrak{p}}\right) \lambda(\mathfrak{N}, \boldsymbol{k}) \frac{\operatorname{vol}\left(\mathbf{A}_{F}^{\times} E^{\times} \backslash \mathbf{A}_{E}^{\times}\right)^{2}}{\operatorname{vol}\left(G(F) \backslash G\left(\mathbf{A}_{F}\right)\right)},
$$

where $C\left(\boldsymbol{k}, \Omega, f_{\mathfrak{p}}\right)=0$ unless $k_{v}=1$ for all $v \in \Sigma_{\infty}$ and $\Omega$ is of the form $\Omega=\delta \circ N_{E / F}$ for an everywhere unramified character $\delta$ of $F^{\times} \backslash \mathbf{A}_{F}^{\times}$of order at most 2. In this latter case we have

$C\left(\boldsymbol{k}, \Omega, f_{\mathfrak{p}}\right)= \begin{cases}\hat{f}_{\mathfrak{p}}\left(\delta_{\mathfrak{p}} \circ N_{D}\right)+\hat{f}_{\mathfrak{p}}\left(\eta_{\mathfrak{p}} \delta_{\mathfrak{p}} \circ N_{D}\right), & \text { if E/F is unramified everywhere; } \\ \hat{f}_{\mathfrak{p}}\left(\delta_{\mathfrak{p}} \circ N_{D}\right), & \text { otherwise. }\end{cases}$

Proof. By definition

$$
I_{r e s}(f)=\int_{E^{\times} \mathbf{A}_{F}^{\times} \backslash \mathbf{A}_{E}^{\times}} \int_{E^{\times} \mathbf{A}_{F}^{\times} \backslash \mathbf{A}_{E}^{\times}} K_{f, r e s}\left(t_{1}, t_{2}\right) \Omega\left(t_{1}^{-1} t_{2}\right) d t_{1} d t_{2} .
$$

From Lemma 3.5 we see that $I_{r e s}(f)=0$ unless $k_{v}=1$ for all $v \in \Sigma_{\infty}$, in which case,

$I_{\text {res }}(f)=\frac{\lambda(\mathfrak{N}, \boldsymbol{k})}{\operatorname{vol}\left(G(F) \backslash G\left(\mathbf{A}_{F}\right)\right)} \sum_{\delta \in X^{u n}(F)} \hat{f}_{\mathfrak{p}}\left(\delta_{\mathfrak{p}} \circ N_{D_{\mathfrak{p}}}\right)\left|\int_{E^{\times} \mathbf{A}_{F}^{\times} \backslash \mathbf{A}_{E}^{\times}} \delta\left(N_{D}(t)\right) \Omega\left(t^{-1}\right) d t\right|^{2}$. 
Considering $E^{\times}$as a subgroup of $D^{\times}$we note that $\left.N_{D}\right|_{E^{\times}}: E^{\times} \rightarrow F^{\times}$is equal to $N_{E / F}: E^{\times} \rightarrow F^{\times}$. Hence,

$$
\left|\int_{E^{\times} \mathbf{A}_{F}^{\times} \backslash \mathbf{A}_{E}^{\times}} \delta\left(N_{D}(t)\right) \Omega\left(t^{-1}\right) d t\right|^{2}= \begin{cases}\operatorname{vol}\left(E^{\times} \mathbf{A}_{F}^{\times} \backslash \mathbf{A}_{E}^{\times}\right)^{2}, & \text { if } \Omega=\delta \circ N_{E / F} ; \\ 0, & \text { otherwise. }\end{cases}
$$

Finally it suffices to note that if $\Omega=\delta \circ N_{E / F}$ with $\delta \in X^{u n}(F)$ then the only other quadratic character $\chi$ such that $\Omega=\chi \circ N_{E / F}$ is $\chi=\delta \eta$, and since $\delta$ is assumed to be unramified, $\delta \eta \in X^{u n}(F)$ if and only if $\eta$ is unramified.

We now set about computing

$$
I_{\text {cusp }}(f)=\sum_{\pi^{\prime} \in \mathcal{A}_{\text {cusp }}(G)} I_{\pi^{\prime}}(f) .
$$

It is clear from the results of Section 3.2 and Fact 3.1 that if $\pi^{\prime} \in \mathcal{A}_{\text {cusp }}(G)$ then $R(f)$ is zero on the space of $\pi^{\prime}$ unless $\pi^{\prime} \in \mathcal{F}^{\prime}(\mathfrak{N}, 2 \boldsymbol{k})$. Hence,

Lemma 3.7. For $f \in C_{c}^{\infty}\left(G\left(\mathbf{A}_{F}\right)\right)$ as in Section 3.1,

$$
I_{\text {cusp }}(f)=\sum_{\pi^{\prime} \in \mathcal{F}^{\prime}(\mathfrak{N}, 2 k)} I_{\pi^{\prime}}(f) .
$$

It remains to compute $I_{\pi^{\prime}}(f)$ for $\pi^{\prime} \in \mathcal{F}^{\prime}(\mathfrak{N}, 2 \boldsymbol{k})$.

Lemma 3.8. For $f \in C_{c}^{\infty}\left(G\left(\mathbf{A}_{F}\right)\right)$ as in Section 3.1 and $\pi^{\prime} \in \mathcal{F}^{\prime}(\mathfrak{N}, 2 \boldsymbol{k})$,

$I_{\pi^{\prime}}(f)=\lambda(\mathfrak{N}, \boldsymbol{k}) \frac{L\left(1 / 2, \pi_{E} \otimes \Omega\right)}{L(1, \pi, A d)} \frac{L\left(2,1_{F}\right) L_{S(\Omega)}(1, \eta)^{2} \sqrt{\left|\Delta_{F}\right|}}{2 \sqrt{c(\Omega)\left|\Delta_{E}\right|}} \prod_{v \mid \mathfrak{N}}\left(1-q_{v}^{-1}\right) \prod_{v \in \Sigma_{\infty}} \frac{\Gamma\left(2 k_{v}\right)}{\pi \Gamma\left(k_{v}+m_{v}\right) \Gamma\left(k_{v}-m_{v}\right)}$,

where $\pi=\mathrm{JL}\left(\pi^{\prime}\right)$.

Proof. Let $\pi^{\prime} \in \mathcal{F}^{\prime}(\mathfrak{N}, 2 \boldsymbol{k})$ and let $V_{\pi^{\prime}}$ denote the space of $\pi^{\prime}$. Let $W_{\pi^{\prime}}$ denote the space of $w \in V_{\pi^{\prime}}$ such that,

1. $\pi^{\prime}(k) w=w$ for all $k \in \prod_{v<\infty} R_{v}^{\times}$, and

2. $\pi^{\prime}(t) w=\Omega(t) w$ for all $t \in E_{v}^{\times}, v \in \Sigma_{\infty}$.

By the results of Section $3.2 W_{\pi^{\prime}}$ is one dimensional. We fix a non-zero element $\varphi_{\pi^{\prime}} \in W_{\pi^{\prime}}$. By our choice of $f$ it again follows from the results of Section 3.2 that $R(f)$ kills the orthogonal complement of $\varphi_{\pi^{\prime}}$ in $\pi^{\prime}$ and

$$
R(f) \varphi_{\pi^{\prime}}=\lambda(\mathfrak{N}, \boldsymbol{k}) \hat{f}_{\mathfrak{p}}\left(\pi_{\mathfrak{p}}\right) \varphi_{\pi^{\prime}} .
$$

Hence,

$$
I_{\pi^{\prime}}(f)=\lambda(\mathfrak{N}, \boldsymbol{k}) \hat{f}_{\mathfrak{p}}\left(\pi_{\mathfrak{p}}\right) \frac{\left|\int_{\mathbf{A}_{F}^{\times} E^{\times} \backslash \mathbf{A}_{E}^{\times}} \varphi_{\pi^{\prime}}(t) \Omega^{-1}(t) d t\right|^{2}}{\left(\varphi_{\pi^{\prime}}, \varphi_{\pi^{\prime}}\right)} .
$$


We can now apply [MW09, Theorem 4.1] which gives, for $\pi^{\prime} \in \mathcal{F}^{\prime}(\mathfrak{N}, 2 \boldsymbol{k})$, an equality between

$$
\frac{\left|\int_{\mathbf{A}_{F}^{\times} E^{\times} \backslash \mathbf{A}_{E}^{\times}} \varphi_{\pi^{\prime}}(t) \Omega^{-1}(t) d t\right|^{2}}{\left(\varphi_{\pi^{\prime}}, \varphi_{\pi^{\prime}}\right)}
$$

and

$$
\frac{L\left(1 / 2, \pi_{E} \otimes \Omega\right)}{L(1, \pi, A d)} \frac{L\left(2,1_{F}\right) L_{S(\Omega)}(1, \eta)^{2} \sqrt{\left|\Delta_{F}\right|}}{2 \sqrt{c(\Omega)\left|\Delta_{E}\right|}} \prod_{v \mid \mathfrak{N}}\left(1-q_{v}^{-1}\right) \prod_{v \in \Sigma_{\infty}} \frac{\Gamma\left(2 k_{v}\right)}{\pi \Gamma\left(k_{v}+m_{v}\right) \Gamma\left(k_{v}-m_{v}\right)},
$$

where $S(\Omega)$ is the set of places of $F$ above which $\Omega$ is ramified and $L(s, \pi, A d)$ is the adjoint $L$-function of $\pi$. For the ease of the reader we note that for $\pi=\operatorname{JL}\left(\pi^{\prime}\right) \in \mathcal{F}(\mathfrak{N}, 2 \boldsymbol{k})$ we have, in the notation of [MW09, Theorem 4.1], $S^{\prime}(\pi)=\emptyset$; at the places $v \in \operatorname{Ram}(\pi), e_{v}(E / F)=1$; and for $v \in \Sigma_{\infty}, C_{v}(E, \pi, \Omega)$ is equal to the quotient of gamma functions appearing above. The $L\left(2,1_{F}\right)$ term appears in the formula here, and not in [MW09, Theorem 4.1], due to a difference in the choice of measures on $G\left(\mathbf{A}_{F}\right)$.

We recall that

$$
\operatorname{vol}\left(G(F) \backslash G\left(\mathbf{A}_{F}\right)\right)=2,
$$

and

$$
\operatorname{vol}\left(\mathbf{A}_{F}^{\times} E^{\times} \backslash \mathbf{A}_{E}^{\times}\right)=2 L(1, \eta),
$$

and that we defined

$$
\lambda(\mathfrak{N}, \boldsymbol{k})=\frac{1}{\left|\Delta_{F}\right|^{\frac{3}{2}} L\left(2,1_{F}\right)} \prod_{v \mid \mathfrak{N}} \frac{1}{q_{v}-1} \prod_{v \in \Sigma_{\infty}} \frac{4 \pi}{2 k_{v}-1} .
$$

Now by combining our calculation of $I_{\text {res }}(f)$ from Lemma 3.6 with our calculation of $I_{\text {cusp }}(f)$ from Lemmas 3.7 and 3.8 we obtain the following.

Proposition 3.9. For $f$ as in Section 3.1, $I(f)$ is equal to the sum of

$$
\frac{L_{S(\Omega)}(1, \eta)^{2}}{2\left|\Delta_{F}\right|^{2} \sqrt{c(\Omega) d_{E / F}}} \frac{4^{[F: \mathbf{Q}]}}{|\mathfrak{N}|}\left(\begin{array}{c}
2 \boldsymbol{k}-2 \\
\boldsymbol{k}+\boldsymbol{m}-1
\end{array}\right) \sum_{\pi \in \mathcal{F}(\mathfrak{N}, 2 \boldsymbol{k})} \frac{L\left(1 / 2, \pi_{E} \otimes \Omega\right)}{L(1, \pi, A d)} \hat{f}_{\mathfrak{p}}\left(\pi_{\mathfrak{p}}\right),
$$

and

$$
C\left(\boldsymbol{k}, \Omega, f_{\mathfrak{p}}\right) \frac{2 L(1, \eta)^{2}}{\left|\Delta_{F}\right|^{\frac{3}{2}} L\left(2,1_{F}\right)} \prod_{v \mid \mathfrak{N}} \frac{1}{q_{v}-1} \prod_{v \in \Sigma_{\infty}} \frac{4 \pi}{2 k_{v}-1},
$$

where $C\left(\boldsymbol{k}, \Omega, f_{\mathfrak{p}}\right)$ is defined in Lemma 3.6. 


\section{Geometric side of the trace formula}

Recall that

$$
I(f)=\int_{\mathbf{A}_{F}^{\times} E^{\times} \backslash \mathbf{A}_{E}^{\times}} \int_{\mathbf{A}_{F}^{\times} E^{\times} \backslash \mathbf{A}_{E}^{\times}} K_{f}\left(t_{1}, t_{2}\right) \Omega\left(t_{1}^{-1} t_{2}\right) d t_{1} d t_{2} .
$$

A quick calculation shows that the following matrices are a set of representatives for the double cosets of $E^{\times} \backslash D^{\times} / E^{\times}$:

$$
\left\{\left(\begin{array}{ll}
1 & 0 \\
0 & 1
\end{array}\right),\left(\begin{array}{ll}
0 & \epsilon \\
1 & 0
\end{array}\right)\right\} \cup\left\{\left(\begin{array}{cc}
1 & \epsilon x \\
\bar{x} & 1
\end{array}\right): x \in E^{\times} / E^{1}\right\} .
$$

The first two cosets are referred to as irregular cosets and the remaining cosets are called regular cosets.

By the geometric expansion of the relative trace formula [JC01, Section 1 $(8)]$,

$$
I(f)=\sum_{\xi \in \epsilon N E^{\times}} I(\xi, f)+\operatorname{vol}\left(\mathbf{A}_{F}^{\times} E^{\times} \backslash \mathbf{A}_{E}^{\times}\right)\left[I(0, f)+\delta\left(\Omega^{2}\right) I(\infty, f)\right]
$$

where for an idele class character $\chi, \delta(\chi)=1$ if $\chi=1$ and $\delta(\chi)=0$ otherwise. For $\xi=\epsilon x \bar{x}$,

$$
\begin{gathered}
I(\xi, f):=\int_{\mathbf{A}_{F}^{\times} \backslash \mathbf{A}_{E}^{\times}} \int_{\mathbf{A}_{F}^{\times} \backslash \mathbf{A}_{E}^{\times}} f\left(t_{1}\left(\begin{array}{cc}
1 & \epsilon x \\
\bar{x} & 1
\end{array}\right) t_{2}\right) \Omega\left(t_{1} t_{2}\right) d t_{1} d t_{2}, \\
I(0, f):=\int_{\mathbf{A}_{F}^{\times} \backslash \mathbf{A}_{E}^{\times}} f(t) \Omega(t) d t,
\end{gathered}
$$

and

$$
I(\infty, f):=\int_{\mathbf{A}_{F}^{\times} \backslash \mathbf{A}_{E}^{\times}} f\left(t\left(\begin{array}{ll}
0 & \epsilon \\
1 & 0
\end{array}\right)\right) \Omega(t) d t .
$$

We remark that these integrals factor as a product of local ones, which we denote with similar notation. We note that the geometric expansion in [JC01] has $\delta\left(\Omega^{2}\right)$ next to $I(0, f)$ rather than $I(\infty, f)$. This is because $K_{f}\left(t_{1}, t_{2}\right)$ is integrated against $\Omega\left(t_{1} t_{2}\right)^{-1}$ rather than against $\Omega\left(t_{1}^{-1} t_{2}\right)$.

In this section we compute the period integrals $I(g, f)$ for $f$ chosen as in Section 3.1. First we make the necessary local calculations in Sections 4.1 and 4.2 and then we bring together the local calculations to write down the geometric side of the global relative trace formula in Section 4.3. We note that the choice of local function $f_{v}$ is intrinsic to $G\left(F_{v}\right)$, as is the parameterization of the double cosets. Hence for simplicity we can fix the following identifications for the remainder of this section.

For $v \notin \Sigma_{\infty}$,

$$
\begin{array}{r}
D_{v}=\left\{\left(\begin{array}{cc}
\alpha & \epsilon_{v} \beta \\
\bar{\beta} & \bar{\alpha}
\end{array}\right)\right\}, E_{v}=\left\{\left(\begin{array}{cc}
\alpha & 0 \\
0 & \bar{\alpha}
\end{array}\right)\right\}, \text { where } \epsilon_{v}=1 \text { if } v \nmid \mathfrak{N}, \\
\epsilon_{v}=\varpi_{v} \text { if } v \mid \mathfrak{N}
\end{array}
$$


and for $v \in \Sigma_{\infty}$,

$$
D_{v}=\left\{\left(\begin{array}{cc}
\alpha & -\beta \\
\bar{\beta} & \bar{\alpha}
\end{array}\right)\right\}, E_{v}=\left\{\left(\begin{array}{cc}
\alpha & 0 \\
0 & \bar{\alpha}
\end{array}\right)\right\} .
$$

For $v$ that splits in $E$, let $E_{v}=F_{v} \oplus F_{v}$. For $v \notin \Sigma_{\infty}$, let $\tau_{v}$ be such that $\mathcal{O}_{E_{v}}=\mathcal{O}_{F_{v}}\left[\tau_{v}\right]$ and $\tau_{v}$ is a uniformizer in $E_{v}$ if $E_{v} / F_{v}$ is ramified. If $v$ is inert in $E$, let $\varpi_{E_{v}}=\varpi_{v}$. If $v$ ramifies, let $\varpi_{E_{v}}=\tau_{v}$. If $v$ splits let $\varpi_{E_{v}} \in \mathcal{O}_{E_{v}}$ be such that $v\left(N\left(\varpi_{E_{v}}\right)\right)=1$. For a valuation $v$ of $F$ that is not split in $E$, let $v_{E}$ be the corresponding valuation on $E$. Let

$R_{v}=\left\{\left(\begin{array}{cc}\alpha & \epsilon_{v} \beta \\ \bar{\beta} & \bar{\alpha}\end{array}\right): \alpha \in \frac{1}{\left(\bar{\tau}_{v}-\tau_{v}\right) \varpi_{v}^{n\left(\Omega_{v}\right)}}\left(\mathcal{O}_{F_{v}}+\varpi_{v}^{n\left(\Omega_{v}\right)} \mathcal{O}_{E_{v}}\right), \alpha+\beta \in \mathcal{O}_{F_{v}}+\varpi_{v}^{n\left(\Omega_{v}\right)} \mathcal{O}_{E_{v}}\right\}$.

This is a maximal order in $D_{v}$ such that $R_{v} \cap E_{v}=\mathcal{O}_{F_{v}}+\varpi_{v}^{n\left(\Omega_{v}\right)} \mathcal{O}_{E_{v}}$.

For the rest of this section we will drop the $v$ from our notation and let $n=n\left(\Omega_{v}\right)$ when it is clear that we are working locally.

\subsection{Irregular cosets}

First we compute the orbital integrals associated to the irregular cosets.

Lemma 4.1. Let $v$ divide $\mathfrak{N}$. Then

$$
I\left(0, f_{v}\right)=\operatorname{vol}\left(F_{v}^{\times} \backslash F_{v}^{\times}\left(1+\varpi_{v}^{n\left(\Omega_{v}\right)} \mathcal{O}_{E_{v}}\right)\right)^{\times} .
$$

Proof. We have

$$
\begin{aligned}
I\left(0, f_{v}\right) & =\int_{F_{v}^{\times} \backslash E_{v}^{\times}} f_{v}\left(\left(\begin{array}{cc}
a & 0 \\
0 & \bar{a}
\end{array}\right)\right) \Omega_{v}(a) d^{\times} a \\
& =\operatorname{vol}\left(F_{v}^{\times} \backslash F_{v}^{\times}\left(1+\varpi_{v}^{n\left(\Omega_{v}\right)} \mathcal{O}_{E_{v}}\right)\right)^{\times} .
\end{aligned}
$$

Lemma 4.2. Let $v$ divide $\mathfrak{N}$. Then

$$
I\left(\infty, f_{v}\right)=0 .
$$

Proof.

$$
I\left(\infty, f_{v}\right)=\int_{F_{v}^{\times} \backslash E_{v}^{\times}} f_{v}\left(\left(\begin{array}{cc}
0 & \varpi_{v} a \\
\bar{a} & 0
\end{array}\right)\right) \Omega_{v}(a) d^{\times} a .
$$

Clearly

$$
\left(\begin{array}{cc}
0 & \varpi_{v} a \\
\bar{a} & 0
\end{array}\right) \notin Z_{v} R_{v}^{\times}
$$

because $Z_{v} R_{v}^{\times}$only contains matrices, $g$, where $v(\operatorname{det} g)$ is even.

Before we compute the irregular orbital integrals for finite $v$ away from $\mathfrak{N}$, we need the following technical lemma. 
Lemma 4.3. Let $v$ be a finite prime not dividing $\mathfrak{N}$. Let $\alpha=a_{\alpha}+b_{\alpha} \tau_{v}$ and $\beta=a_{\beta}+b_{\beta} \tau_{v}$. Then $F_{v}^{\times} R_{v}^{\times} \alpha R_{v}^{\times}=F_{v}^{\times} R_{v}^{\times} \beta R_{v}^{\times}$if and only if $v(N(\alpha))-2 \min \left\{v\left(a_{\alpha}\right), v\left(\varpi_{v}^{-n\left(\Omega_{v}\right)} b_{\alpha}\right)\right\}=v(N(\beta))-2 \min \left\{v\left(a_{\beta}\right), v\left(\varpi_{v}^{-n\left(\Omega_{v}\right)} b_{\beta}\right)\right\}$.

Proof. For the proof we can take $D=M(2, F)$ and embed $E$ as

$$
E \hookrightarrow M(2, F): a+b \tau \mapsto\left(\begin{array}{cc}
a+b \operatorname{Tr}_{E / F}(\tau) & b \varpi^{-n} \\
-b \varpi^{n} N(\tau) & a
\end{array}\right) .
$$

We set $R=M\left(2, \mathcal{O}_{F}\right)$, then we have $R \cap E=\mathcal{O}_{F}+\varpi^{n} \mathcal{O}_{E}$. We recall that

$$
\mathrm{GL}(2, F)=\bigsqcup_{m \geq 0} F^{\times} R^{\times}\left(\begin{array}{ll}
\varpi^{m} & \\
& 1
\end{array}\right) R^{\times},
$$

and

$$
g=\left(\begin{array}{ll}
a & b \\
c & d
\end{array}\right) \in F^{\times} R^{\times}\left(\begin{array}{ll}
\varpi^{m} & \\
& 1
\end{array}\right) R^{\times},
$$

if and only if

$$
m=v(\operatorname{det} g)-2 \min \{v(a), v(b), v(c), v(d)\} .
$$

Thus we see that

$$
\alpha=a+b \tau \in F^{\times} R^{\times}\left(\begin{array}{cc}
\varpi^{m} & \\
& 1
\end{array}\right) R^{\times},
$$

if and only if

$$
m=v(N(\alpha))-2 \min \left\{v(a), v\left(\varpi^{-n} b\right)\right\} .
$$

For $f_{v} \in \mathcal{H}\left(G\left(F_{v}\right), Z_{v} R_{v}^{\times}\right)$we have

$$
\begin{aligned}
I\left(0, f_{v}\right) & =\int_{F_{v}^{\times} \backslash E_{v}^{\times}} f_{v}(a) \Omega_{v}(a) d^{\times} a \\
& =\operatorname{vol}\left(F_{v}^{\times} \backslash F_{v}^{\times}\left(\mathcal{O}_{F_{v}}+\varpi_{v}^{n\left(\Omega_{v}\right)} \mathcal{O}_{E_{v}}\right)^{\times}\right) \tilde{I}\left(f_{v}\right),
\end{aligned}
$$

where

$$
\tilde{I}\left(f_{v}\right):=\sum_{\alpha \in F_{v}^{\times}\left(\mathcal{O}_{F_{v}}+\varpi_{v}^{n\left(\Omega_{v}\right)} \mathcal{O}_{E_{v}}\right) \times \backslash E_{v}^{\times}} f_{v}(\alpha) \Omega_{v}(\alpha) .
$$

For finite $v$ away from $\mathfrak{N},\left(\begin{array}{ll}0 & \epsilon \\ 1 & 0\end{array}\right) \in R_{v}^{\times}$, and hence

$$
\begin{aligned}
I\left(\infty, f_{v}\right) & =\int_{F_{v}^{\times} \backslash E_{v}^{\times}} f_{v}\left(a\left(\begin{array}{cc}
0 & \epsilon_{v} \\
1 & 0
\end{array}\right)\right) \Omega_{v}(a) d^{\times} a \\
& =\int_{F_{v}^{\times} \backslash E_{v}^{\times}} f_{v}(a) \Omega_{v}(a) d^{\times} a \\
& =I\left(0, f_{v}\right) .
\end{aligned}
$$

In the following three lemmas, we compute $\tilde{I}\left(f_{v}\right)$ for $v$ inert, ramified and split in $E$. 
Lemma 4.4. Let $v$ be a finite prime not dividing $\mathfrak{N}$, which is inert in $E$. For $f_{v} \in \mathcal{H}\left(G\left(F_{v}\right), Z_{v} R_{v}^{\times}\right)$,

$$
\tilde{I}\left(f_{v}\right)= \begin{cases}f_{v}(1) & \text { when } n\left(\Omega_{v}\right)=0 \\ f_{v}(1)-f_{v}\left(1+\tau_{v} \varpi_{v}^{n\left(\Omega_{v}\right)-1}\right) & \text { when } n\left(\Omega_{v}\right)>0 .\end{cases}
$$

Proof. Recall that $n=n\left(\Omega_{v}\right)$. We note that a set of representatives for $F^{\times}\left(\mathcal{O}_{F}+\right.$ $\left.\varpi^{n} \mathcal{O}_{E}\right)^{\times} \backslash E^{\times}$is given by

$$
\left\{1+b \tau: b \in \mathcal{O}_{F} / \mathfrak{p}_{F}^{n}\right\} \cup\left\{a+\tau: a \in \mathfrak{p}_{F} / \mathfrak{p}_{F}^{n}\right\} .
$$

Applying Lemma 4.3 we see that

$$
\tilde{I}(f)=\sum_{k=0}^{n} f\left(1+\tau \varpi^{k}\right) \sum_{b \in\left(\mathfrak{p}_{F}^{k} / \mathfrak{p}_{F}^{n}\right)^{\times}} \Omega(1+b \tau)+f(\tau) \sum_{a \in \mathfrak{p}_{F} / \mathfrak{p}_{F}^{n}} \Omega(a+\tau) .
$$

The lemma follows by the observation that

$$
\sum_{b \in\left(\mathfrak{p}_{F}^{k} / \mathfrak{p}_{F}^{n}\right) \times} \Omega(1+b \tau)= \begin{cases}0, & \text { if } 0 \leq k \leq n-1 \\ -1, & \text { if } k=n-1 \\ 1, & \text { if } k=n\end{cases}
$$

and that

$$
\sum_{a \in \mathfrak{p}_{F} / \mathfrak{p}_{F}^{n}} \Omega(a+\tau)=0
$$

Lemma 4.5. Let $v$ be a finite prime which is ramified in $E$. For $f_{v} \in \mathcal{H}\left(G\left(F_{v}\right), Z_{v} R_{v}^{\times}\right)$,

$$
\tilde{I}\left(f_{v}\right)= \begin{cases}f_{v}(1)+\Omega_{v}\left(\tau_{v}\right) f_{v}\left(\tau_{v}\right) & \text { when } n\left(\Omega_{v}\right)=0 \\ f_{v}(1)-f_{v}\left(1+\tau_{v} \varpi_{v}^{n\left(\Omega_{v}\right)-1}\right) & \text { when } n\left(\Omega_{v}\right)>0 .\end{cases}
$$

Proof. We note that a set of representatives for $F^{\times}\left(\mathcal{O}_{F}+\varpi^{n} \mathcal{O}_{E}\right)^{\times} \backslash E^{\times}$is given by

$$
\left\{1+b \tau: b \in \mathcal{O}_{F} / \mathfrak{p}_{F}^{n}\right\} \cup\left\{a \varpi+\tau: a \in \mathcal{O}_{F} / \mathfrak{p}_{F}^{n}\right\} .
$$

Applying Lemma 4.3 we see that

$$
\tilde{I}(f)=\sum_{k=0}^{n} f\left(1+\tau \varpi^{k}\right) \sum_{b \in\left(\mathfrak{p}_{F}^{k} / \mathfrak{p}_{F}^{n}\right)^{\times}} \Omega(1+b \tau)+f(\varpi+\tau) \sum_{a \in \mathcal{O}_{F} / \mathfrak{p}_{F}^{n}} \Omega(a \varpi+\tau) .
$$

The lemma follows by the observation that

$$
\sum_{b \in\left(\mathfrak{p}_{F}^{k} / \mathfrak{p}_{F}^{n}\right) \times} \Omega(1+b \tau)= \begin{cases}0, & \text { if } 0 \leq k \leq n-1 \\ -1, & \text { if } k=n-1 \\ 1, & \text { if } k=n\end{cases}
$$


And that

$$
\sum_{a \in \mathcal{O}_{F} / \mathfrak{p}_{F}^{n}} \Omega(a \varpi+\tau)= \begin{cases}\Omega(\tau), & \text { if } n=0 \\ 0, & \text { if } n>0\end{cases}
$$

Lemma 4.6. Let $v$ be a finite prime which is split in $E$. Let $f_{v} \in \mathcal{H}\left(G\left(F_{v}\right), R_{v}^{\times}\right)$. When $n\left(\Omega_{v}\right)=0$ we have

$$
\tilde{I}\left(f_{v}\right)=\sum_{\alpha \in E_{v}^{\times} / F_{v}^{\times} \mathcal{O}_{E_{v}}^{\times}} \Omega_{v}(\alpha) f_{v}(\alpha),
$$

and when $n\left(\Omega_{v}\right)>0$ we have

$$
\tilde{I}\left(f_{v}\right)=f_{v}(1)-f_{v}\left(1+\tau_{v} \varpi_{v}^{n\left(\Omega_{v}\right)-1}\right) .
$$

Proof. In this case we take $R=M\left(2, \mathcal{O}_{F}\right)$ and embed $E$ as

$$
(a, b) \mapsto\left(\begin{array}{cc}
a & \varpi^{-n}(a-b) \\
0 & b
\end{array}\right) .
$$

By Lemma 4.3 we have

$$
(a, b) \in F^{\times} R^{\times}\left(\begin{array}{cc}
\varpi^{m} & 0 \\
0 & 1
\end{array}\right) R^{\times}
$$

if and only if $m=v(a b)-2 \min \left\{v(a), v\left(\varpi^{-n}(a-b)\right)\right\}$. We recall

$$
\begin{aligned}
\tilde{I}(f) & =\sum_{\alpha \in F^{\times}\left(\mathcal{O}_{F}+\varpi^{n(\Omega)} \mathcal{O}_{E}\right)^{\times} \backslash E^{\times}} f(\alpha) \Omega(\alpha) \\
& =\sum_{\alpha \in U_{F}^{n} \backslash F^{\times}} \Omega(\alpha, 1) f(\alpha, 1) .
\end{aligned}
$$

Thus we see that if $n=0$ then

$$
\tilde{I}(f)=f(1)+\sum_{m=1}^{\infty}\left(\Omega\left(\varpi^{m}, 1\right)+\Omega\left(\varpi^{-m}, 1\right)\right) f\left(\varpi^{m}, 1\right) .
$$

On the other hand if $n>0$, then only $\alpha \in U_{F}$ contribute to the sum, thus

$$
\begin{aligned}
\tilde{I}(f) & =\sum_{a \in U_{F}^{n} \backslash U_{F}} \Omega(a, 1) f(a, 1) \\
& =f(1,1)-f\left(1+\varpi^{n-1}, 1\right) .
\end{aligned}
$$

We now compute the irregular orbital integrals at the archimedean places.

Lemma 4.7. Let $v \in \Sigma_{\infty}$. Then $I\left(0, f_{v}\right)=\operatorname{vol}\left(F_{v}^{\times} \backslash E_{v}^{\times}\right)$. 
Proof. We have

$$
\begin{aligned}
I\left(0, f_{v}\right) & =\int_{F_{v}^{\times} \backslash E_{v}^{\times}} f_{v}\left(\left(\begin{array}{cc}
a & 0 \\
0 & \bar{a}
\end{array}\right)\right) \Omega_{v}(a) d^{\times} a \\
& =\operatorname{vol}\left(F_{v}^{\times} \backslash E_{v}^{\times}\right) .
\end{aligned}
$$

Lemma 4.8. Let $v \in \Sigma_{\infty}$. When $\alpha \neq 0$ we have

$$
\begin{aligned}
& f_{v}\left(\begin{array}{cc}
\alpha & -\beta \\
\bar{\beta} & \bar{\alpha}
\end{array}\right) \\
= & \frac{1}{(\alpha \bar{\alpha}+\beta \bar{\beta})^{k_{v}-1}} \sum_{i=0}^{k_{v}-\left|m_{v}\right|-1}(-1)^{i}\left(\begin{array}{c}
k_{v}+m_{v}-1 \\
i
\end{array}\right)\left(\begin{array}{c}
k_{v}-m_{v}-1 \\
i
\end{array}\right)(\beta \bar{\beta})^{i}(\alpha \bar{\alpha})^{k_{v}-1-i}\left(\frac{\alpha}{\bar{\alpha}}\right)^{-m_{v}},
\end{aligned}
$$

when $m_{v}=0$ we have

$$
f_{v}\left(\begin{array}{cc}
0 & -\beta \\
\bar{\beta} & 0
\end{array}\right)=(-1)^{k_{v}-1}
$$

and when $m_{v} \neq 0$ we have

$$
f_{v}\left(\begin{array}{cc}
0 & -\beta \\
\bar{\beta} & 0
\end{array}\right)=0 .
$$

Proof. Let $\pi$ be the discrete series representation of $\operatorname{PGL}(2, \mathbf{R})$ of weight $2 k$. Recall that $\Omega$ is the character of $\mathbf{C}^{\times}$given by

$$
\Omega: z \mapsto\left(\frac{z}{\bar{z}}\right)^{m}
$$

where $m$ is an integer with $|m|<k$. We have

$$
D^{\times}(\mathbf{R})=\left\{\left(\begin{array}{cc}
\alpha & -\beta \\
\bar{\beta} & \bar{\alpha}
\end{array}\right) \in \mathrm{GL}(2, \mathbf{C})\right\} .
$$

Viewing $D^{\times}(\mathbf{R}) \subset \mathrm{GL}(2, \mathbf{C})$ gives an irreducible 2-dimensional representation $V$ of $D^{\times}(\mathbf{R})$. We take $\pi^{\prime}$ to be the representation of $G(\mathbf{R})$ which corresponds to $\pi$ via the Jacquet-Langlands correspondence. Thus

$$
\pi^{\prime}=\operatorname{Sym}^{2 k-2} V \otimes \operatorname{det}^{-(k-1)} .
$$

We realize $\pi^{\prime}$ on the space of homogeneous polynomials in $x$ and $y$ of degree $2 k-2$, with $g$ acting by

$$
\pi^{\prime}(g): x \mapsto \alpha x+\bar{\beta} y, y \mapsto-\beta x+\bar{\alpha} y .
$$

We set, for $0 \leq i \leq 2 k-2$,

$$
v_{i}=x^{i} y^{2 k-2-i},
$$


so that

$$
\pi^{\prime}\left(\begin{array}{cc}
\alpha & 0 \\
0 & \bar{\alpha}
\end{array}\right) v_{i}=\left(\frac{\alpha}{\bar{\alpha}}\right)^{i-(k-1)} v_{i}
$$

Hence we have

$$
\pi^{\prime}\left(\begin{array}{cc}
\alpha & 0 \\
0 & \bar{\alpha}
\end{array}\right) v_{m+k-1}=\Omega(\alpha) v_{m+k-1} .
$$

We have, for $g \in G(\mathbf{R})$,

$$
f(g)=\frac{\overline{\left\langle\pi^{\prime}(g) v_{m+k-1}, v_{m+k-1}\right\rangle}}{\left\langle v_{m+k-1}, v_{m+k-1}\right\rangle} .
$$

We compute that

$$
\begin{aligned}
& f\left(\begin{array}{cc}
\alpha & -\beta \\
\bar{\beta} & \bar{\alpha}
\end{array}\right) \\
= & \frac{1}{(\alpha \bar{\alpha}+\beta \bar{\beta})^{k-1}} \sum_{i=0}^{k-|m|-1}(-1)^{i}\left(\begin{array}{c}
k+m-1 \\
i
\end{array}\right)\left(\begin{array}{c}
k-m-1 \\
i
\end{array}\right)(\beta \bar{\beta})^{i} \bar{\alpha}^{m+k-1-i} \alpha^{k-m-1-i}
\end{aligned}
$$

and the lemma now follows.

From the definition of $I\left(\infty, f_{v}\right)$ we have,

Corollary 4.9. For $v \in \Sigma_{\infty}$,

$$
I\left(\infty, f_{v}\right)= \begin{cases}\operatorname{vol}\left(F_{v}^{\times} \backslash E_{v}^{\times}\right)(-1)^{k_{v}-1}, & \text { if } m_{v}=0 ; \\ 0, & \text { otherwise. }\end{cases}
$$

\subsection{Regular cosets}

Recall that

$$
I\left(\xi, f_{v}\right)=\int_{\left(F_{v}^{\times} \backslash E_{v}^{\times}\right)^{2}} f_{v}\left(\left(\begin{array}{cc}
a b & a \bar{b} x \epsilon_{v} \\
\bar{a} b \bar{x} & \overline{a b}
\end{array}\right)\right) \Omega_{v}(a b) d^{\times} b d^{\times} a .
$$

By a change of variables this equals

$$
\int_{F_{v}^{\times} \backslash E_{v}^{\times}} \int_{E_{v}^{1}} f_{v}\left(\left(\begin{array}{cc}
a & 0 \\
0 & \bar{a}
\end{array}\right) \gamma_{x z}\right) \Omega_{v}(a) d^{\times} z d^{\times} a
$$

where $\gamma_{x z}=\left(\begin{array}{cc}1 & x z \epsilon_{v} \\ x z & 1\end{array}\right)$. For $x \in E_{v}^{\times}$, we let

$$
I\left(x, E_{v}^{1}\right):=\left\{z \in E_{v}^{1}: \exists a \in E_{v}^{\times} \text {such that } a \gamma_{x z} \in R_{v}^{\times}\right\} .
$$

In this section we compute $I\left(\xi, f_{v}\right)$ under certain simplifying assumptions, and provide a bound on $\left|I\left(\xi, f_{v}\right)\right|$ in all cases. 


\subsubsection{Exact calculations}

We begin by calculating $I\left(\xi, f_{v}\right)$ when $v$ divides $\mathfrak{N}$.

Lemma 4.10. Let $v$ divide $\mathfrak{N}$. Then

$$
I\left(\xi, f_{v}\right)= \begin{cases}0 & \text { if } v(\xi) \leq 0 \\ \operatorname{vol}\left(F_{v}^{\times} \backslash E_{v}^{\times}\right)^{2} & \text { if } v(\xi) \geq 1 .\end{cases}
$$

Proof. It is easy to see that $a \gamma_{x z} \in F^{\times} R^{\times}$exactly when $v(x) \geq 0$. Hence $v(\xi)$ must be greater than or equal to one.

We now establish a vanishing result for $I\left(\xi, f_{v}\right)$ for finite $v$ away from $\mathfrak{N}$.

Lemma 4.11. Let $v$ be a finite prime not dividing $\mathfrak{N}$. Suppose that $f_{v}$ is the characteristic function of the double coset $F_{v}^{\times} R_{v}^{\times} \gamma R_{v}^{\times}$for $\gamma \in R_{v}$. If $v(1-\xi)>$ $v\left(\mathfrak{d}_{E / F} \mathfrak{c}(\Omega) \operatorname{det}(\gamma)\right)$, then $I\left(\xi, f_{v}\right)=0$.

Proof. Clearly $a \gamma_{x z} \in F_{v}^{\times} R_{v}^{\times} \gamma R_{v}^{\times}$if and only if there exists a $\lambda \in F_{v}^{\times}$such that $\lambda a \gamma_{x z} \in R_{v}^{\times} \gamma R_{v}^{\times}$. If this matrix lies in $R_{v}^{\times} \gamma R_{v}^{\times}$, then $v\left(\lambda^{2} a \bar{a}(1-\xi)\right)=v(\operatorname{det}(\gamma))$ and $\lambda a \in \frac{1}{(\bar{\tau}-\tau) \varpi_{v}^{n}} \mathcal{O}_{E}$. Thus the orbital integral is zero unless $v(1-\xi) \leq$ $v\left(\mathfrak{d}_{E / F} \mathfrak{c}(\Omega) \operatorname{det}(\gamma)\right)$.

In the next two lemmas we compute $I\left(\xi, \mathbf{1}_{v}\right)$ when $\Omega_{v}$ is unramified.

Lemma 4.12. Let $v$ be a finite prime not dividing $\mathfrak{N}$, not split in $E$ and such that $n\left(\Omega_{v}\right)=0$. If $v$ ramifies in $E$, assume that the characteristic of the residue field of $F_{v}$ is odd. Then $I\left(\xi, \mathbf{1}_{v}\right)$ equals

$$
\operatorname{vol}\left(F_{v}^{\times} \backslash F_{v}^{\times} U_{E_{v}}\right) \operatorname{vol}\left(F_{v}^{\times} \backslash E_{v}^{\times}\right) \Omega_{v}\left(\varpi_{E_{v}}^{\frac{v_{E}(1-\xi)}{2}}\right) \times \begin{cases}0 & \text { if } v(1-\xi)>v\left(\mathfrak{d}_{E / F}\right) \\ 1 & \text { if } v(1-\xi) \leq 0 \\ \frac{1}{2} & \text { if } v(1-\xi)=v\left(\mathfrak{d}_{E / F}\right)>0 .\end{cases}
$$

Proof. We look at our orbital integral,

$$
I(\xi, \mathbf{1})=\int_{F^{\times} \backslash E^{\times}} \int_{E^{1}} \mathbf{1}\left(a \gamma_{x z}\right) \Omega(a) d^{\times} z d^{\times} a .
$$

By the definition of $R^{\times}, a \gamma_{x z}$ lies in $F^{\times} R^{\times}$if and only if there is a $\lambda \in F^{\times}$such that

1. $v\left(\lambda^{2} a \bar{a}\right)=-v(1-\xi)$

2. $\lambda a \in \frac{1}{(\bar{\tau}-\tau)} \mathcal{O}_{E}$

3. $\lambda a(1+z x) \in \mathcal{O}_{E}$. 
If $v(1-\xi) \leq v\left(\mathfrak{d}_{E / F}\right)$, then

$$
\begin{aligned}
I(\xi, \mathbf{1}) & =\int_{\left\{z \in E^{1}: 1+x z \in \varpi_{E}^{\frac{v_{E}(1-\xi)}{2}}\right.} \mathcal{O}_{E\}} \int_{F^{\times} \backslash F^{\times} \varpi_{E}^{\frac{-v_{E}(1-\xi)}{2}}} \Omega(a) d^{\times} a d^{\times} z \\
& =\operatorname{vol}\left(z \in E^{1}: 1+x z \in \varpi_{E}^{\frac{v_{E}(1-\xi)}{2}} \mathcal{O}_{E}\right) \operatorname{vol}\left(F^{\times} \backslash F^{\times} U_{E}\right) \Omega\left(\varpi_{E}^{\frac{v_{E}(1-\xi)}{2}}\right) .
\end{aligned}
$$

The lemma now easily follows if $v(1-\xi) \leq 0$.

It now remains to calculate $\operatorname{vol}\left(\left\{z \in E^{1}: v_{E}(1+x z) \geq 1\right\}\right)$ when $v$ ramifies in $E$ and $v(1-\xi)=v\left(\mathfrak{d}_{E / F}\right)$. In this case we can take $\tau=\sqrt{\varpi}$. Since the volume only depends on $x \bar{x}$ we can assume that $x \in U_{E}^{1}$. Hence we can write $x=x_{1}+x_{2} \sqrt{\varpi}$ with $x_{1} \in U_{F}^{1}$ and $x_{2} \in \mathcal{O}_{F}$. We write $z=\alpha \bar{\alpha}^{-1}$ with $\alpha=\alpha_{1}+\alpha_{2} \sqrt{\varpi}$. Then

$$
\begin{aligned}
1+x z & =\bar{\alpha}^{-1}(\bar{\alpha}+x \alpha) \\
& =\bar{\alpha}^{-1}\left(\alpha_{1}-\alpha_{2} \sqrt{\varpi}+\left(x_{1}+x_{2} \sqrt{\varpi}\right)\left(\alpha_{1}+\alpha_{2} \sqrt{\varpi}\right)\right) \\
& =\bar{\alpha}^{-1}\left(\alpha_{1}\left(1+x_{1}\right)+\alpha_{2} x_{2} \varpi+\left(\alpha_{1} x_{2}+\alpha_{2}\left(x_{1}-1\right)\right) \sqrt{\varpi}\right) .
\end{aligned}
$$

Hence we have $v_{E}(1+x z) \geq 1$ if and only if $v_{E}(\alpha)$ is odd, from which we deduce that

$$
\operatorname{vol}\left(\left\{z \in E^{1}: v_{E}(1+x z) \geq 1\right\}\right)=\frac{1}{2} \operatorname{vol}\left(E^{1}\right) .
$$

Lemma 4.13. Let $v$ be a finite prime which is split in $E$ and such that $n\left(\Omega_{v}\right)=$ 0. Then

$$
I\left(\xi, \mathbf{1}_{v}\right)=\left(\operatorname{vol}\left(U_{F_{v}}\right)\right)^{2} \times \begin{cases}0 & \text { if } v(1-\xi)>0 \\ (v(\xi)+1) & \text { if } v(1-\xi)=0 \\ \Omega_{v}(\xi, 1) \sum_{l=0}^{|v(\xi)|} \Omega_{v}\left(\varpi_{v}^{2 l}, 1\right) & \text { if } v(1-\xi)<0 .\end{cases}
$$

Proof. Let $x=\left(x_{1}, x_{2}\right)$ and $k=v(1-\xi)$. We look at our orbital integral,

$$
I(\xi, \mathbf{1})=\int_{F^{\times} \backslash E^{\times}} \int_{E^{1}} \mathbf{1}\left(\left(\begin{array}{cc}
a & a z x \\
\overline{a z x} & \bar{a}
\end{array}\right)\right) \Omega(a) d^{\times} z d^{\times} a .
$$

A matrix in the integrand of this integral lies in $F^{\times} R^{\times}$if and only if there is a $\lambda \in F^{\times}$such that

1. $v\left(\lambda^{2} a \bar{a}\right)=-v(1-\xi)$

2. $\lambda a \in \mathcal{O}_{E}$

3. $\lambda a(1+z x) \in \mathcal{O}_{E}$. 
It is easy to see from these conditions that if $v(1-\xi) \leq 0$,

$$
I(\xi, \mathbf{1})=\sum_{l=0}^{-k} \int_{F^{\times} \backslash F^{\times}\left(\varpi^{l} U_{F} \times \varpi^{-k-l} U_{F}\right)} \Omega(a) d^{\times} a \sum_{m=-v\left(x_{1}\right)-l}^{-k-l+v\left(x_{2}\right)} \int_{\left(\varpi^{m}, \varpi^{-m}\right) U_{F}} d^{\times} b .
$$

If $k=0$,

$$
I(\xi, \mathbf{1})=(v(\xi)+1) \operatorname{vol}\left(U_{F}\right)^{2} .
$$

If $k<0$, then $v\left(x_{1} x_{2}\right)=k$ and

$$
I(\xi, \mathbf{1})=\sum_{l=0}^{-k} \Omega\left(\varpi^{k+2 l}, 1\right) \operatorname{vol}\left(U_{F}\right)^{2} .
$$

Finally, we compute the regular orbital integral for $v$ archimedean.

Lemma 4.14. For $v \in \Sigma_{\infty}$ and $\xi \in F_{v}$ with $\xi<0$ we have

$$
I\left(\xi, f_{v}\right)=\frac{\operatorname{vol}\left(F_{v}^{\times} \backslash E_{v}^{\times}\right)^{2}}{(1-\xi)^{k_{v}-1}} \sum_{i=0}^{k_{v}-\left|m_{v}\right|-1}\left(\begin{array}{c}
k_{v}+m_{v}-1 \\
i
\end{array}\right)\left(\begin{array}{c}
k_{v}-m_{v}-1 \\
i
\end{array}\right)(-\xi)^{i} .
$$

Proof. By definition of $f_{v}$ we have

$$
f_{v}\left(\left(\begin{array}{cc}
a & 0 \\
0 & \bar{a}
\end{array}\right) \gamma\left(\begin{array}{cc}
b & 0 \\
0 & \bar{b}
\end{array}\right)\right)=\Omega_{v}^{-1}(a b) f_{v}(\gamma) .
$$

Applying Lemma 4.8 gives the result.

\subsubsection{Bounds on $I\left(\xi, \mathbf{1}_{v}\right)$}

We now bound the regular orbital integral in the remaining cases. For a place $v$ of $F$, we fix $x \in E_{v}$ such that $\xi=\epsilon_{v} x \bar{x}$.

Lemma 4.15. Let $v$ be a finite prime. Then

$$
\left|I\left(\xi, \mathbf{1}_{v}\right)\right| \leq \operatorname{vol}\left(F_{v}^{\times} \backslash\left(1+\varpi_{v}^{n\left(\Omega_{v}\right)} \mathcal{O}_{E_{v}}\right)^{\times} F_{v}^{\times}\right) \operatorname{vol}\left(I\left(x, E_{v}^{1}\right)\right) .
$$

Proof. By definition,

$$
I(\xi, \mathbf{1})=\int_{I\left(x, E^{1}\right)} \int_{F^{\times} \backslash E^{\times}} \mathbf{1}\left(a \gamma_{x z}\right) \Omega(a) d^{\times} a d^{\times} z .
$$

For each fixed $z \in I\left(x, E^{1}\right)$ we know that there exists an $a_{x z} \in E^{\times}$such that $a_{x z} \gamma_{x z} \in R^{\times}$. By a change of variables with $a_{x z}$,

$$
|I(\xi, \mathbf{1})| \leq \int_{I\left(x, E^{1}\right)} \int_{F^{\times} \backslash E^{\times}} \mathbf{1}(a) d^{\times} a d^{\times} z .
$$

The lemma now follows by the fact that $R \cap E=\mathcal{O}_{F}+\varpi^{n} \mathcal{O}_{E}$. 
Corollary 4.16. Let $v$ be a finite prime not dividing $\mathfrak{N}$ which is not split in $E$.

Then

$\left|I\left(\xi, \mathbf{1}_{v}\right)\right|$

$\leq \operatorname{vol}\left(F_{v}^{\times} \backslash\left(1+\varpi_{v}^{n\left(\Omega_{v}\right)} \mathcal{O}_{E_{v}}\right)^{\times} F_{v}^{\times}\right) \operatorname{vol}\left(\left\{z \in E_{v}^{1}: v_{E}(1+x z) \geq \frac{v_{E}(1-\xi)}{2}\right\}\right)$.

Proof. If $a \gamma_{x z} \in F^{\times} R^{\times}$then there must be a $\lambda \in F^{\times}$such that $v\left(\lambda^{2} a \bar{a}\right)=$ $-v(1-\xi)$ and $\lambda a(1+z x) \in \mathcal{O}_{F}+\varpi^{n} \mathcal{O}_{E}$. Thus

$$
I\left(x, E^{1}\right) \subseteq\left\{z \in E^{1}: v_{E}(1+x z) \geq \frac{v_{E}(1-\xi)}{2}\right\} .
$$

Lemma 4.17. Let $v$ be a finite prime which is split in $E$.

If $v(1-\xi)<0$, then

$$
\begin{aligned}
& \left|I\left(\xi, \mathbf{1}_{v}\right)\right| \leq(|v(\xi)|+1) \operatorname{vol}\left(F_{v}^{\times} \backslash\left(1+\varpi_{v}^{n\left(\Omega_{v}\right)} \mathcal{O}_{E_{v}}\right)^{\times} F_{v}^{\times}\right) \operatorname{vol}\left(U_{F_{v}}\right) . \\
& \text { If } v(\mathfrak{c}(\Omega)) \geq v(1-\xi) \geq 0, \text { then } \\
& \left|I\left(\xi, \mathbf{1}_{v}\right)\right| \leq \begin{cases}\operatorname{vol}\left(F_{v}^{\times} \backslash\left(1+\varpi_{v}^{n\left(\Omega_{v}\right)} \mathcal{O}_{E_{v}}\right)^{\times} F_{v}^{\times}\right) \operatorname{vol}\left(E^{1} \cap x^{-1}\left(1+\varpi^{\frac{v(1-\xi)}{2}} \mathcal{O}_{E_{v}}\right)\right), & \text { if } v(1-\xi) \text { is even; } \\
0, & \text { if } v(1-\xi) \text { is odd. }\end{cases}
\end{aligned}
$$

Proof. Let $a=\left(a_{1}, a_{2}\right), x=\left(x_{1}, x_{2}\right), z=\left(b, b^{-1}\right)$ and $k=v(1-\xi)$. We take $\tau=(1,0)$. We look at our orbital integral,

$$
I(\xi, \mathbf{1})=\int_{F^{\times} \backslash E^{\times}} \int_{E^{1}} \mathbf{1}\left(\left(\begin{array}{cc}
a & a z x \\
\overline{a z x} & \bar{a}
\end{array}\right)\right) \Omega(a) d^{\times} a d^{\times} z .
$$

It is easy to see that $a \gamma_{x z} \in R^{\times}$if and only if the following conditions are satisfied:

1. $a_{2} \in \frac{1}{\varpi^{n}} \mathcal{O}_{F}$

2. $a_{1} \in\left(-a_{2}+\mathcal{O}_{F}\right) \cap \varpi^{-k-v\left(a_{2}\right)} U_{F}$

3. $a_{2}\left(1+x_{2} b^{-1}\right) \in \mathcal{O}_{F}$

4. $a_{1}\left(1+x_{1} b\right) \in a_{2}\left(1+x_{2} b^{-1}\right)+\varpi^{n} \mathcal{O}_{F}$.

For the intersection in condition (2) to be nonempty, we must have either $v\left(a_{2}\right)=$ $v\left(a_{1}\right)=\frac{-k}{2}$ or $-k \geq v\left(a_{2}\right) \geq 0$ and $v\left(a_{1}\right)=-k-v\left(a_{2}\right)$.

Thus if $k \geq 0$, then we must have $v\left(a_{2}\right)=v\left(a_{1}\right)=\frac{-k}{2}$ and hence,

$$
I\left(x, E^{1}\right) \subseteq E^{1} \cap \frac{1}{x}\left(1+\varpi^{\frac{v(1-\xi)}{2}} \mathcal{O}_{E_{v}}\right) .
$$


If $k<0$, then $-k \geq v\left(a_{2}\right) \geq 0$ and $v\left(a_{1}\right)=-k-v\left(a_{2}\right)$. In this case we must have $v\left(a_{2} x_{2} b^{-1}\right) \geq 0$ and $v\left(a_{1} x_{1} b\right) \geq 0$. Thus $v\left(a_{2} x_{2}\right) \geq v(b) \geq-v\left(a_{1} x_{1}\right)$. Thus

$$
\operatorname{vol}\left(I\left(x, E^{1}\right)\right) \leq \sum_{l=0}^{-k} \sum_{m=k+l-v\left(x_{1}\right)}^{l+v\left(x_{2}\right)} \operatorname{vol}\left(U_{F}\right)=(|v(\xi)|+1) \operatorname{vol}\left(U_{F}\right) .
$$

For the last equality we are using the fact that if $k<0$, then $k=v\left(x_{1} x_{2}\right)$.

We complete the proof by applying Lemma 4.15.

We now bound the volume term that appears in the results above.

Lemma 4.18. Assume that $v$ is unramified in E. Then,

$$
\operatorname{vol}\left(E_{v}^{1} \cap x^{-1}\left(1+\varpi_{v}^{k} \mathcal{O}_{E_{v}}\right)\right) \leq \begin{cases}\operatorname{vol}\left(E_{v}^{1} \cap U_{E_{v}}\right)(1+|v(x \bar{x})|), & \text { if } k=0 \text { and } v \text { split; } \\ \operatorname{vol}\left(E_{v}^{1}\right), & \text { if } k=0 \text { and } v \text { not split; } \\ \operatorname{vol}\left(E_{v}^{1}\right) q_{v}^{-k}\left(1+q_{v}^{-1}\right)^{-1}, & \text { if } k>0 \text { and } v \text { inert; } \\ \operatorname{vol}\left(E_{v}^{1} \cap U_{E_{v}}\right) q_{v}^{-k}\left(1-q_{v}^{-1}\right)^{-1}, & \text { if } k>0 \text { and } v \text { split. }\end{cases}
$$

If $v$ is ramified in $E$ there exists a constant $C\left(E_{v}, F_{v}\right)$ such that,

$$
\operatorname{vol}\left(E_{v}^{1} \cap x^{-1}\left(1+\varpi_{v}^{k} \mathcal{O}_{E_{v}}\right)\right) \leq C\left(E_{v}, F_{v}\right) q_{v}^{-k}
$$

for all $k \geq 0$.

Proof. First we assume that $k=0$. We note that when $E / F$ is not split we have

$$
\operatorname{vol}\left(E^{1} \cap x^{-1} \mathcal{O}_{E}\right)= \begin{cases}\operatorname{vol}\left(E^{1}\right), & \text { if } x \in \mathcal{O}_{E} \\ 0, & \text { otherwise }\end{cases}
$$

Next we assume $E / F$ is split. We write $E=F \oplus F$ and $x=\left(x_{1}, x_{2}\right)$. Then we have

$$
x^{-1} \mathcal{O}_{E}=\left\{\left(\alpha_{1}, \alpha_{2}\right): v\left(\alpha_{1}\right) \geq-v\left(x_{1}\right), v\left(\alpha_{2}\right) \geq-v\left(x_{2}\right)\right\} .
$$

Let $\left(y_{1}, y_{2}\right) \in E^{1}$. Then we have $v\left(y_{1} y_{2}\right)=0$ and hence $y \in \frac{1}{x} \mathcal{O}_{E}$ implies that

$$
-v\left(x_{1}\right) \leq v\left(y_{1}\right) \leq v\left(x_{2}\right) .
$$

Hence the intersection is empty if $v(x \bar{x})>0$, and if $v(x \bar{x}) \leq 0$ then

$$
\operatorname{vol}\left(E^{1} \cap x^{-1} \mathcal{O}_{E}\right)=(1+|v(x \bar{x})|) \operatorname{vol}\left(E^{1} \cap U_{E}\right) .
$$

We now assume $k>0$. If we assume that $E^{1} \cap x^{-1}\left(1+\varpi^{k} \mathcal{O}_{E}\right) \neq \emptyset$ then we clearly have

$$
\operatorname{vol}\left(E^{1} \cap x^{-1}\left(1+\varpi^{k} \mathcal{O}_{E}\right)\right)=\operatorname{vol}\left(E^{1} \cap\left(1+\varpi^{k} \mathcal{O}_{E}\right)\right) .
$$

So we need to compute the order of

$$
\left(E^{1} \cap U_{E}\right) / E^{1} \cap\left(1+\varpi^{k} \mathcal{O}_{E}\right) \stackrel{\sim}{\longrightarrow}\left(E^{1} \cap U_{E}\right)\left(1+\varpi^{k} \mathcal{O}_{E}\right) /\left(1+\varpi^{k} \mathcal{O}_{E}\right) .
$$


Now we have

$$
\left(E^{1} \cap U_{E}\right)\left(1+\varpi^{k} \mathcal{O}_{E}\right)=\left\{x \in U_{E}: N(x) \in N\left(1+\varpi^{k} \mathcal{O}_{E}\right)\right\} .
$$

Hence we are looking to compute the size of the kernel of the map

$$
N: U_{E} /\left(1+\varpi^{k} \mathcal{O}_{E}\right) \rightarrow U_{F} / N\left(1+\varpi^{k} \mathcal{O}_{E}\right) .
$$

When $E / F$ is unramified quadratic this map is surjective and we have

$$
\# U_{E} /\left(1+\varpi^{k} \mathcal{O}_{E}\right)=q^{2 k}\left(1-q^{-2}\right)
$$

and

$$
\# U_{F} / N\left(1+\varpi^{k} \mathcal{O}_{E}\right)=\# U_{F} /\left(1+\varpi^{k} \mathcal{O}_{F}\right)=q^{k}\left(1-q^{-1}\right)
$$

and hence the kernel has order $q^{k}\left(1+q^{-1}\right)$. Next we assume that $E=F \oplus F$, then the norm map is surjective and its kernel has order $q^{k}\left(1-q^{-1}\right)$.

Finally we assume that $E / F$ is ramified. We write the discriminant as $\mathfrak{d}_{E / F}=\mathfrak{p}^{t+1}$. From [Ser62, Corollaire 1, pg 93] we deduce that when $t$ is even and $k>\frac{t}{2}$,

$$
N\left(1+\varpi^{k} \mathcal{O}_{E}\right)=1+\varpi^{k+\frac{t}{2}} \mathcal{O}_{F},
$$

and when $t$ is odd $k \geq \frac{t}{2}$,

$$
N\left(1+\varpi^{k} \mathcal{O}_{E}\right)=1+\varpi^{k+\frac{t+1}{2}} \mathcal{O}_{F} .
$$

Since $\# U_{E} /\left(1+\varpi^{k} \mathcal{O}_{E}\right)=q^{2 k}\left(1-q^{-1}\right)$ we see that when $k>\frac{t}{2}$, the order of the kernel of $N: U_{E} /\left(1+\varpi^{k} \mathcal{O}_{E}\right) \rightarrow U_{F} / N\left(1+\varpi^{k} \mathcal{O}_{E}\right)$ is at least $q^{k-\frac{t+1}{2}}$. Thus there exists some constant $C^{\prime}(E)$ such that for all $k \geq 0$ the order of this kernel is at least $C^{\prime}(E) q^{k}$.

The following lemma is a direct consequence of Corollary 4.16 and Lemmas 4.17 and 4.18 , and the fact that

$$
\operatorname{vol}\left(F_{v}^{\times} \backslash F_{v}^{\times}\left(1+\varpi_{v}^{n\left(\Omega_{v}\right)} \mathcal{O}_{E_{v}}\right)^{\times}\right)=\operatorname{vol}\left(U_{F_{v}} \backslash U_{E_{v}}\right) q_{v}^{-n\left(\Omega_{v}\right)} L\left(1, \eta_{v}\right) .
$$

Lemma 4.19. Assume $n\left(\Omega_{v}\right)>0$, and let $k=\frac{v_{F}(1-\xi)}{2}$. There exists a constant $C\left(E_{v}, F_{v}\right)$ that is equal to one for all $v$ unramified in $E$ and such that

$$
\begin{aligned}
\left|I\left(\xi, \mathbf{1}_{v}\right)\right| \leq & q_{v}^{-n\left(\Omega_{v}\right)} L\left(1, \eta_{v}\right) \operatorname{vol}\left(U_{F_{v}} \backslash U_{E_{v}}\right) \operatorname{vol}\left(E_{v}^{1} \cap U_{E_{v}}\right) C\left(E_{v}, F_{v}\right) \\
& \times \begin{cases}q_{v}^{-k} L\left(1, \eta_{v}\right) & \text { when } k>0 \\
1 & \text { when } k \leq 0 \text { and } v \text { is not split } \\
1+\left|v_{F}(\xi)\right| & \text { when } k \leq 0 \text { and } v \text { is split. }\end{cases}
\end{aligned}
$$




\subsection{Global calculations}

We recall that

$$
I(f)=\sum_{\xi \in \epsilon N E^{\times}} I(\xi, f)+\operatorname{vol}\left(\mathbf{A}_{F}^{\times} E^{\times} \backslash \mathbf{A}_{E}^{\times}\right)\left[I(0, f)+\delta\left(\Omega^{2}\right) I(\infty, f)\right] .
$$

We now apply the local calculations of the previous sections to this global distribution. We denote by

$$
I_{r e g}(f)=\sum_{\xi \in \epsilon N E^{\times}} I(\xi, f)
$$

and

$$
I_{\text {irreg }}(f)=\operatorname{vol}\left(\mathbf{A}_{F}^{\times} E^{\times} \backslash \mathbf{A}_{E}^{\times}\right)\left[I(0, f)+\delta\left(\Omega^{2}\right) I(\infty, f)\right] .
$$

Thus

$$
I(f)=I_{\text {reg }}(f)+I_{\text {irreg }}(f) .
$$

We begin by computing $I_{\text {irreg }}(f)$.

Proposition 4.20. For $f$ as defined in Section 3.1,

$I_{\text {irreg }}(f)=\frac{2^{[F: \mathbf{Q}]+1} L(1, \eta) L_{S(\Omega)}(1, \eta) \sqrt{\left|\Delta_{F}\right|}}{\sqrt{c(\Omega)} \sqrt{\left|\Delta_{E}\right|}}\left(1+\delta\left(\Omega^{2}\right) \delta(\mathfrak{N}) \prod_{v \in \Sigma_{\infty}}(-1)^{k_{v}-1}\right) \tilde{I}\left(f_{\mathfrak{p}}\right)$,

where $\delta(\mathfrak{N})=0$ if $\mathfrak{N} \neq \mathcal{O}_{F}$ and $\delta\left(\mathcal{O}_{F}\right)=1$.

Proof. By Lemmas 4.1, 4.2, 4.4, 4.5, 4.6 and 4.7, and Corollary 4.9,

$$
\operatorname{vol}\left(\mathbf{A}_{F}^{\times} E^{\times} \backslash \mathbf{A}_{E}^{\times}\right)\left[I(0, f)+\delta\left(\Omega^{2}\right) I(\infty, f)\right]
$$

is equal to

$2 L(1, \eta) \tilde{I}\left(f_{\mathfrak{p}}\right) \prod_{v<\infty} \operatorname{vol}\left(F_{v}^{\times} \backslash F_{v}^{\times}\left(1+\varpi_{v}^{n\left(\Omega_{v}\right)} \mathcal{O}_{E_{v}}\right)^{\times}\right) \prod_{v \in \Sigma_{\infty}} \operatorname{vol}\left(F_{v}^{\times} \backslash E_{v}^{\times}\right)\left(1+\delta\left(\Omega^{2}\right) \delta(\mathfrak{N})(-1)^{k_{v}-1}\right)$.

Recall that for $v \in \Sigma_{\infty}$,

$$
\operatorname{vol}\left(F_{v}^{\times} \backslash E_{v}^{\times}\right)=\operatorname{vol}\left(\mathbf{R}^{\times} \backslash \mathbf{C}^{\times}\right)=2,
$$

and for $v$ finite we have

$\operatorname{vol}\left(F_{v}^{\times} \backslash F_{v}^{\times}\left(1+\varpi_{v}^{n\left(\Omega_{v}\right)} \mathcal{O}_{E_{v}}\right)^{\times}\right)= \begin{cases}\operatorname{vol}\left(U_{F_{v}} \backslash U_{E_{v}}\right), & \text { if } v \notin S(\Omega) ; \\ \operatorname{vol}\left(U_{F_{v}} \backslash U_{E_{v}}\right) q_{v}^{-n\left(\Omega_{v}\right)} L\left(1, \eta_{v}\right), & \text { if } v \in S(\Omega) .\end{cases}$

The proposition now follows.

We now consider the regular terms in the trace formula. For each $v \in \Sigma_{\infty}$ we let $\iota_{v}$ denote the corresponding embedding $F \hookrightarrow \mathbf{R}$. We note that $\xi \in \epsilon N E^{\times}$ if and only if 
1. $\iota_{v}(\xi)<0$ for all $v \in \Sigma_{\infty}$,

2. $v(\xi)$ is odd for all $v \mid \mathfrak{N}$, and

3. $\eta_{v}(\xi)=1$ for all finite $v \nmid \mathfrak{N}$.

We define

$$
X_{\mathfrak{p}}=\left\{\gamma \in R_{\mathfrak{p}}: \varpi_{\mathfrak{p}}^{-1} \gamma \notin R\right\} .
$$

For $f_{\mathfrak{p}} \in \mathcal{H}\left(G\left(f_{\mathfrak{p}}\right), R_{\mathfrak{p}}^{\times}\right)$we define

$$
n\left(f_{\mathfrak{p}}\right)=\max \left\{v_{\mathfrak{p}}(\operatorname{det} \gamma): \gamma \in X_{\mathfrak{p}}, f_{\mathfrak{p}}(\gamma) \neq 0\right\}
$$

and set $\mathfrak{I}\left(f_{\mathfrak{p}}\right)=\mathfrak{p}^{n\left(f_{\mathfrak{p}}\right)}$.

We denote by $S\left(\Omega, \mathfrak{N}, f_{\mathfrak{p}}\right)$ the set of $\xi \in \epsilon N E^{\times}$such that

1. $v(\xi) \geq 1$ for all $v \mid \mathfrak{N}$, and

2. $(1-\xi)^{-1} \in\left(\mathfrak{c}(\Omega) \mathfrak{d}_{E / F} \mathfrak{I}\left(f_{\mathfrak{p}}\right)\right)^{-1}$.

Lemma 4.21. We have

$$
I_{\text {reg }}(f)=\sum_{\xi \in S\left(\Omega, \mathfrak{N}, f_{\mathfrak{p}}\right)} I(\xi, f) .
$$

Furthermore, the set $S\left(\Omega, \mathfrak{N}, f_{\mathfrak{p}}\right)$ is finite, and empty when $|\mathfrak{N}| \geq d_{E / F}\left(c(\Omega)\left|\mathfrak{I}\left(f_{\mathfrak{p}}\right)\right|\right)^{h_{F}}$.

Proof. The fact that $I_{\text {reg }}(f)$ is supported on $S\left(\Omega, \mathfrak{N}, f_{\mathfrak{p}}\right)$ follows from Lemmas 4.10 and 4.11. Suppose now that $\xi \in S\left(\Omega, \mathfrak{N}, f_{\mathfrak{p}}\right)$. We fix $0 \neq x \in$ $\mathfrak{c}(\Omega) \mathfrak{d}_{E / F} \mathfrak{I}\left(f_{\mathfrak{p}}\right)$, then

$$
(1-\xi)^{-1} x=m \in \mathcal{O}_{F}
$$

and hence

$$
\xi=\frac{m-x}{m} .
$$

We now take $v \in \Sigma_{\infty}$ and consider $\iota_{v}: F \hookrightarrow \mathbf{R}$, then since $\iota_{v}(\xi)<0$ it follows that

$$
\left|\iota_{v}(m-x)\right|<\left|\iota_{v}(x)\right| .
$$

The finiteness of $S\left(\Omega, \mathfrak{N}, f_{\mathfrak{p}}\right)$ now follows from the finiteness of

$$
\left\{y \in \mathcal{O}_{F}:\left|\iota_{v}(y)\right|<\left|\iota_{v}(x)\right| \text { for all } v \in \Sigma_{\infty} \text { and } x \in \mathfrak{c}(\Omega) \mathfrak{d}_{E / F} \mathfrak{I}\left(f_{\mathfrak{p}}\right)\right\} .
$$

We note that since $v(\xi) \geq 1$ for all $v \mid \mathfrak{N}$ we also require $m-x \in \mathfrak{N}$. Hence $S\left(\Omega, \mathfrak{N}, f_{\mathfrak{p}}\right)$ is empty whenever

$\left\{y \in \mathcal{O}_{F}:\left|\iota_{v}(y)\right|<\left|\iota_{v}(x)\right|\right.$ for all $v \in \Sigma_{\infty}$ and $\left.x \in \mathfrak{c}(\Omega) \mathfrak{d}_{E / F} \mathfrak{I}\left(f_{\mathfrak{p}}\right)\right\} \cap \mathfrak{N}=\{0\}$,

which is clearly the case when $|\mathfrak{N}| \geq d_{E / F}\left|\mathfrak{c}(\Omega) \mathfrak{I}\left(f_{\mathfrak{p}}\right)\right|^{h_{F}}$. 
Corollary 4.22. For $\mathfrak{N}$ sufficiently large, e.g. for $\mathfrak{N}$ with absolute norm at least $d_{E / F}\left(c(\Omega)\left|\mathfrak{I}\left(f_{\mathfrak{p}}\right)\right|\right)^{h_{F}}$, we have

$I(f)=\frac{2^{[F: \mathbf{Q}]+1} L(1, \eta) L_{S(\Omega)}(1, \eta) \sqrt{\left|\Delta_{F}\right|}}{\sqrt{c(\Omega)} \sqrt{\left|\Delta_{E}\right|}}\left(1+\delta\left(\Omega^{2}\right) \delta(\mathfrak{N}) \prod_{v \in \Sigma_{\infty}}(-1)^{k_{v}-1}\right) \tilde{I}\left(f_{\mathfrak{p}}\right)$.

We note that under certain simplifying assumptions we can explicitly compute the regular terms in the geometric side of the trace formula.

We define, for integers $k$ and $m$ with $|m|<k$, polynomials

$$
P_{k, m}(x)=\frac{1}{(1-x)^{k-1}} \sum_{i=0}^{k-|m|-1}\left(\begin{array}{c}
k+m-1 \\
i
\end{array}\right)\left(\begin{array}{c}
k-m-1 \\
i
\end{array}\right)(-x)^{i} .
$$

For an ideal $\mathfrak{a} \subset \mathcal{O}_{F}$ we define

$$
R_{E}(\mathfrak{a})=\left\{\mathfrak{b} \subset \mathcal{O}_{E}: N_{E / F}(\mathfrak{b})=\mathfrak{a}\right\} .
$$

For non-zero ideals $\mathfrak{a}, \mathfrak{b} \in \mathcal{O}_{F}$ we define

$$
\sigma(\mathfrak{a}, \mathfrak{b})=\#\left\{\mathfrak{c} \subset \mathcal{O}_{F}: \mathfrak{c} \mid \mathfrak{a}+\mathfrak{b}\right\} .
$$

When $\Omega$ is unramified we regard its finite part as a character on the group of fractional ideals of $F$.

Proposition 4.23. Assume that $\Omega$ is unramified, $f_{\mathfrak{p}}=\mathbf{1}_{\mathfrak{p}}$ and $E / F$ is unramified at the even places of $F$. Let $d \in \mathcal{O}_{F}$ be a generator of $\mathfrak{d}_{E / F}$. Then we have $I(f)$ equal to the sum of

$$
\frac{2^{[F: \mathbf{Q}]+1} L(1, \eta) \sqrt{\left|\Delta_{F}\right|}}{\sqrt{c(\Omega)} \sqrt{\left|\Delta_{E}\right|}}\left(1+\delta\left(\Omega^{2}\right) \delta(\mathfrak{N}) \prod_{v \in \Sigma_{\infty}}(-1)^{k_{v}-1}\right)
$$

and

$4^{[F: \mathbf{Q}]} \frac{\left|\Delta_{F}\right|}{\left|\Delta_{E}\right|} \sum_{n}\left|R_{E}\left(n \mathfrak{N}^{-1}\right)\right| \sigma\left(\mathfrak{d}_{E / F},(n+d)\right) \sum_{\mathfrak{a} \in R_{E}((n+d))} \Omega\left(\mathfrak{D}_{E / F}^{-1} \mathfrak{a}\right) \prod_{v \in \Sigma_{\infty}} P_{k_{v}, m_{v}}\left(\iota_{v}\left(\frac{n}{n+d}\right)\right)$,

with the outer sum taken over the finite set of $n \in \mathfrak{N}$ such that

1. $\eta_{v}\left(1+\frac{d}{n}\right)=1$ for all $v \mid \mathfrak{d}_{E / F}$, and

2. $\iota_{v}(n)$ lies between $-\iota_{v}(d)$ and 0 for all $v \in \Sigma_{\infty}$.

Proof. Let $\xi \in \epsilon N E^{\times}$and let $v$ be a finite place of $F$. When $v$ splits in $E$ we have, with the notation of Lemma 4.13,

- $v(1-\xi)>0 \Longrightarrow I\left(\xi, f_{v}\right)=0$, 
- $v(1-\xi)=0 \Longrightarrow I\left(\xi, f_{v}\right)=(1+v(\xi)) \operatorname{vol}\left(U_{F_{v}}\right)^{2}$,

- $v(1-\xi)<0 \Longrightarrow I\left(\xi, f_{v}\right)=\sum_{i=0}^{|v(\xi)|} \Omega\left(\varpi_{v}^{i}, \varpi_{v}^{|v(\xi)|-i}\right) \operatorname{vol}\left(U_{F_{v}}\right)^{2}$.

When $v \nmid \mathfrak{N}$ is inert and unramified in $E$ we have $\Omega_{v}$ trivial and, by Lemma 4.12 ,

- $v(1-\xi)>0 \Longrightarrow I\left(\xi, f_{v}\right)=0$,

- $v(1-\xi)$ odd and $\leq 0 \Longrightarrow I\left(\xi, f_{v}\right)=0$,

- $v(1-\xi)$ even and $\leq 0 \Longrightarrow I\left(\xi, f_{v}\right)=\operatorname{vol}\left(U_{F_{v}} \backslash U_{E_{v}}\right)^{2}$.

When $v \mid \mathfrak{N}$ we have $\Omega_{v}$ trivial and, by Lemma 4.10,

- $v(\xi) \leq 0 \Longrightarrow I\left(\xi, f_{v}\right)=0$,

- $v(\xi) \geq 1 \Longrightarrow I\left(\xi, f_{v}\right)=\operatorname{vol}\left(U_{F_{v}} \backslash U_{E_{v}}\right)^{2}$.

When $v$ is odd and ramified in $E$ we have $\Omega_{v}^{2}$ trivial and, by Lemma 4.12,

- $v(1-\xi)>1 \Longrightarrow I\left(\xi, f_{v}\right)=0$,

- $v(1-\xi)=1 \Longrightarrow I\left(\xi, f_{v}\right)=\operatorname{vol}\left(U_{F_{v}} \backslash U_{E_{v}}\right)^{2} \Omega\left(\varpi_{E_{v}}\right)^{-1}$,

- $v(1-\xi) \leq 0 \Longrightarrow I\left(\xi, f_{v}\right)=2 \Omega\left(\varpi_{E_{v}}\right)^{\frac{v_{E}(1-\xi)}{2}} \operatorname{vol}\left(U_{F_{v}} \backslash U_{E_{v}}\right)^{2}$.

And at $v \in \Sigma_{\infty}$ we have, by Lemma 4.14 ,

$$
I\left(\xi, f_{v}\right)=\operatorname{vol}\left(F_{v}^{\times} \backslash E_{v}^{\times}\right)^{2} P_{m_{v}, k_{v}}\left(\iota_{v}(\xi)\right) .
$$

As in the proof of Lemma 4.21 we note that if $I(\xi, f) \neq 0$ then we have

$$
\xi=\frac{n}{n+d}
$$

with $n \in \mathfrak{N}$. Furthermore such an element lies in $\epsilon N E^{\times}$if and only if

- $R_{E}\left(n \mathfrak{N}^{-1}\right)$ and $R_{E}((n+d))$ are non-empty,

- $\eta_{v}\left(1+\frac{d}{n}\right)=1$ for all $v \mid \mathfrak{d}_{E / F}$, and

- $\iota_{v}(n)$ lies between $\iota_{v}(0)$ and $-\iota_{v}(d)$ for all $v \in \Sigma_{\infty}$.

Suppose now we fix such an $n$. We have

$$
I(\xi, f)=\prod_{v} I\left(\xi, f_{v}\right)
$$

and we now determine the contribution to this product from each place $v$ depending on its behavior in the extension $E$.

For a finite place $v$ of $F$ and an ideal $\mathfrak{a} \subset \mathcal{O}_{F}$ we define

$$
R_{E_{v}}(\mathfrak{a})=\left\{\mathfrak{b} \subset \mathcal{O}_{E_{v}}: N_{E_{v} / F_{v}} \mathfrak{b}=\mathfrak{a} \mathcal{O}_{F_{v}}\right\} .
$$


The contribution from the places $v \mid \mathfrak{N}$ is,

$$
\prod_{v \mid \mathfrak{N}} \operatorname{vol}\left(U_{F_{v}} \backslash U_{E_{v}}\right)^{2}\left|R_{E_{v}}\left(n \mathfrak{N}^{-1}\right)\right|,
$$

and furthermore for such $v$ we have $R_{E_{v}}((n+d))=\left\{\mathcal{O}_{E_{v}}\right\}$. For finite $v \nmid \mathfrak{N}$ which are inert in $E$ we get

$$
\prod_{v \nmid \mathfrak{N}, \text { inert }} \operatorname{vol}\left(U_{F_{v}} \backslash U_{E_{v}}\right)^{2}\left|R_{E_{v}}((n))\right|\left|R_{E_{v}}((n+d))\right| .
$$

For finite $v$ which split in $E$ we get

$$
\prod_{v<\infty, \text { split }} \operatorname{vol}\left(U_{F_{v}} \backslash U_{E_{v}}\right)^{2}\left|R_{E_{v}}((n))\right| \sum_{\mathfrak{a} \in R_{E_{v}}((n+d))} \Omega_{v}(\mathfrak{a}),
$$

since for such $v$ when $v(1-\xi)=0$ we have $v(\xi)=v(n)$ and $v(n+d)=0$, and when $v(1-\xi)<0$ we have $v(n)=0$ and $|v(\xi)|=v(n+d)$. For $v$ ramified in $E$ the contribution to $I(\xi, f)$ is

$$
\sigma\left(\mathfrak{d}_{E / F},(n+d)\right) \prod_{v \mid \mathfrak{d}_{E / F}} \operatorname{vol}\left(U_{F_{v}} \backslash U_{E_{v}}\right)^{2}\left|R_{E_{v}}((n))\right| \sum_{\mathfrak{a} \in R_{E_{v}}((n+d))} \Omega_{v}\left(\mathfrak{D}_{E / F}^{-1} \mathfrak{a}\right),
$$

since for such $v, \Omega_{v}^{2}$ is trivial and $v(1-\xi)=v\left(\mathfrak{d}_{E / F}\right)-v(n+d)$. Finally for $v \in \Sigma_{\infty}$ we have $I\left(\xi, f_{v}\right)=4 P_{k_{v}, m_{v}}\left(\iota_{v}(n /(n+d))\right)$.

Putting these local calculations together gives the sum in the statement of the Proposition and combining it with the calculation of the irregular terms from Proposition 4.20 gives the result.

\section{A measure on the Hecke algebra}

The goal of this section is to find the measure $\mu$ such that for $f_{\mathfrak{p}} \in \mathcal{H}\left(G_{\mathfrak{p}}, Z_{\mathfrak{p}} R_{\mathfrak{p}}^{\times}\right)$,

$$
\tilde{I}\left(f_{\mathfrak{p}}\right)=\int_{-2}^{2} \hat{f}_{\mathfrak{p}}(x) \mu
$$

where $\tilde{I}\left(f_{\mathfrak{p}}\right)$ is defined in Section 4.1. We begin by recalling standard facts about distributions on Hecke algebras which come from the Plancherel formula; alternatively see [Ser97, Section 2].

\subsection{An application of the Plancherel formula}

We assume throughout this subsection that $F$ is a non-archimedean local field of characteristic zero. We let $q$ denote the order of the residue field of $F$. We let $G^{\prime}=\operatorname{PGL}(2, F)$ and $K=\operatorname{PGL}\left(2, \mathcal{O}_{F}\right)$. We fix a Haar measure on $G^{\prime}$ which gives $K$ volume one. For $n \geq 0$ we denote by $f_{n}$ the characteristic function of

$$
K\left(\begin{array}{cc}
\varpi^{n} & 0 \\
0 & 1
\end{array}\right) K
$$


For $s \in i \mathbf{R}$ let $\pi_{s}$ denote the unramified principal series representation of $G^{\prime}$ unitarily induced from

$$
\left(\begin{array}{ll}
a & 0 \\
0 & b
\end{array}\right) \mapsto\left|\frac{a}{b}\right|^{s}
$$

For $f \in \mathcal{H}\left(G^{\prime}, K\right)$ and $x \in[-2,+2]$ we define

$$
\hat{f}(x)=\operatorname{Tr} \pi_{s}(f),
$$

where $x=q^{s}+q^{-s}$. We have (see [RR05, Lemma 9]) $\hat{f}_{0} \equiv 1$ and for $n>0$,

$$
\hat{f}_{n}(x)=q^{\frac{n}{2}}\left(q^{n s}+q^{-n s}+\left(1-q^{-1}\right)\left(q^{(n-2) s}+q^{(n-4) s}+\ldots+q^{-(n-2) s}\right)\right) .
$$

On the interval $[-2,+2]$ we take the Sato-Tate measure

$$
\mu_{\infty}=\frac{\sqrt{4-x^{2}}}{2 \pi} d x
$$

and the spherical Plancherel measure on $\operatorname{PGL}(2, F)$,

$$
\mu_{q}=\frac{q+1}{\left(q^{\frac{1}{2}}+q^{-\frac{1}{2}}\right)^{2}-x^{2}} \mu_{\infty}
$$

By the Plancherel formula we have,

Lemma 5.1. For all $f \in \mathcal{H}\left(G^{\prime}, K\right)$ we have

$$
f\left(\begin{array}{ll}
1 & 0 \\
0 & 1
\end{array}\right)=\int_{-2}^{2} \hat{f}(x) \mu_{q}
$$

and

$$
f\left(\begin{array}{cc}
\varpi^{m} & 0 \\
0 & 1
\end{array}\right)=\frac{1}{\left(1+q^{-1}\right) q^{m}} \int_{-2}^{2} \hat{f}(x) \hat{f}_{m}(x) \mu_{q},
$$

for $m>0$.

In particular we note the following corollary.

Corollary 5.2. We have

$$
f\left(\begin{array}{ll}
1 & 0 \\
0 & 1
\end{array}\right)+\delta f\left(\begin{array}{cc}
\varpi & 0 \\
0 & 1
\end{array}\right)=\frac{1}{q+1} \int_{-2}^{2} \hat{f}(x)\left(1+q^{\frac{1}{2}} \delta x+q\right) \mu_{q},
$$

and

$$
f\left(\begin{array}{ll}
1 & 0 \\
0 & 1
\end{array}\right)-f\left(\begin{array}{cc}
\varpi^{2} & 0 \\
0 & 1
\end{array}\right)=\int_{-2}^{2} \hat{f}(x) \mu_{\infty}
$$

We define for $\delta \in \mathbf{C}$ a distribution

$$
\Lambda_{\delta}: f \mapsto \sum_{n \in \mathbf{Z}} \delta^{n} f\left(\begin{array}{cc}
\varpi^{n} & 0 \\
0 & 1
\end{array}\right)
$$

on $\mathcal{H}\left(G^{\prime}, K\right)$. Then we have the following. 
Lemma 5.3. For $f \in \mathcal{H}\left(G^{\prime}, K\right)$ and $\delta$ with $|\delta|=1$,

$$
\Lambda_{\delta}(f)=\int_{-2}^{2} \hat{f}(x) \frac{\left(1-q^{-1}\right)}{\left(1-\delta q^{-\frac{1}{2}} x+\delta^{2} q^{-1}\right)\left(1-\delta^{-1} q^{-\frac{1}{2}} x+\delta^{-2} q^{-1}\right)} \mu_{\infty} .
$$

Proof. This follows by a short calculation using Lemma 5.1, Formula 4 and that,

$$
f\left(\begin{array}{cc}
\varpi^{m} & \\
& 1
\end{array}\right)=f\left(\begin{array}{cc}
\varpi^{-m} & \\
& 1
\end{array}\right) .
$$

Alternatively, one can argue as in [RR05, Section 6] in the case $\delta=1$.

\subsection{The distribution $\tilde{I}$}

For $\alpha, \beta \in \mathbf{C}$ we let $\rho(\alpha, \beta)$ denote the unramified representation of $\operatorname{GL}\left(2, F_{\mathfrak{p}}\right)$ with Satake parameters $\{\alpha, \beta\}$. For $x \in[-2,+2]$ we define $\alpha_{x} \in \mathbf{C}$ to be such that $\alpha_{x}+\alpha_{x}^{-1}=x$; of course $\alpha_{x}$ is not well defined, however, all constructions below will depend only on $x$, and not on the choice of $\alpha_{x}$. We then define

$$
\mu_{\mathfrak{p}, E, \Omega}=L\left(1 / 2, \rho\left(\alpha_{x}, \alpha_{x}^{-1}\right)_{E_{\mathfrak{p}}} \otimes \Omega_{\mathfrak{p}}\right) \frac{\sqrt{4-x^{2}}}{2 \pi} d x,
$$

where $\rho\left(\alpha_{x}, \alpha_{x}^{-1}\right)_{E_{\mathfrak{p}}}$ denotes the base change of $\rho\left(\alpha_{x}, \alpha_{x}^{-1}\right)$ to $\mathrm{GL}\left(2, E_{\mathfrak{p}}\right)$. We note that,

$L\left(1 / 2, \rho\left(\alpha_{x}, \alpha_{x}^{-1}\right)_{E_{\mathfrak{p}}} \otimes \Omega_{\mathfrak{p}}\right)=1$, when $\Omega_{\mathfrak{p}}$ is ramified and,

$L\left(1 / 2, \rho\left(\alpha_{x}, \alpha_{x}^{-1}\right)_{E_{\mathfrak{p}}} \otimes \Omega_{\mathfrak{p}}\right)=$

$$
\begin{cases}\left(1-x \Omega\left(\varpi_{E_{\mathfrak{p}}}\right) q_{\mathfrak{p}}^{-\frac{1}{2}}+\Omega\left(\varpi_{E_{\mathfrak{p}}}\right)^{2} q_{\mathfrak{p}}^{-1}\right)^{-1}\left(1-\frac{x}{\Omega\left(\varpi_{E_{\mathfrak{p}}}\right)} q_{\mathfrak{p}}^{-\frac{1}{2}}+\frac{1}{\Omega\left(\varpi_{E_{\mathfrak{p}}}\right)^{2}} q_{\mathfrak{p}}^{-1}\right)^{-1}, & \text { for } \mathfrak{p} \text { split, } \\ \left(\left(1+q_{\mathfrak{p}}^{-1}\right)^{2}-x^{2} q_{\mathfrak{p}}^{-1}\right)^{-1}, & \text { for } \mathfrak{p} \text { inert, } \\ \left(1-x \Omega\left(\varpi_{E_{\mathfrak{p}}}\right) q_{\mathfrak{p}}^{-\frac{1}{2}}+q_{\mathfrak{p}}^{-1}\right)^{-1}, & \text { for } \mathfrak{p} \text { ramified }\end{cases}
$$

when $\Omega_{\mathfrak{p}}$ is unramified, where $\varpi_{E_{\mathfrak{p}}}$ is defined in Section 4 . We note that

$$
\int_{-2}^{2} \mu_{\mathfrak{p}, E, \Omega}= \begin{cases}L\left(1, \eta_{\mathfrak{p}}\right), & \text { if } \Omega \text { is unramified at } \mathfrak{p} \\ 1, & \text { otherwise. }\end{cases}
$$

We note for future reference the relationship between $\mu_{\mathfrak{p}, E, \Omega}$ and the spherical Plancherel measure $\mu_{\mathfrak{p}}$ on $\operatorname{PGL}\left(2, F_{\mathfrak{p}}\right)$ is given by

$$
\mu_{\mathfrak{p}, E, \Omega}=\frac{L\left(1 / 2, \rho\left(\alpha_{x}, \alpha_{x}^{-1}\right)_{E_{\mathfrak{p}}} \otimes \Omega_{\mathfrak{p}}\right)}{L\left(1, \rho\left(\alpha_{x}, \alpha_{x}^{-1}\right), A d\right)} L\left(2,1_{F_{\mathfrak{p}}}\right) \mu_{\mathfrak{p}} .
$$

We recall $\tilde{I}\left(f_{\mathfrak{p}}\right)$ is defined in Section 4.1 . 
Lemma 5.4. For all $f_{\mathfrak{p}} \in \mathcal{H}\left(G\left(F_{\mathfrak{p}}\right), Z_{\mathfrak{p}} R_{\mathfrak{p}}^{\times}\right)$we have

$$
\tilde{I}\left(f_{\mathfrak{p}}\right)=\frac{\int_{-2}^{2} \hat{f}_{\mathfrak{p}}(x) \mu_{\mathfrak{p}, E, \Omega}}{L\left(1, \eta_{\mathfrak{p}}\right)},
$$

if $\Omega$ is unramified at $\mathfrak{p}$ and we have

$$
\tilde{I}\left(f_{\mathfrak{p}}\right)=\int_{-2}^{2} \hat{f}_{\mathfrak{p}}(x) \mu_{\mathfrak{p}, E, \Omega}
$$

if $\Omega$ is ramified at $\mathfrak{p}$.

Proof. We fix an isomorphism $D_{\mathfrak{p}} \stackrel{\sim}{\longrightarrow} M\left(2, F_{\mathfrak{p}}\right)$ and an embedding $E_{\mathfrak{p}} \hookrightarrow M\left(2, F_{\mathfrak{p}}\right)$ such that $M\left(2, \mathcal{O}_{F_{\mathfrak{p}}}\right) \cap E=\mathcal{O}_{F}+\varpi_{\mathfrak{p}}^{n\left(\Omega_{\mathfrak{p}}\right)} \mathcal{O}_{E_{\mathfrak{p}}}$ as in the proof of Lemma 4.3. We now prove the lemma on a case by case basis.

First we assume that $n\left(\Omega_{\mathfrak{p}}\right)>0$. Then we have, by Lemmas 4.4, 4.5, 4.6 and Corollary 5.2,

$$
\tilde{I}\left(f_{\mathfrak{p}}\right)=f_{\mathfrak{p}}\left(\begin{array}{ll}
1 & 0 \\
0 & 1
\end{array}\right)-f_{\mathfrak{p}}\left(\begin{array}{cc}
\varpi_{\mathfrak{p}}^{2} & 0 \\
0 & 1
\end{array}\right)=\int_{-2}^{2} \hat{f}_{\mathfrak{p}}(x) \mu_{\infty}
$$

On the other hand in this case we clearly have $\mu_{\mathfrak{p}, E, \Omega}=\mu_{\infty}$.

Next we assume that $\mathfrak{p}$ is unramified and inert in $E$ and $\Omega$ is unramified at $\mathfrak{p}$. Then from Lemmas 4.4 and 5.1 we have

$$
\tilde{I}\left(f_{\mathfrak{p}}\right)=\int_{-2}^{2} \hat{f}_{\mathfrak{p}}(x) \mu_{q_{\mathfrak{p}}}=\int_{-2}^{2} \hat{f}_{\mathfrak{p}}(x) \mu_{\mathfrak{p}, E, \Omega}
$$

Next we assume that $\mathfrak{p}$ splits in $E$ and $\Omega$ is unramified above $\mathfrak{p}$. We write $\Omega_{\mathfrak{p}}=\left(\chi, \chi^{-1}\right)$. Then from Lemmas 4.6 and 5.3 we have

$$
\tilde{I}\left(f_{\mathfrak{p}}\right)=\sum_{m \in \mathbf{Z}} \chi\left(\varpi_{\mathfrak{p}}^{m}\right) f_{\mathfrak{p}}\left(\begin{array}{cc}
\varpi_{\mathfrak{p}}^{m} & 0 \\
0 & 1
\end{array}\right)=\int_{-2}^{2} \hat{f}_{\mathfrak{p}}(x) \mu_{\mathfrak{p}, E, \Omega} .
$$

Finally we assume that $\mathfrak{p}$ is ramified in $E$ and $\Omega$ is unramified at $\mathfrak{p}$. Then from Lemma 4.5 and Corollary 5.2 we have

$$
\tilde{I}\left(f_{\mathfrak{p}}\right)=f\left(\begin{array}{ll}
1 & 0 \\
0 & 1
\end{array}\right)+\Omega(\tau) f\left(\begin{array}{cc}
\varpi_{\mathfrak{p}} & 0 \\
0 & 1
\end{array}\right)=\int_{-2}^{2} \hat{f}_{\mathfrak{p}}(x) \mu_{\mathfrak{p}, E, \Omega}
$$

\section{Main results}

We now combine the calculations of Sections 3 and 4 to obtain the main results of this paper. 


\subsection{Average $L$-values}

By Propositions 3.9, 4.20 and Lemma 4.21 we see that we have an exact formula for

$$
\sum_{\pi \in \mathcal{F}(\mathfrak{N}, 2 k)} \frac{L\left(1 / 2, \pi_{E} \otimes \Omega\right)}{L(1, \pi, A d)} \hat{f}_{\mathfrak{p}}\left(\pi_{\mathfrak{p}}\right),
$$

in terms of orbital integrals. We now write down the formula precisely under certain further assumptions for which we have computed all the necessary orbital integrals.

Combining Proposition 3.9, Corollary 4.22 and Lemma 5.4 we get the following.

Theorem 6.1. Let $E$ be a $C M$ extension of a totally real number field $F$. Let $\mathfrak{N} \subset \mathcal{O}_{F}$ be an ideal such that each prime dividing $\mathfrak{N}$ is unramified and inert in $E$ and that the number of primes dividing $\mathfrak{N}$ has the same parity as $[F: \mathbf{Q}]$. Let $\Omega: \mathbf{A}_{F}^{\times} E^{\times} \backslash \mathbf{A}_{E}^{\times} \rightarrow \mathbf{C}^{\times}$be a character which is unramified outside of $\mathfrak{N}$ and such that for $v \in \Sigma_{\infty}$ the weight $m_{v}$ of $\Omega_{v}$ is strictly less than $k_{v}$. Let $f_{\mathfrak{p}} \in \mathcal{H}\left(G\left(F_{\mathfrak{p}}\right), Z_{\mathfrak{p}} R_{\mathfrak{p}}^{\times}\right)$. Then for $|\mathfrak{N}| \geq d_{E / F}\left(c(\Omega)\left|\mathfrak{I}\left(f_{\mathfrak{p}}\right)\right|\right)^{h_{F}}$,

$$
\frac{2^{[F: \mathbf{Q}]}}{|\mathfrak{N}|}\left(\begin{array}{c}
2 \boldsymbol{k}-2 \\
\boldsymbol{k}+\boldsymbol{m}-1
\end{array}\right) \sum_{\pi \in \mathcal{F}(\mathfrak{N}, 2 \boldsymbol{k})} \frac{L\left(1 / 2, \pi_{E} \otimes \Omega\right)}{L(1, \pi, A d)} \hat{f}_{\mathfrak{p}}\left(\pi_{\mathfrak{p}}\right),
$$

is equal to

$$
\begin{aligned}
4\left|\Delta_{F}\right|^{\frac{3}{2}} L^{S(\Omega) \cup\{\mathfrak{p}\}}(1, \eta)\left(1+\delta\left(\Omega^{2}\right) \delta(\mathfrak{N}) \prod_{v \in \Sigma_{\infty}}(-1)^{k_{v}-1}\right) \int_{-2}^{2} \hat{f}_{\mathfrak{p}}(x) \mu_{\mathfrak{p}, E, \Omega} \\
-4 C\left(\boldsymbol{k}, \Omega, f_{\mathfrak{p}}\right) L^{S(\Omega)}(1, \eta)^{2} \frac{\sqrt{c(\Omega)\left|\Delta_{E}\right|}}{L\left(2,1_{F}\right) \sqrt{\left|\Delta_{F}\right|}} \prod_{v \mid \mathfrak{N}} \frac{1}{q_{v}-1} \prod_{v \in \Sigma_{\infty}} \frac{2 \pi}{2 k_{v}-1}
\end{aligned}
$$

where $\mathfrak{I}\left(f_{\mathfrak{p}}\right)$ is defined before Lemma 4.21, $C\left(\boldsymbol{k}, \Omega, f_{\mathfrak{p}}\right)$ is defined in Lemma 3.6 and the measure $\mu_{\mathfrak{p}, E, \Omega}$ is defined in Section 5.2.

We note in particular that when $f_{\mathfrak{p}}$ is the identity in $\mathcal{H}\left(G\left(F_{\mathfrak{p}}\right), Z_{\mathfrak{p}} R_{\mathfrak{p}}^{\times}\right)$the second term in the theorem above is equal to

$$
4\left|\Delta_{F}\right|^{\frac{3}{2}} L^{S(\Omega)}(1, \eta)\left(1+\delta\left(\Omega^{2}\right) \delta(\mathfrak{N}) \prod_{v \in \Sigma_{\infty}}(-1)^{k_{v}-1}\right) .
$$

We recall that by the Ramanujan conjecture $a_{\mathfrak{p}}(\pi) \in[-2,2]$ for all $\pi \in$ $\mathcal{F}(\mathfrak{N}, 2 \boldsymbol{k})$; see [Bla06] for the most general version. The distribution of the $\mathfrak{a}_{\mathfrak{p}}(\pi)$ has been considered by Sarnak [Sar87] and Serre [Ser97]. In [Ser97] it is proven, when $F=\mathbf{Q}$, that as $\mathfrak{N} \rightarrow \infty$ the set

$$
\left\{a_{\mathfrak{p}}(\pi): \pi \in \mathcal{F}(\mathfrak{N}, 2 \boldsymbol{k})\right\}
$$


becomes equidistributed with respect to the measure

$$
\mu_{\mathfrak{p}}=\frac{q_{\mathfrak{p}}+1}{\left(q_{\mathfrak{p}}^{\frac{1}{2}}+q_{\mathfrak{p}}^{-\frac{1}{2}}\right)^{2}-x^{2}} \frac{\sqrt{4-x^{2}}}{2 \pi} d x
$$

on $[-2,2]$. That is, for all $J \subset[-2,2]$,

$$
\lim _{\mathfrak{N} \rightarrow \infty} \frac{1}{\# \mathcal{F}(\mathfrak{N}, 2 \boldsymbol{k})} \sum_{\substack{\pi \in \mathcal{F}(\mathfrak{N}, 2 k) \\ a_{\mathfrak{p}}(\pi) \in J}} 1=\mu_{\mathfrak{p}}(J) .
$$

We note that $\mu_{\mathfrak{p}}$ comes from the spherical Plancherel measure on $\operatorname{PGL}\left(2, F_{\mathfrak{p}}\right)$.

Using Theorem 6.1 we obtain a variant of this equidistribution result where we include a weighting by $L^{\mathfrak{p}}\left(1 / 2, \pi_{E} \otimes \Omega\right)$.

Corollary 6.2. Let $J \subset[-2,+2]$. Then we have

$$
\lim _{|\mathfrak{N}| \rightarrow \infty} \frac{1}{|\mathfrak{N}|} \sum_{\substack{\pi \in \mathcal{F}(\mathfrak{N}, 2 \boldsymbol{k}) \\ a_{\mathfrak{p}}(\pi) \in J}} \frac{L^{\mathfrak{p}}\left(1 / 2, \pi_{E} \otimes \Omega\right)}{L^{\mathfrak{p}}(1, \pi, A d)}
$$

equal to

$$
4\left|\Delta_{F}\right|^{\frac{3}{2}} \frac{1}{2^{[F: \mathbf{Q}]}}\left(\begin{array}{c}
2 \boldsymbol{k}-2 \\
\boldsymbol{k}+\boldsymbol{m}-1
\end{array}\right)^{-1} L^{S(\Omega) \cup\{\mathfrak{p}\}}(1, \eta) L\left(2,1_{F_{\mathfrak{p}}}\right) \mu_{\mathfrak{p}}(J) .
$$

Here the limit is taken over squarefree ideals $\mathfrak{N}$ prime to $\mathfrak{c}(\Omega)$ and such that each prime dividing $\mathfrak{N}$ is inert and unramified in $E$ and the number of primes dividing $\mathfrak{N}$ has the same parity as $[F: \mathbf{Q}]$.

Proof. This result follows by an application of [Ser97, Proposition 2] to Theorem 6.1.

We note when $\Omega$ is trivial and $F=\mathbf{Q}$, we recover the main result of [RR05]. From this result we conclude the following.

Corollary 6.3. There are holomorphic cusp forms $\pi$ on $\mathrm{PGL}(2) / F$ of squarefree level and fixed weight such that $a_{\mathfrak{p}}(\pi)$ lies arbitrarily close to \pm 2 and $L\left(1 / 2, \pi_{E} \otimes\right.$ $\Omega) \neq 0$.

It is worth remarking that one could consider the average by normalizing by $|\mathcal{F}(\mathfrak{N}, 2 \boldsymbol{k})|$ rather than $|\mathfrak{N}|$. In order to have a finite limit with this normalization we need to add the technical restriction that $|\mathfrak{N}| \prod_{\mathfrak{p} \mid \mathfrak{N}}\left(1-\frac{1}{|\mathfrak{p}|}\right) \sim|\mathfrak{N}|$. For $F=\mathbf{Q}$ this condition reduces to $\varphi(N) \sim N$ where $\varphi$ is the Euler totient function. Using the well known fact that $|\mathcal{F}(N, 2 k)| \sim \frac{2 k-1}{12} \varphi(N)$ as $N \rightarrow \infty$ we get the following statement. 
Corollary 6.4. Let $F=\mathbf{Q}$ and $J \subset[-2,+2]$. Then

$$
\lim _{N \rightarrow \infty} \frac{1}{|\mathcal{F}(N, 2 k)|} \sum_{\substack{\pi \in \mathcal{F}(N, 2 k) \\ a_{p}(\pi) \in J}} \frac{L^{p}\left(1 / 2, \pi_{E} \otimes \Omega\right)}{L^{p}(1, \pi, A d)}
$$

is equal to

$$
\frac{24}{2 k-1}\left(\begin{array}{c}
2 k-2 \\
k+m-1
\end{array}\right)^{-1} L^{S(\Omega) \cup\{p\}}(1, \eta) L\left(2,1_{\mathbf{Q}_{p}}\right) \mu_{p}(J)
$$

where the limit is taken over squarefree $N$ such that $\varphi(N) \sim N$ and each prime dividing $N$ is inert and unramified in $E$ and does not divide $c(\Omega)$.

In principal, one could use the formulas developed in this paper to study the average computed by summing over the space of cusp forms and normalizing by the dimension of this space. Averaging in this way may remove the condition that $\varphi(N) \sim N$.

Finally, when $\Omega$ is unramified we have the following exact formula for all levels by combining Propositions 3.9 and 4.23.

Theorem 6.5. Let $E$ be a $C M$ extension of a totally real number field $F$. Let $\mathfrak{N} \subset \mathcal{O}_{F}$ be an ideal such that each prime dividing $\mathfrak{N}$ is unramified and inert in $E$ and such that the number of primes dividing $\mathfrak{N}$ has the same parity as $[F: \mathbf{Q}]$.

Let $\Omega: \mathbf{A}_{F}^{\times} E^{\times} \backslash \mathbf{A}_{E}^{\times} \rightarrow \mathbf{C}^{\times}$be a character which is everywhere unramified and such that for $v \in \Sigma_{\infty}$ the weight $m_{v}$ of $\Omega_{v}$ is strictly less than $k_{v}$. Assume furthermore that $E / F$ is unramified at the even places of $F$. Let $d \in \mathcal{O}_{F}$ be a generator of $\mathfrak{d}_{E / F}$. Then, with $C\left(\boldsymbol{k}, \Omega, \mathbf{1}_{\mathfrak{p}}\right)$ defined as in Lemma 3.6,x

$$
\begin{aligned}
\frac{1}{\sqrt{c(\Omega) d_{E / F}}|\mathfrak{N}|\left|\Delta_{F}\right|^{2}}\left(\begin{array}{c}
2 \boldsymbol{k}-2 \\
\boldsymbol{k}+\boldsymbol{m}-1
\end{array}\right) \sum_{\pi \in \mathcal{F}(\mathfrak{N}, 2 \boldsymbol{k})} \frac{L\left(1 / 2, \pi_{E} \otimes \Omega\right)}{L(1, \pi, A d)} \\
+C\left(\boldsymbol{k}, \Omega, \mathbf{1}_{\mathfrak{p}}\right) \frac{4 L(1, \eta)^{2}}{\left|\Delta_{F}\right|^{\frac{3}{2}} L\left(2,1_{F}\right)} \prod_{v \mid \mathfrak{N}} \frac{1}{q_{v}-1} \prod_{v \in \Sigma_{\infty}} \frac{\pi}{2 k_{v}-1},
\end{aligned}
$$

is equal to the sum of

$$
\frac{4 L(1, \eta) \sqrt{\left|\Delta_{F}\right|}}{2^{[F: \mathbf{Q}]} \sqrt{c(\Omega)} \sqrt{\left|\Delta_{E}\right|}}\left(1+\delta\left(\Omega^{2}\right) \delta(\mathfrak{N}) \prod_{v \in \Sigma_{\infty}}(-1)^{k_{v}-1}\right)
$$

and

$2 \frac{\left|\Delta_{F}\right|}{\left|\Delta_{E}\right|} \sum_{n}\left(\left|R_{E}\left(n \mathfrak{N}^{-1}\right)\right| \sigma\left(\mathfrak{d}_{E / F},(n+d)\right) \sum_{\mathfrak{a} \in R_{E}((n+d))} \Omega\left(\mathfrak{D}_{E / F}^{-1} \mathfrak{a}\right) \times \prod_{v \in \Sigma_{\infty}} P_{k_{v}, m_{v}}\left(\iota_{v}\left(\frac{n}{n+d}\right)\right)\right)$,

with the outer sum taken over the finite set of $n \in \mathfrak{N}$ such that

1. $\eta_{v}\left(1+\frac{d}{n}\right)=1$ for all $v \mid \mathfrak{d}_{E / F}$, and

2. $\iota_{v}(n)$ lies between $-\iota_{v}(d)$ and 0 for all $v \in \Sigma_{\infty}$. 


\subsection{Subconvexity}

We now apply our calculations of the relative trace formula to the problem of subconvexity.

Let $\pi_{1}$ and $\pi_{2}$ be cuspidal automorphic representations of $\operatorname{GL}\left(2, \mathbf{A}_{F}\right)$. The convexity bound for $L_{f i n}\left(1 / 2, \pi_{1} \times \pi_{2}\right)$ is that for $\epsilon>0$,

$$
L_{f i n}\left(1 / 2, \pi_{1} \times \pi_{2}\right) \ll_{\epsilon} C\left(\pi_{1} \times \pi_{2}\right)^{\frac{1}{4}+\epsilon},
$$

where $C\left(\pi_{1} \times \pi_{2}\right)=C_{f i n}\left(\pi_{1} \times \pi_{2}\right) C_{\infty}\left(\pi_{1} \times \pi_{2}\right)$ is the analytic conductor of $\pi_{1} \times \pi_{2}$; see [IS00a, Section 2.A]. $C_{f i n}\left(\pi_{1} \times \pi_{2}\right)$ is the conductor of $\pi_{1} \times \pi_{2}$ and $C_{\infty}\left(\pi_{1} \times \pi_{2}\right)$ depends only on the infinity types of $\pi_{1}$ and $\pi_{2}$; we refer to [IS00a, Section 2.A] for the precise definition. We note that when $\pi_{1}$ and $\pi_{2}$ have disjoint ramification $C_{f i n}\left(\pi_{1} \times \pi_{2}\right)=\left(C_{f i n}\left(\pi_{1}\right) C_{f i n}\left(\pi_{2}\right)\right)^{2}$.

The problem of beating the convex bound, with $\pi_{2}$ fixed, has been the study of many authors. When $F=\mathbf{Q}$ and $\pi_{1}$ and $\pi_{2}$ have trivial central character the convexity bound was beaten by Kowalski, Michel, and VanderKam [KMV02], with the central character condition being relaxed by Michel and Harcos [Mic04], [HM06]. In the case that $\Omega$ is trivial, so that the $L$-function factors as $L\left(s, \pi_{E}\right)=$ $L(s, \pi) L(s, \pi \otimes \eta)$, the convexity bound was beaten by Duke, Friedlander and Iwaniec [DFI94] in the level aspect over $\mathbf{Q}$ with $\pi$ fixed and $\eta$ varying. For number fields other than $\mathbf{Q}$ the first subconvex result was obtained by Cogdell, Piatetski-Shapiro and Sarnak [Cog03] in the case of a fixed Hilbert modular form twisted by a ray class character. Further extensions of these subconvexity results to cusp forms on arbitrary number fields have been obtained by Venkatesh [Ven05].

We now continue with the usual assumptions on $\pi \in \mathcal{F}(\mathfrak{N}, 2 \boldsymbol{k})$ and $\Omega$ as in Section 2 . We denote by $\sigma_{\Omega}$ the induction of $\Omega$ to an automorphic representation of $\operatorname{GL}\left(2, \mathbf{A}_{F}\right)$. Hence,

$$
L\left(s, \pi_{E} \otimes \Omega\right)=L\left(s, \pi \times \sigma_{\Omega}\right) .
$$

We have $C_{f i n}(\pi)=|\mathfrak{N}|$ and, by the formula for the conductor of an induced representation (see for example [Sch02, Section 1.2]), $C_{f i n}\left(\sigma_{\Omega}\right)=d_{E / F} c(\Omega)$. Thus, $C_{\text {fin }}\left(\pi \times \sigma_{\Omega}\right)=\left(|\mathfrak{N}| d_{E / F} c(\Omega)\right)^{2}$, and the convexity bound in the level aspect is given by

$$
L_{f i n}\left(1 / 2, \pi \times \sigma_{\Omega}\right) \ll_{k, \epsilon}\left(|\mathfrak{N}| d_{E / F} c(\Omega)\right)^{\frac{1}{2}+\epsilon} .
$$

We now proceed to apply our work to the problem of beating convexity for these $L$-functions. By combining our calculations of the relative trace formula, together with the bounds on the orbital integral integrals from Section 4.2.2, we will get an estimate for $L_{f i n}\left(1 / 2, \pi \times \sigma_{\Omega}\right)$ (Theorem 6.8 below) which beats the convexity bound as $\pi$ and $\Omega$ vary in a hybrid range.

The relative trace formula provides an expression for the first moment of $L\left(1 / 2, \pi \times \sigma_{\Omega}\right)$ averaged over $\pi \in \mathcal{F}(\mathfrak{N}, 2 \boldsymbol{k})$. The size of this family is approximately $|\mathfrak{N}|$ while the conductor of the $L$-function is $\left(|\mathfrak{N}| d_{E / F} c(\Omega)\right)^{2}$, this allows 
us to obtain estimates which beat convexity when $|\mathfrak{N}|$ is of size around $\sqrt{c(\Omega)}$. We note that over Q, Michel [Mic04] and Harcos-Michel [HM06] in their work on subconvexity for $L\left(s, \pi_{1} \times \pi_{2}\right)$ average over the family of modular forms of level $\left[C_{f i n}\left(\pi_{1}\right), C_{f i n}\left(\pi_{2}\right)\right]$ and bound the second moment of the $L$-function. By shortening the family we are able to get away with an estimate for only the first moment.

Recall that $\Omega$ is a unitary character on the idele class group of $E$ that is trivial when restricted to $\mathbf{A}_{F}^{\times}$, unramified above $\mathfrak{N}$ and has weight $\left|m_{v}\right|$ strictly less than $k_{v}$ for every infinite place $v$ of $F$. We let $S(\Omega)=\left\{\mathfrak{p}_{1}, \mathfrak{p}_{2}, \ldots, \mathfrak{p}_{m}\right\}$ denote the places of $F$ above which $\Omega$ is ramified, $\mathfrak{c}(\Omega)$ the norm of the conductor of $\Omega$ in $F$ and $c(\Omega)$ the absolute norm of $\mathfrak{c}(\Omega)$. Let $m=|S(\Omega)|, \mathfrak{c}(\Omega)=\prod_{i=1}^{m} \mathfrak{p}_{i}^{2 n_{i}}$ and $N_{F / \mathbf{Q}_{i}}=q_{i}$.

In the remainder of this section we fix $E$ and allow $\mathfrak{N}$ and $\Omega$ to vary. We recall that $D$ ramifies precisely at the infinite places of $F$ and the places dividing $\mathfrak{N}$. Therefore as $\mathfrak{N}$ varies, $D$ varies and hence the image of $E$ in $D$ depends on $\mathfrak{N}$. However $E$ as a field extension of $F$ does not depend on $\mathfrak{N}$. For the bounds we prove in this section, the $\ll_{E}$ notation refers to constants that only depend on $E$ as a field, such at $\sqrt{\left|\Delta_{E}\right|}$, and thus have no hidden dependence on $\mathfrak{N}$.

We begin with a necessary technical lemma which we will require in the bounding of the geometric expansion of $I(f)$. For $a \in F$ and integers $r_{i}, t_{i} \geq 0$ for $1 \leq i \leq m$ let

$$
S_{\left(r_{i}\right),\left(t_{i}\right)}(a)=\left\{y \in \mathfrak{N}: v_{\mathfrak{p}_{i}}(y)=r_{i}, v_{\mathfrak{p}_{i}}(y-a)=t_{i},\left|\iota_{v}(y)\right|<\left|\iota_{v}(a)\right|, i=1, \ldots, m, v \in \Sigma_{\infty}\right\} .
$$

Lemma 6.6. $S_{\left(r_{i}\right),\left(t_{i}\right)}(a)$ is empty unless for each $i=1, \ldots, m$,

$$
\begin{aligned}
& \text { 1. } r_{i}<v_{\mathfrak{p}_{i}}(a) \text { and } t_{i}=r_{i} \text { or } \\
& \text { 2. } r_{i}>v_{\mathfrak{p}_{i}}(a) \text { and } t_{i}=v_{\mathfrak{p}_{i}}(a) \text { or } \\
& \text { 3. } r_{i}=v_{\mathfrak{p}_{i}}(a) \text { and } t_{i} \geq v_{\mathfrak{p}_{i}}(a) \text {. }
\end{aligned}
$$

In addition,

$$
\left|S_{\left(r_{i}\right),\left(t_{i}\right)}(a)\right| \leq \frac{c(F)\left|N_{F / \mathbf{Q}}(a)\right|}{\left|N_{F / \mathbf{Q}}\left(\mathfrak{N p}_{1}^{\max \left\{r_{1}, t_{1}\right\}} \ldots \mathfrak{p}_{m}^{\max \left\{r_{m}, t_{m}\right\}}\right)\right|}
$$

where $c(F)$ is a constant that only depends on $F$.

Proof. It is clear that $S_{\left(r_{i}\right),\left(t_{i}\right)}(a)$ is empty unless conditions 1,2 or 3 hold for each $i$. We now proceed to prove the bound following the ideas of the proof in [Lan94, §V.1, Theorem 0].

Since $S_{\left(r_{i}\right),\left(t_{i}\right)}(a)$ is finite there exists an integer $n$ such that

$$
n<\left|S_{\left(r_{i}\right),\left(t_{i}\right)}(a)\right| \leq n+1 .
$$

Pick a $v_{0} \in \Sigma_{\infty}$. Identify $F_{v_{0}}$ with the real line. By the condition $\left|\iota_{v_{0}}(y)\right|<$ $\left|\iota_{v_{0}}(a)\right|, S_{\left(r_{i}\right),\left(t_{i}\right)}(a)$ is contained in the interval centered at the origin of length $2\left|\iota_{v_{0}}(a)\right|$. By the Pigeon Hole Principle if we divide this interval into $n$ equal 
subintervals there must be at least two distinct elements $x, y \in S_{\left(r_{i}\right),\left(t_{i}\right)}(a)$ such that $x$ and $y$ are in the same subinterval. Thus

$$
\left|\iota_{v_{0}}(x-y)\right| \leq \frac{2\left|\iota_{v_{0}}(a)\right|}{n} .
$$

For all $v \in \Sigma_{\infty}$ with $v$ not equal to $v_{0}$,

$$
\left|\iota_{v}(x-y)\right| \leq 2\left|\iota_{v}(a)\right| .
$$

We also know that

$$
|x-y|_{\mathfrak{p}_{i}} \leq q_{i}^{-r_{i}}
$$

and

$$
|x-y|_{\mathfrak{p}_{i}}=|(x-a)-(y-a)|_{\mathfrak{p}_{i}} \leq q_{i}^{-t_{i}} .
$$

Thus

$$
|x-y|_{\mathfrak{p}_{i}} \leq q_{i}^{-\max \left\{r_{i}, t_{i}\right\}}
$$

For $v \mid \mathfrak{N}$,

$$
|x-y|_{v} \leq|\mathfrak{N}|_{v}
$$

Thus

$$
1=\prod_{v}|x-y|_{v} \leq \frac{2^{[F: \mathbf{Q}]}\left|N_{F / \mathbf{Q}}(a)\right|}{n\left|N_{F / \mathbf{Q}}\left(\mathfrak{N p}_{1}^{\max \left\{r_{1}, t_{1}\right\}} \ldots \mathfrak{p}_{m}^{\max \left\{r_{m}, t_{m}\right\}}\right)\right|} .
$$

Hence

$$
\left|S_{\left(r_{i}\right),\left(t_{i}\right)}(a)\right| \leq n+1 \leq 2 n \leq \frac{2^{[F: \mathbf{Q}]+1}\left|N_{F / \mathbf{Q}}(a)\right|}{\left|N_{F / \mathbf{Q}}\left(\mathfrak{N P}_{1}^{\max \left\{r_{1}, t_{1}\right\}} \ldots \mathfrak{p}_{m}^{\max \left\{r_{m}, t_{m}\right\}}\right)\right|}
$$

We now proceed to bound the regular terms in the trace formula. By Lemma 4.21

$$
I_{\text {reg }}(f)=\sum_{\xi \in S\left(\Omega, \mathfrak{N}, 1_{\mathfrak{p}}\right)} I(\xi, f)
$$

where $S\left(\Omega, \mathfrak{N}, 1_{\mathfrak{p}}\right)$ is the set of $\xi \in \epsilon N E^{\times}$such that $v(\xi) \geq 1$ for all $v \mid \mathfrak{N}$ and $(1-\xi)^{-1} \in\left(\mathfrak{c}(\Omega) \mathfrak{d}_{E / F}\right)^{-1}$. The set $S\left(\Omega, \mathfrak{N}, 1_{\mathfrak{p}}\right)$ is approximately of size $\frac{c(\Omega) d_{E / F}}{|\mathfrak{N}|}$. However in the following Lemma we can take advantage of the bounds on the local orbital integrals given in Lemma 4.19, which improve as $c(\Omega)$ increases, to get a good bound on $I_{\text {reg }}(f)$.

Lemma 6.7. Assume that $F, E$ and $\boldsymbol{k}$ are fixed and $f_{\mathfrak{p}}=\mathbf{1}_{\mathfrak{p}}$. Then for all $\epsilon>0$

$$
\left|I_{\text {reg }}(f)\right| \ll_{F, E, k, \epsilon} \frac{c(\Omega)^{\epsilon}}{|\mathfrak{N}|} .
$$


Proof. Let $\mathfrak{A}_{1}, \ldots, \mathfrak{A}_{h_{F}}$ be a fixed set of representatives of the ideal classes of $F$. Then for any ideal $\mathfrak{I}$ of $\mathcal{O}_{F}$ there exists an $i$ such that $\mathfrak{A}_{i} \mathfrak{I}$ is principal. Let $a \in F$ be such that

$$
a \mathcal{O}_{F}=\mathfrak{A}_{i} \mathfrak{c}(\Omega) \mathfrak{d}_{E / F}
$$

for some $i$. Then

$$
\left|\frac{N_{F / \mathbf{Q}}(a)}{c(\Omega) d_{E / F}}\right| \leq c(F)=\max \left\{\left|\mathfrak{A}_{i}\right|: 1 \leq i \leq h_{F}\right\} .
$$

For $\xi \in S\left(\Omega, \mathfrak{N}, 1_{\mathfrak{p}}\right)$ we have,

$$
\xi=\xi_{y}:=\frac{y}{y-a},
$$

for some $y \in \mathfrak{N}$ such that $\left|\iota_{v}(y)\right|<\left|\iota_{v}(a)\right|$ for all $v \in \Sigma_{\infty}$. Hence we can partition these $y$ into the sets $S_{\left(r_{i}\right),\left(t_{i}\right)}(a)$. Thus

$$
I_{\text {reg }}(f)=\sum_{r_{1} \geq 0} \sum_{r_{2} \geq 0} \ldots \sum_{r_{m} \geq 0} \sum_{t_{1} \geq 0} \sum_{t_{2} \geq 0} \ldots \sum_{t_{m} \geq 0} \sum_{y \in S_{\left(r_{i}\right),\left(t_{i}\right)}(a)} I\left(\xi_{y}, f^{S(\Omega)}\right) \prod_{i=1}^{m} I\left(\xi_{y}, f_{\mathfrak{p}_{i}}\right) .
$$

First we consider the integrals $I\left(\xi_{y}, f^{S(\Omega)}\right)$. For an ideal $\mathfrak{a} \subset \mathcal{O}_{F}$ we define $R_{E}^{S(\Omega)}(\mathfrak{a})=\left\{\mathfrak{b} \subset \mathcal{O}_{E}: N_{E_{v} / F_{v}}\left(\mathfrak{b} \mathcal{O}_{E_{v}}\right)=\mathfrak{a} \mathcal{O}_{F_{v}}\right.$ for $v \notin S(\Omega)$ and $\mathfrak{p}_{v} \nmid \mathfrak{b}$ for $\left.v \in S(\Omega)\right\}$.

By the proof of Proposition 4.23 we see that

$$
\left|I\left(\xi_{y}, f^{S(\Omega)}\right)\right| \leq C(E)\left|R_{E}^{S(\Omega)}\left(y \mathfrak{N}^{-1}\right)\right|\left|R_{E}^{S(\Omega)}((y-a))\right|\left|I\left(\xi, f_{\Sigma_{\infty}}\right)\right|,
$$

where $C(E)$ is a constant depending only on $E$. Furthermore, from Lemma 4.14 , it is clear that

$$
\left|I\left(\xi_{y}, f_{\Sigma_{\infty}}\right)\right| \leq C(\boldsymbol{k}),
$$

where $C(\boldsymbol{k})$ is a constant depending only on $\boldsymbol{k}$.

Let $\sigma_{0}(x)$ denote the number of divisors of $x$. For any ideal $\mathfrak{a} \in \mathcal{O}_{F}$,

$$
\left|R_{E}^{S(\Omega)}(\mathfrak{a})\right| \leq \sigma_{0}\left(N_{F / \mathbf{Q}}(\mathfrak{a})\right)^{[F: \mathbf{Q}]} \ll_{\epsilon}\left|N_{F / \mathbf{Q}}(\mathfrak{a})\right|^{\epsilon} .
$$

For any $y \in S_{\left(r_{i}\right),\left(t_{i}\right)}(a),\left|\iota_{v}(y)\right|<\left|\iota_{v}(a)\right|$ and $\left|\iota_{v}(y-a)\right|<\left|\iota_{v}(a)\right|$ for all $v \in \Sigma_{\infty}$. Thus $\left|N_{F / \mathbf{Q}}(y)\right|=\prod_{v \in \Sigma_{\infty}}\left|\iota_{v}(y)\right| \leq\left|N_{F / \mathbf{Q}}(a)\right|$ and similarly $\left|N_{F / \mathbf{Q}}(y-a)\right| \leq$ $\left|N_{F / \mathbf{Q}}(a)\right|$.

By (7), (8) and (9),

$$
\left|I\left(\xi_{y}, f^{S(\Omega)}\right)\right| \ll_{F, E, \boldsymbol{k}, \epsilon}\left|N_{F / \mathbf{Q}}(a)\right|^{\epsilon} .
$$


Combining (6) and (10) we have,

$$
\left|I_{r e g}(f)\right| \ll_{F, E, k, \epsilon}\left|N_{F / \mathbf{Q}} a\right|^{\epsilon} \sum_{r_{1} \geq 0} \sum_{r_{2} \geq 0} \ldots \sum_{r_{m} \geq 0} \sum_{t_{1} \geq 0} \sum_{t_{2} \geq 0} \ldots \sum_{t_{m} \geq 0} \sum_{y \in S_{\left(r_{i}\right),\left(t_{i}\right)}(a)} \prod_{i=1}^{m}\left|I\left(\xi_{y}, f_{\mathfrak{p}_{i}}\right)\right| .
$$

To bound $\left|I\left(\xi_{y}, f_{\mathfrak{p}_{i}}\right)\right|$ we first note that

$$
v_{\mathfrak{p}_{i}}(1-\xi)=v_{\mathfrak{p}_{i}}(a)-v_{\mathfrak{p}_{i}}(y-a) .
$$

Thus by Lemma 4.19 , for $y \in S_{\left(r_{i}\right),\left(t_{i}\right)}(a)$,

$$
\left|I\left(\xi_{y}, f_{\mathfrak{p}_{i}}\right)\right| \leq \begin{cases}C\left(E_{v}, F_{v}\right) q_{i}^{-n_{i}} L\left(1, \eta_{v}\right)^{2} q_{i}^{\frac{r_{i}-v_{\mathfrak{p}_{i}}(a)}{2}} & 0 \leq r_{i}<v_{\mathfrak{p}_{i}}(a) \\ C\left(E_{v}, F_{v}\right) q_{i}^{-n_{i}} L\left(1, \eta_{v}\right)\left(1+t_{i}-r_{i}\right) & r_{i}=v_{\mathfrak{p}_{i}}(a) \\ C\left(E_{v}, F_{v}\right) q_{i}^{-n_{i}} L\left(1, \eta_{v}\right)\left(1+r_{i}-v_{\mathfrak{p}_{i}}(a)\right) & r_{i}>v_{\mathfrak{p}_{i}}(a)\end{cases}
$$

where $C\left(E_{v}, F_{v}\right)$ is the constant in the statement of Lemma 4.19. In particular, $C\left(E_{v}, F_{v}\right)=1$ if $v$ is unramified in $E$.

Because the bounds in Lemma 6.6 and (12) depend only on $r_{i}$ and $t_{i}$ for each $i$,

$$
\left|I_{r e g}(f)\right| \ll_{F, E, k, \epsilon}\left|N_{F / \mathbf{Q}}(a)\right|^{\epsilon} \frac{\left|N_{F / \mathbf{Q}}(a)\right|}{\left|N_{F / \mathbf{Q}} \mathfrak{N}\right|} \prod_{i=1}^{m} \sum_{r_{i} \geq 0} \sum_{t_{i} \geq 0} q_{i}^{-\max \left\{r_{i}, t_{i}\right\}}\left|I\left(\xi_{y_{r_{i}, t_{i}}}, f_{\mathfrak{p}_{i}}\right)\right|,
$$

where we choose $y_{r_{i}, t_{i}}$ to be any element in $F_{\mathfrak{p}_{i}}$ such that $v_{\mathfrak{p}_{i}}\left(y_{r_{i}, t_{i}}\right)=r_{i}$ and $v_{\mathfrak{p}_{i}}\left(y_{r_{i}, t_{i}}-a\right)=t_{i}$.

Again by Lemma 6.6 and (12), for $i$ fixed

$$
\begin{aligned}
& \sum_{r_{i} \geq 0} \sum_{t_{i} \geq 0} q_{i}^{-\max \left\{r_{i}, t_{i}\right\}}\left|I\left(\xi_{y_{r_{i}, t_{i}}}, f_{\mathfrak{p}_{i}}\right)\right| \\
& \leq \sum_{v_{\mathfrak{p}_{i}}(a)>r_{i} \geq 0} C\left(E_{v}, F_{v}\right) q_{i}^{-n_{i}} L\left(1, \eta_{v}\right)^{2} q_{i}^{\frac{r_{i}-v_{\mathfrak{p}_{i}}(a)}{2}} q_{i}^{-r_{i}} \\
& +\sum_{t_{i} \geq v_{\mathfrak{p}_{i}}(a)} C\left(E_{v}, F_{v}\right) q_{i}^{-n_{i}} L\left(1, \eta_{v}\right)\left(1+t_{i}-v_{\mathfrak{p}_{i}}(a)\right) q_{i}^{-t_{i}}+\sum_{r_{i}>v_{\mathfrak{p}_{i}}(a)} C\left(E_{v}, F_{v}\right) q_{i}^{-n_{i}} L\left(1, \eta_{v}\right)\left(1+r_{i}-v_{\mathfrak{p}_{i}}(a)\right) q_{i}^{-r_{i}} \\
& \leq \sum_{r_{i} \geq 0} C\left(E_{v}, F_{v}\right) q_{i}^{-n_{i}} L\left(1, \eta_{v}\right)^{2} q_{i}^{\frac{r_{i}-v_{\mathfrak{p}_{i}}(a)}{2}} q_{i}^{-r_{i}}+2 \sum_{\ell \geq v_{\mathfrak{p}_{i}}(a)} C\left(E_{v}, F_{v}\right) q_{i}^{-n_{i}} L\left(1, \eta_{v}\right)\left(1+\ell-v_{\mathfrak{p}_{i}}(a)\right) q_{i}^{-\ell} \\
& \leq C\left(E_{v}, F_{v}\right) L\left(1, \eta_{v}\right) q_{i}^{-n_{i}-\frac{v_{\mathfrak{p}_{i}}(a)}{2}}\left[\frac{L\left(1, \eta_{v}\right)}{1-q_{i}^{-1 / 2}}+\frac{2 q_{i}^{\frac{-v_{\mathfrak{p}_{i}}(a)}{2}}}{\left(1-q_{i}^{-1}\right)^{2}}\right] \\
& \leq 2^{5} q_{i}^{-n_{i}-\frac{v_{\mathfrak{p}_{i}}(a)}{2}} C\left(E_{v}, F_{v}\right) \\
& \leq 2^{5} q_{i}^{-2 n_{i}} C\left(E_{v}, F_{v}\right) \text {. }
\end{aligned}
$$


Finally we can bound the product of these terms over $1 \leq i \leq m$. We note that

$$
2^{|S(\Omega)|} \leq \sigma_{0}(c(\Omega))^{[F: \mathbf{Q}]} \ll_{\epsilon} c(\Omega)^{\epsilon} .
$$

Also, because $C\left(E_{v}, F_{v}\right)=1$ unless $v$ ramifies in $E$,

$$
\prod_{i=1}^{m} C\left(E_{v}, F_{v}\right) \ll_{E, F} 1 .
$$

By these facts,

$$
\prod_{i=1}^{m} \sum_{r_{i}} \sum_{t_{i}} q_{i}^{-\max \left\{r_{i}, t_{i}\right\}}\left|I\left(\xi_{y}, f_{\mathfrak{p}_{i}}\right)\right| \ll_{\epsilon, F, E} \frac{c(\Omega)^{\epsilon}}{c(\Omega)} .
$$

Combining (13) and (14) and applying (5) we conclude

$$
\left|I_{r e g}(f)\right| \ll_{F, E, k, \epsilon} \frac{c(\Omega)^{\epsilon}}{|\mathfrak{N}|} .
$$

Finally we combine the spectral expansion for $I(f)$, Proposition 3.9, together with the calculation of $I_{\text {irreg }}(f)$, Proposition 4.20, with the bound on $I_{\text {reg }}(f)$ established above. In the theorem below the term $|\mathfrak{N}|^{1+\epsilon} c(\Omega)^{\epsilon}$ comes from the irregular term and $|\mathfrak{N}|^{\epsilon} c(\Omega)^{\frac{1}{2}+\epsilon}$ comes from the bounds on the regular orbital integrals.

Theorem 6.8. Fix a totally real number field $F$ and a $C M$ extension $E$ of $F$. Let $\mathfrak{N}$ be a squarefree ideal in $\mathcal{O}_{F}$ such that the number of primes dividing $\mathfrak{N}$ has the same parity as $[F: \mathbf{Q}]$ and such that each prime of $F$ dividing $\mathfrak{N}$ is inert and unramified in $E$. Let $\Omega$ be a character of $\mathbf{A}_{F}^{\times} E^{\times} \backslash \mathbf{A}_{E}^{\times}$which is unramified above $\mathfrak{N}$ and has weights at the archimedean places strictly less than $\boldsymbol{k}$. Then for any $\epsilon>0$,

$$
L_{\text {fin }}\left(1 / 2, \pi \times \sigma_{\Omega}\right) \ll_{F, E, k, \epsilon}|\mathfrak{N}|^{1+\epsilon} c(\Omega)^{\epsilon}+|\mathfrak{N}|^{\epsilon} c(\Omega)^{\frac{1}{2}+\epsilon},
$$

for all $\pi \in \mathcal{F}(\mathfrak{N}, 2 \boldsymbol{k})$.

Proof. We note that with the weight $\boldsymbol{k}$ fixed there are only finitely many possibilities for the archimedean type of $\Omega$, hence it suffices to prove the same bound for the completed $L$-function.

We take $f_{\mathfrak{p}}=\mathbf{1}_{\mathfrak{p}}$ then from the spectral expansion for $I(f)$ we have, by Proposition 3.9,

$$
I(f) \geq \frac{L_{S(\Omega)}(1, \eta)^{2}}{2\left|\Delta_{F}\right|^{2} \sqrt{d_{E / F} c(\Omega)}} \frac{4^{[F: \mathbf{Q}]}}{|\mathfrak{N}|}\left(\begin{array}{c}
2 \boldsymbol{k}-2 \\
\boldsymbol{k}+\boldsymbol{m}-1
\end{array}\right) \sum_{\pi \in \mathcal{F}(\mathfrak{N}, 2 \boldsymbol{k})} \frac{L\left(1 / 2, \pi_{E} \otimes \Omega\right)}{L(1, \pi, A d)} .
$$


On the geometric side, we recall that we have written

$$
I(f)=I_{\text {irreg }}(f)+I_{\text {reg }}(f)
$$

in Section 4.3. By Proposition 4.20 we have,

$$
\left|I_{\text {irreg }}(f)\right| \ll_{F, E, \epsilon} \frac{c(\Omega)^{\epsilon}}{\sqrt{c(\Omega)}},
$$

and by Lemma 6.7 we have, for all $\epsilon>0$,

$$
\left|I_{\text {reg }}(f)\right| \ll_{F, E, k, \epsilon} \frac{c(\Omega)^{\epsilon}}{|\mathfrak{N}|} .
$$

Hence, combining (15), (16), (17) and (18), and noting that

$$
\frac{1}{L_{S(\Omega)}(1, \eta)} \leq 2^{2|S(\Omega)|} \leq \sigma_{0}(c(\Omega))^{2[F: \mathbf{Q}]} \ll_{\epsilon} c(\Omega)^{\epsilon},
$$

we get,

$$
\sum_{\pi \in \mathcal{F}(\mathfrak{N}, 2 k)} \frac{L\left(1 / 2, \pi_{E} \otimes \Omega\right)}{L(1, \pi, A d)} \ll_{F, E, \boldsymbol{k}, \epsilon}|\mathfrak{N}| c(\Omega)^{\epsilon}+c(\Omega)^{\frac{1}{2}+\epsilon} .
$$

Now using positivity of $L\left(1 / 2, \pi_{E} \otimes \Omega\right)$, which is clear from the period formula, we have

$$
\frac{L\left(1 / 2, \pi_{E} \otimes \Omega\right)}{L(1, \pi, A d)} \leq \sum_{\pi \in \mathcal{F}(\mathfrak{N}, 2 k)} \frac{L\left(1 / 2, \pi_{E} \otimes \Omega\right)}{L(1, \pi, A d)}
$$

for any $\pi \in \mathcal{F}(\mathfrak{N}, 2 \boldsymbol{k})$. We now use that for all $\epsilon>0$,

$$
L(1, \pi, A d) \ll_{k, \epsilon}|\mathfrak{N}|^{\epsilon} ;
$$

cf [IK04, Theorem 5.41]. Hence by (19), (20) and (21) we have, for all $\epsilon>0$, $L\left(1 / 2, \pi_{E} \otimes \Omega\right) \ll_{k, \epsilon}|\mathfrak{N}|^{\epsilon} \frac{L\left(1 / 2, \pi_{E} \otimes \Omega\right)}{L(1, \pi, A d)} \ll_{F, E, k, \epsilon}|\mathfrak{N}|^{1+\epsilon} c(\Omega)^{\epsilon}+|\mathfrak{N}|^{\epsilon} c(\Omega)^{\frac{1}{2}+\epsilon}$.

Finally we explicate how this Theorem gives a subconvex bound as $\mathfrak{N}$ and $\Omega$ vary in certain ranges.

Corollary 6.9. For $0 \leq t<\frac{1}{6}$ and $\epsilon>0$,

$$
L_{f i n}\left(1 / 2, \pi \times \sigma_{\Omega}\right) \ll_{F, E, \boldsymbol{k}, \epsilon}(c(\Omega)|\mathfrak{N}|)^{\frac{1}{2}-t},
$$

for $\pi \in \mathcal{F}(\mathfrak{N}, 2 \boldsymbol{k})$; with $\mathfrak{N}$ and $\Omega$ satisfying the conditions of Theorem 6.8 and

$$
c(\Omega)^{\frac{2 t+\epsilon}{1-(2 t+\epsilon)}} \leq|\mathfrak{N}| \leq c(\Omega)^{\frac{1-(2 t+\epsilon)}{1+2 t+\epsilon}} .
$$


Proof. To beat convexity we need,

$$
|\mathfrak{N}|^{1+\epsilon} c(\Omega)^{\epsilon} \leq|\mathfrak{N}|^{\frac{1}{2}-t} c(\Omega)^{\frac{1}{2}-t}
$$

and

$$
|\mathfrak{N}|^{\epsilon} c(\Omega)^{\frac{1}{2}+\epsilon} \leq|\mathfrak{N}|^{\frac{1}{2}-t} c(\Omega)^{\frac{1}{2}-t}
$$

Thus we need

$$
c(\Omega)^{\frac{2 \epsilon+2 t}{1-2 t-2 \epsilon}} \leq|\mathfrak{N}| \leq c(\Omega)^{\frac{1-2 t-2 \epsilon}{1+2 t+2 \epsilon}} .
$$

We note that the range for $|\mathfrak{N}|$ is non-empty provided $\frac{1}{6}>t \geq 0$.

\subsection{Classical reformulation}

To finish we work out Theorem 6.1 in the case $F=\mathbf{Q}$ classically. We fix an imaginary quadratic field $E=\mathbf{Q}(\sqrt{-d})$ of discriminant $-d$ and let $\chi_{-d}=\left(\frac{-d}{\cdot}\right)$ denote the associated quadratic Dirichlet character.

Let $N$ be a squarefree integer which is the product of an odd number of primes $p$ satisfying $\chi_{-d}(p)=-1$. For a positive integer $k$ we denote by $\mathcal{F}(N, 2 k)$ the finite set of normalized newforms of level $N$, weight $2 k$, trivial nebentypus and which are eigenforms for all the Hecke operators. On $\mathcal{F}(N, 2 k)$ we take the Petersson inner product defined by

$$
(f, f)=\int_{\Gamma_{0}(N) \backslash \mathcal{H}}|f(x+i y)|^{2} y^{2 k} \frac{d x d y}{y^{2}} .
$$

We note that,

$$
L\left(1, \pi_{f}, A d\right)=\frac{2^{2 k}}{N}(f, f)
$$

where $\pi_{f}$ denotes the automorphic representation of $\mathrm{GL}\left(2, \mathbf{A}_{\mathbf{Q}}\right)$ generated by $f$. We denote by $L(s, f)$ the completed $L$-function of $f$, which has a functional equation relating the value at $s$ to $2 k-s$.

We now fix a character $\Omega: E^{\times} \backslash \mathbf{A}_{E}^{\times} \rightarrow \mathbf{C}$ whose restriction to $\mathbf{A}_{\mathbf{Q}}^{\times}$is trivial. At infinity we have

$$
\Omega_{\infty}: z \mapsto\left(\frac{z}{\bar{z}}\right)^{m},
$$

with $m \in \mathbf{Z}$. We recall that when $\Omega$ does not factor through the norm map $N: \mathbf{A}_{E}^{\times} \rightarrow \mathbf{A}_{\mathbf{Q}}^{\times}$there is a modular form $g_{\Omega}$ of level $d c(\Omega)$, weight $2|m|+1$ and nebentypus $\chi_{-d}$ such that

$$
L\left(s, g_{\Omega}\right)=L(s, \Omega)
$$

For $f \in \mathcal{F}(N, 2 k)$ we let $L\left(s, f \times g_{\Omega}\right)$ denote the completed Rankin-Selberg $L$ function which satisfies a functional equation relating the value at $s$ to $2 k+$ $2|m|+1-s$.

We recall the well known facts that for the completed $L$-functions, $L\left(2,1_{\mathbf{Q}}\right)=$ $\frac{\pi}{6}$ and

$$
L\left(1, \chi_{-d}\right)=\frac{h_{-d}}{u_{-d} \sqrt{d}}
$$


where $h_{-d}$ denotes the class number of $E$ and $u_{-d}=\# \mathcal{O}_{E}^{\times} /\{ \pm 1\}$.

Taking into account that the gamma factor for $L(k, f)$ is $2(2 \pi)^{-k} \Gamma(k)$ and the gamma factor for $L\left(k+|m|+\frac{1}{2}, f \times g_{\Omega}\right)$ is $4(2 \pi)^{-2 k} \Gamma(k+m) \Gamma(k-m)$, we now apply Theorem 6.1 to get the following following averages for the finite parts of the $L$-functions.

Theorem 6.10. Let $N$ be a squarefree integer as above with $N>d$. Then we have

$$
\frac{u_{-d} \sqrt{d}}{8 \pi^{2}} \sum_{f \in \mathcal{F}(N, 2)} \frac{L_{f i n}(1, f) L_{f i n}\left(1, f \otimes \chi_{-d}\right)}{(f, f)}=h_{-d}\left(1-\frac{12 h_{-d}}{u_{-d} \varphi(N)}\right),
$$

where $\varphi$ denotes the Euler totient function. When $k>1$ we have

$$
\frac{(2 k-2) ! u_{-d} \sqrt{d}}{2 \pi(4 \pi)^{2 k-1}} \sum_{f \in \mathcal{F}(N, 2 k)} \frac{L_{f i n}(k, f) L_{f i n}\left(k, f \otimes \chi_{-d}\right)}{(f, f)}=h_{-d} .
$$

For a character $\Omega$ as above which does not factor through the norm we get for $N>d c(\Omega)$,

$$
\frac{(2 k-2) ! u_{-d} \sqrt{d} L_{S(\Omega)}\left(1, \chi_{-d}\right)}{2 \pi(4 \pi)^{2 k-1}} \sum_{f \in \mathcal{F}(N, 2 k)} \frac{L_{f i n}\left(k+|m|+\frac{1}{2}, f \times g_{\Omega}\right)}{(f, f)}=h_{-d} .
$$

We note that when the level is prime the first part of this Theorem agrees with Duke's asymptotic result [Duk95, Proposition 2] and the first and second parts agree with Michel and Ramakrishnan's exact formula [MR]. One can see [MR, p.5] for explicit examples verifying that this formula agrees with known data.

\section{References}

[Bla06] Don Blasius, Hilbert modular forms and the Ramanujan conjecture, Noncommutative geometry and number theory, Aspects Math., E37, Vieweg, Wiesbaden, 2006, pp. 35-56. MR MR2327298

[Cog03] James W. Cogdell, On sums of three squares, J. Théor. Nombres Bordeaux 15 (2003), no. 1, 33-44, Les XXIIèmes Journées Arithmetiques (Lille, 2001). MR MR2018999 (2005d:11072)

[DFI94] W. Duke, J. B. Friedlander, and H. Iwaniec, Bounds for automorphic L-functions. II, Invent. Math. 115 (1994), no. 2, 219-239. MR MR1258904 (95a:11044)

[Duk95] William Duke, The critical order of vanishing of automorphic Lfunctions with large level, Invent. Math. 119 (1995), no. 1, 165-174. MR MR1309975 (95k:11075) 
[GP91] Benedict H. Gross and Dipendra Prasad, Test vectors for linear forms, Math. Ann. 291 (1991), no. 2, 343-355. MR MR1129372 (92k:22028)

[Gro87] Benedict H. Gross, Heights and the special values of L-series, Number theory (Montreal, Que., 1985), CMS Conf. Proc., vol. 7, Amer. Math. Soc., Providence, RI, 1987, pp. 115-187. MR MR894322 (89c:11082)

[HM06] Gergely Harcos and Philippe Michel, The subconvexity problem for Rankin-Selberg L-functions and equidistribution of Heegner points. II, Invent. Math. 163 (2006), no. 3, 581-655. MR MR2207235

[IK04] Henryk Iwaniec and Emmanuel Kowalski, Analytic number theory, American Mathematical Society Colloquium Publications, vol. 53, American Mathematical Society, Providence, RI, 2004. MR MR2061214 (2005h:11005)

[ILS00] Henryk Iwaniec, Wenzhi Luo, and Peter Sarnak, Low lying zeros of families of L-functions, Inst. Hautes Études Sci. Publ. Math. (2000), no. 91, 55-131 (2001). MR MR1828743 (2002h:11081)

[IS00a] H. Iwaniec and P. Sarnak, Perspectives on the analytic theory of L-functions, Geom. Funct. Anal. (2000), no. Special Volume, Part II, 705-741, GAFA 2000 (Tel Aviv, 1999). MR MR1826269 (2002b:11117)

[IS00b] Henryk Iwaniec and Peter Sarnak, The non-vanishing of central values of automorphic L-functions and Landau-Siegel zeros, Israel J. Math. 120 (2000), no. , part A, 155-177. MR MR1815374 (2002b:11115)

[Jac86] Hervé Jacquet, Sur un résultat de Waldspurger, Ann. Sci. Éc. Norm. Supér., IV. 19 (1986), no. 2, 185-229.

[JC01] Hervé Jacquet and Nan Chen, Positivity of quadratic base change L-functions, Bull. Soc. Math. France 129 (2001), no. 1, 33-90. MR MR1871978 (2003b:11048)

[JL70] Hervé Jacquet and Robert P. Langlands, Automorphic forms on GL(2), Springer-Verlag, Berlin, 1970, Lecture Notes in Mathematics, Vol. 114. MR MR0401654 (53 \#5481)

[KMV02] E. Kowalski, P. Michel, and J. VanderKam, Rankin-Selberg Lfunctions in the level aspect, Duke Math. J. 114 (2002), no. 1, 123191. MR MR1915038 (2004c:11070)

[Lan94] Serge Lang, Algebraic number theory, second ed., Graduate Texts in Mathematics, vol. 110, Springer-Verlag, New York, 1994. MR MR1282723 (95f:11085)

[Mic04] Phillipe Michel, The subconvexity problem for Rankin-Selberg Lfunctions and equidistribution of Heegner points, Ann. of Math. (2) 160 (2004), no. 1, 185-236. MR MR2119720 (2006d:11048) 
[MR] Philippe Michel and Dinakar Ramakrishnan, Consequences of the Gross/Zagier formulae: stability of average L-values, subconvexity and non-vanishing $\bmod p$, to appear in the memorial volume for Serge Lang, Springer-Verlag (2009).

[MV07] Philippe Michel and Akshay Venkatesh, Heegner points and nonvanishing of Rankin/Selberg L-functions, Analytic number theory, Clay Math. Proc., vol. 7, Amer. Math. Soc., Providence, RI, 2007, pp. 169-183. MR MR2362200

[MW09] Kimball Martin and David Whitehouse, Central L-values and toric periods for GL(2), Int. Math. Res. Not. IMRN (2009), no. 1, Art. ID rnn127, 141-191. MR MR2471298

[Pop06] Alexandru A. Popa, Central values of Rankin L-series over real quadratic fields, Compos. Math. 142 (2006), no. 4, 811-866. MR MR2249532

[Roy00] Emmanuel Royer, Facteurs $\mathbf{Q}$-simples de $J_{0}(N)$ de grande dimension et de grand rang, Bull. Soc. Math. France 128 (2000), no. 2, 219-248. MR MR1772442 (2001j:11041)

[RR05] Dinakar Ramakrishnan and Jonathan Rogawski, Average values of modular L-series via the relative trace formula, Pure Appl. Math. Q. 1 (2005), no. 4, 701-735. MR MR2200997

[Sar87] Peter Sarnak, Statistical properties of eigenvalues of the Hecke operators, Analytic number theory and Diophantine problems (Stillwater, OK, 1984), Progr. Math., vol. 70, Birkhäuser Boston, Boston, MA, 1987, pp. 321-331. MR MR1018385 (90k:11056)

[Sch02] Ralf Schmidt, Some remarks on local newforms for GL(2), J. Ramanujan Math. Soc. 17 (2002), no. 2, 115-147. MR MR1913897 (2003g:11056)

[Ser62] Jean-Pierre Serre, Corps locaux, Publications de l'Institut de Mathématique de l'Université de Nancago, VIII, Actualités Sci. Indust., No. 1296. Hermann, Paris, 1962. MR MR0150130 (27 \#133)

[Ser97]_, Répartition asymptotique des valeurs propres de l'opérateur de Hecke $T_{p}$, J. Amer. Math. Soc. 10 (1997), no. 1, 75-102. MR MR1396897 (97h:11048)

[Ven05] Akshay Venkatesh, Sparse equidistribution problems, period bounds, and subconvexity, Preprint, 2005.

[Wal85] Jean-Loup Waldspurger, Sur les valeurs de certaines fonctions L automorphes en leur centre de symétrie, Compositio Math. 54 (1985), no. 2, 173-242. MR MR783511 (87g:11061b) 
[Xue06] Hui Xue, Central values of Rankin L-functions, Int. Math. Res. Not. (2006), Art. ID 26150, 41. MR MR2249999

[Zha01] Shou-Wu Zhang, Gross-Zagier formula for $\mathrm{GL}_{2}$, Asian J. Math. 5 (2001), no. 2, 183-290. MR MR1868935 (2003k:11101) 